\title{
The ANU WiFeS SuperNovA Programme (AWSNAP)
}

Michael J. Childress ${ }^{1,2,3,12}$, Brad E. Tucker ${ }^{1,2}$, Fang Yuan ${ }^{1,2}$, Richard Scalzo ${ }^{1}$, Ashley Ruiter ${ }^{1,2}$, Ivo Seitenzahl ${ }^{1,2}$, Bonnie Zhang ${ }^{1}$, Brian Schmidt ${ }^{1}$, Borja Anguiano ${ }^{4}$, Suryashree Aniyan ${ }^{1}$, Daniel D. R. Bayliss ${ }^{1,5}$, Joao Bento ${ }^{1}$, Michael Bessell ${ }^{1}$, Fuyan Bian ${ }^{1}$, Rebecca Davies ${ }^{1}$, Michael Dopita ${ }^{1}$, Lisa Fogarty ${ }^{6}$, Amelia Fraser-McKelvie ${ }^{7,8}$, Ken Freeman $^{1}$, Rajika Kuruwita $^{1}$, Anne M. Medling ${ }^{1}$, Simon J. Murphy ${ }^{1}$, Simon J. Murphy ${ }^{6,9}$, Matthew Owers ${ }^{4,10}$, Fiona Panther ${ }^{1,2}$, Sarah M. Sweet ${ }^{1}$, Adam D. Thomas ${ }^{1}$ and George Zhou ${ }^{11,1}$

${ }^{1}$ Research School of Astronomy and Astrophysics, Australian National University, Canberra, ACT 2611, Australia

${ }^{2}$ ARC Centre of Excellence for All-sky Astrophysics (CAASTRO), Canberra, ACT, Australia

${ }^{3}$ School of Physics and Astronomy, University of Southampton, Southampton, SO17 1BJ, UK

${ }^{4}$ Department of Physics and Astronomy, Macquarie University, NSW 2109, Australia

${ }^{5}$ Observatoire Astronomique de l'Université de Genève, 51 ch. des Maillettes, 1290 Versoix, Switzerland

${ }^{6}$ Sydney Institute for Astronomy (SIfA), School of Physics, The University of Sydney, NSW 2006, Australia

${ }^{7}$ School of Physics and Astronomy, Monash University, Clayton, Victoria 3800, Australia

${ }^{8}$ Monash Centre for Astrophysics (MoCA), Monash University, Clayton, Victoria 3800, Australia

${ }^{9}$ Stellar Astrophysics Centre, Department of Physics and Astronomy, Aarhus University, 8000 Aarhus C, Denmark

${ }^{10}$ Australian Astronomical Observatory, PO Box 915, North Ryde, NSW 1670, Australia

${ }^{11}$ Harvard-Smithsonian Center for Astrophysics, 60 Garden St, Cambridge, MA 02138, USA

${ }^{12}$ Email: m.j.childress@ soton.ac.uk

(RECEIVED July 11, 2016; ACCEPTED October 5, 2016)

\begin{abstract}
This paper presents the first major data release and survey description for the ANU WiFeS SuperNovA Programme. ANU WiFeS SuperNovA Programme is an ongoing supernova spectroscopy campaign utilising the Wide Field Spectrograph on the Australian National University 2.3-m telescope. The first and primary data release of this programme (AWSNAP-DR1) releases 357 spectra of 175 unique objects collected over 82 equivalent full nights of observing from 2012 July to 2015 August. These spectra have been made publicly available via the WISEREP supernova spectroscopy repository.

We analyse the ANU WiFeS SuperNovA Programme sample of Type Ia supernova spectra, including measurements of narrow sodium absorption features afforded by the high spectral resolution of the Wide Field Spectrograph instrument. In some cases, we were able to use the integral-field nature of the Wide Field Spectrograph instrument to measure the rotation velocity of the $\mathrm{SN}$ host galaxy near the SN location in order to obtain precision sodium absorption velocities. We also present an extensive time series of SN 2012dn, including a near-nebular spectrum which both confirms its 'super-Chandrasekhar' status and enables measurement of the sub-solar host metallicity at the SN site.
\end{abstract}

Keywords: supernovae: general - supernovae: individual: (SN 2012dn)

\section{INTRODUCTION}

In the last decade, wide-field extragalactic transient surveys-such as the Palomar Transient Factory (PTF; Rau et al. 2009; Law et al. 2009), the Panoramic Survey Telescope and Rapid Response System (PanSTARRS; Kaiser et al. 2010), the Catalina Real-time Transient Survey (CRTS; Drake et al. 2009), the Texas Supernova Search (Quimby 2006; Yuan 2010), and the All-Sky Automated Survey for Supernovae (ASAS-SN; Shappee et al. 2014; Holoien et al. 2016) — have revolutionised our understanding of the myriad ways in which stars explode through the discovery of new classes of exotic transients. Simultane- ously, these surveys have discovered hundreds of supernovae (SNe) of 'traditional' types (see Filippenko 1997, for a review), enabling statistical analyses of the properties of these SNe.

Whilst imaging surveys have provided discovery and light curves for this wealth of new transients, complementary spectroscopy surveys have provided the critical insight into the physical origins of these events. Numerous supernova spectroscopy surveys have released thousands of high-quality spectra of nearby $\mathrm{SNe}$ into the public domain (Matheson et al. 2008; Blondin et al. 2012; Silverman et al. 2012c; Folatelli et al. 2013; Modjaz et al. 2014). These surveys have frequently been dedicated to the spectroscopic follow-up of 
Type Ia supernovae (SNe Ia) which, due to their rates and luminosities, dominate any magnitude-limited imaging survey. Such surveys have revealed that photometrically similar $\mathrm{SNe}$ can still exhibit diversity of spectroscopic behaviour, indicating spectra remain a critical tool for revealing the full nature of the supernova progenitors (particularly for SNe Ia). Additionally, spectra remain critical for supernova classificationparticularly at early phases when the full photometric evolution has yet to be revealed. Such early classifications then inform the use of additional SN follow-up facilities, including those operating outside the optical window.

Recently, the Public ESO Spectroscopic Survey for Transient Objects (PESSTO; Smartt et al. 2015) began a multiyear programme on the NTT 3.6-m telescope in Chile, with the goal of obtaining high-quality spectral time series for roughly 100 transients (of all kinds) to be released to the public. This survey has already released hundreds of spectra in its first two annual data releases, and continues to release all $\mathrm{SN}$ classification spectra within typically $1 \mathrm{~d}$ from observation. Other ongoing SN spectroscopy programmes, such as the Asiago Supernova Programme (Tomasella et al. 2014), also make important contributions to the transient community through timely SN classification and spectroscopy releases.

Here, we describe our ongoing spectroscopy programme AWSNAP - the ANU WiFeS SuperNovA Programmewhich uses the Wide Field Spectrograph (WiFeS; Dopita et al. 2007, 2010) on the Australian National University (ANU) 2.3-m telescope at Siding Spring Observatory in Australia. In this paper, we describe the data processing procedures for this ongoing programme, and describe the first AWSNAP data release (AWSNAP DR1) comprising 357 spectra of 175 supernova of various types obtained during 82 classically scheduled observing nights over a 3-yr period from 2012 July to 2015 August. Most of these spectra have been released publicly via the Weizmann Interactive Supernova data REPository (WISeREP ${ }^{1}$ - Yaron \& Gal-Yam 2012), with the remainder set to be released within the next year as part of forthcoming PESSTO papers. This programme will continue to observe $\mathrm{SNe}$ of interest and classify $\mathrm{SN}$ discoveries from transient searches such as the new SkyMapper Transients Survey (Keller et al. 2007). We aim to release future SN classification spectra from AWSNAP publicly via WISeREP in parallel with any classification announcements.

This paper is organised as follows. Section 2 describes the WiFeS data processing and SN spectrum extraction procedures. Section 3 presents general properties of our SN sample and compares AWSNAP DR1 to other public SN spectra releases. In Section 4, we present some analysis of the properties of the SNe Ia in our sample, including measurement of narrow sodium absorption features afforded by the intermediate resolution of the $\mathrm{WiFeS}$ spectrograph. Some concluding remarks follow in Section 5.

\footnotetext{
${ }^{1}$ http://wiserep.weizmann.ac.il
}

PASA, 33, e055 (2016)

doi:10.1017/pasa.2016.47
Table 1. Details of WiFeS gratings.

\begin{tabular}{ccccc}
\hline \hline Grating & $\lambda_{\min }$ & $\lambda_{\max }$ & Pixel size & Resolution \\
\hline B3000 & $3500 \AA$ & $5700 \AA$ & $0.77 \AA$ & $1.5 \AA$ \\
R3000 & $5400 \AA$ & $9570 \AA$ & $1.25 \AA$ & $2.5 \AA$ \\
R7000 & $5400 \AA$ & $7020 \AA$ & $0.44 \AA$ & $0.9 \AA$ \\
\hline \hline
\end{tabular}

\section{OBSERVATIONS AND DATA DESCRIPTION}

Observations for AWSNAP were conducted with the WiFeS-(Dopita et al. 2007, 2010) on the ANU 2.3-m telescope at Siding Spring Observatory in northern New South Wales, Australia. Observing nights were classically scheduled with a single night of observing every $8-15 \mathrm{~d}$. On some occasions, special objects of interest were observed during non-AWSNAP nights. A full list of the AWSNAP transient spectra is presented in Table A3 in Appendix A. In the sections that follow, we describe the processing of the WiFeS data, then characterise both the long-term performance of the WiFeS instrument and observing conditions at Siding Spring.

\subsection{Data reduction and supernova spectrum extraction}

The WiFeS instrument is an image-slicing integral field spectrograph with a wide $25 \operatorname{arcsec} \times 38$ "arcsec field of view. For AWSNAP, this frequently provided simultaneous integral field observations of $\mathrm{SNe}$ and their host galaxies. The WiFeS image slicer breaks the field of view into 25 'slitlets' of width 1 arcsec, which then pass through a dichroic beamsplitter and volume phase holographic (VPH) gratings before arriving at $4 \mathrm{k} \times 4 \mathrm{k}$ CCD detectors. AWSNAP observations were always conducted with a CCD binning of 2 in the vertical direction-this sets the vertical spatial scale of the detector to be 1 arcsec, yielding final integral field elements (or 'spaxels') of size 1 arcsec $\times 1$ arcsec. Typically seeing at Siding Spring is roughly $2 \operatorname{arcsec}$ (see Section 2.3).

The VPH gratings utilised by WiFeS provide a higher wavelength resolution than traditional glass gratings. The low- and high-resolution gratings provide resolutions of $R=$ 3000 and $R=7000$, respectively, yielding velocity resolutions of up to $\sigma_{\mathrm{v}} \sim 45 \mathrm{~km} \mathrm{~s}^{-1}$ which is ideal for observing nebular emission lines from ionised regions in galaxies. For $\mathrm{SNe}$, this can reveal narrow absorption features (see Section 4.2) from circumstellar material (CSM) which are typically smeared out by lower resolution spectrographs. AWSNAP observations were generally conducted with the lower resolution B3000 and R3000 gratings for the blue and red arms of the spectrograph, respectively, with the RT560 dichroic beamsplitter. Occasionally, the R7000 grating was deployed on the red arm to provide higher resolution observations of sodium absorption features. In Table 1, we provide the wavelength range, spectroscopic pixel size, and wavelength resolution (determined as the FWHM of calibration 
lamp emission lines) for the three gratings used for AWSNAP observations.

Data for AWSNAP observations were reduced with version 0.7.0 of the PyWiFeS pipeline (Childress et al. 2014a). PyWiFeS performs standard image pre-processing such as overscan and bias subtraction, as well as cosmic ray rejection using a version of LACosmic (van Dokkum 2001) tailored for WiFeS data. The wavelength solution for WiFeS is derived using an optical model of the spectrograph which achieves an accuracy of 0.05 to $0.10 \AA$ (for $R=7000$ and $R=3000$, respectively) across the entire detector. Spectral flatfielding (i.e. correction of pixel-to-pixel quantum efficiency variations) is achieved with an internal quartz lamp, whilst spatial flat-fielding across the full instrument field of view is facilitated by twilight sky flats. Once the data has been preprocessed and flat-fielded, it is resampled onto a rectilinear three-dimensional $(x, y, \lambda)$ grid (a 'data cube'). We then flux calibrate the data cubes with the use of spectrophotometric standard stars (from, e.g. Oke 1990; Bessell 1999; Stritzinger et al. 2005) and the Siding Spring Observatory extinction curve from Bessell (1999), whilst telluric features are removed using observations of smooth spectrum stars.

To further facilitate data reduction for the AWSNAP data release, we developed an SQL database for WiFeS observations using the Python Django framework. We created modified versions of the PyWiFeS reduction scripts that allow the user to request that all data from a specific night using a specific grating be fully reduced. The reduction scripts then query the database for all science observations and calibration data from that night and perform the required reduction procedures. For nights where a full suite of calibrations was not available (e.g. due to guest observations on a nonAWSNAP night), calibration solutions from the closest (in time) AWSNAP night were automatically identified via the database and employed in the reduction. Both the wavelength solution and spatial flat-fielding of the instrument are incredibly stable on long ( year) timescales (see Section 2.2), thus validating the choice to use calibrations from different nights where necessary. This new database-driven data processing mode for PyWiFeS will be an important component of the upcoming effort to develop fully robotic queue-based observing capabilities for the ANU 2.3-m telescope.

The output of the PyWiFeS pipeline is a flux calibrated data cube that contains signal from the target supernova, the night sky, and occasionally the SN host galaxy. Extraction of the SN spectrum requires isolation of the spaxels containing supernova signal and subtraction of the underlying sky (and possibly galaxy) background. To achieve this, we constructed a custom Python-based GUI which allows the user to manually select spaxels containing the target SN ('object' spaxels) and spaxels used to determine the background ('sky' spaxels, which may contain some galaxy signal). The background spectrum is determined as the median spectrum across all 'sky' spaxels, and this median spectrum is subtracted from each 'object' spaxel. The final SN spectrum is then the sum of the sky-subtracted object spaxels, and the variance spectrum is the sum of the variance from all object spaxels (with no subtraction of sky variance).

This SN spectrum extraction technique produces excellent quality sky subtraction due to the robust spatial flat-fielding achieved with PyWiFeS. However, some obvious sky subtraction residual features are evident in the redder wavelengths of most R3000 spectra where the night sky exhibits sharp emission features from rotational transitions of atmospheric $\mathrm{OH}$ molecules. The intrinsic line width of these emission lines is below the resolution of the spectrograph, meaning the observed line width is that of the spectrograph-which in this case is only slightly larger than the detector pixel size. The natural wavelength solution of the spectrograph shifts spaxelto-spaxel, so when all spaxels are resampled onto the same wavelength grid this means sky lines experience pixel-wise resampling that is not uniform across the full instrument field of view. Thus, when a median sky spectrum is subtracted from a specific spaxel, some residual features arise due to this resampling effect. A more robust technique for correcting this would be to model the intrinsic sky spectrum using the multiple samplings achieved across all spaxels and resample it to the wavelength solution of each spaxel before subtracting it (as was demonstrated for two-dimensional spectroscopic data by Kelson 2003). Such a technique is beyond the scope of the current data release, but is being prioritised for future AWSNAP releases.

\subsection{Long-term behaviour of the WiFeS instrument}

One key advantage of having a long-running observing programme is the ability to characterise the long-term behaviour of the WiFeS instrument. Below we analyse the stability of the wavelength solution and instrument throughput on multiyear timescales. WiFeS did undergo a major change in early 2013 when the detectors for both arms of the spectrograph were replaced with higher throughput E2V CCDs. Thus, we restrict our analysis to dates from late 2013 May until the end of the current data release in 2015 August.

We collected the wavelength solution fits for the B3000 (R3000) grating from 48 (45) distinct epochs following the WiFeS detector upgrade. In Figure 1, we plot the difference between the wavelength solution for each individual epoch and the mean wavelength solution across all epochs. Epochs are colour-coded from earliest (red) to latest (purple) to illustrate potential coherent long-term shifts in the wavelength solution. The top panels present the B3000 grating, whilst the bottom panels present the R3000 gratings. The left and middle columns represent the WiFeS slitlets at the top and middle of the detectors, respectively. In the right hand panels of this Figure, we show the average deviation of the wavelength solution for a given epoch from the global mean wavelength solution, as a function of epoch. This allows us to track any coherent evolution of the detector wavelength solution with time.

From these plots, we see clear demonstration of the remarkable stability of the WiFeS instrument. The RMS 

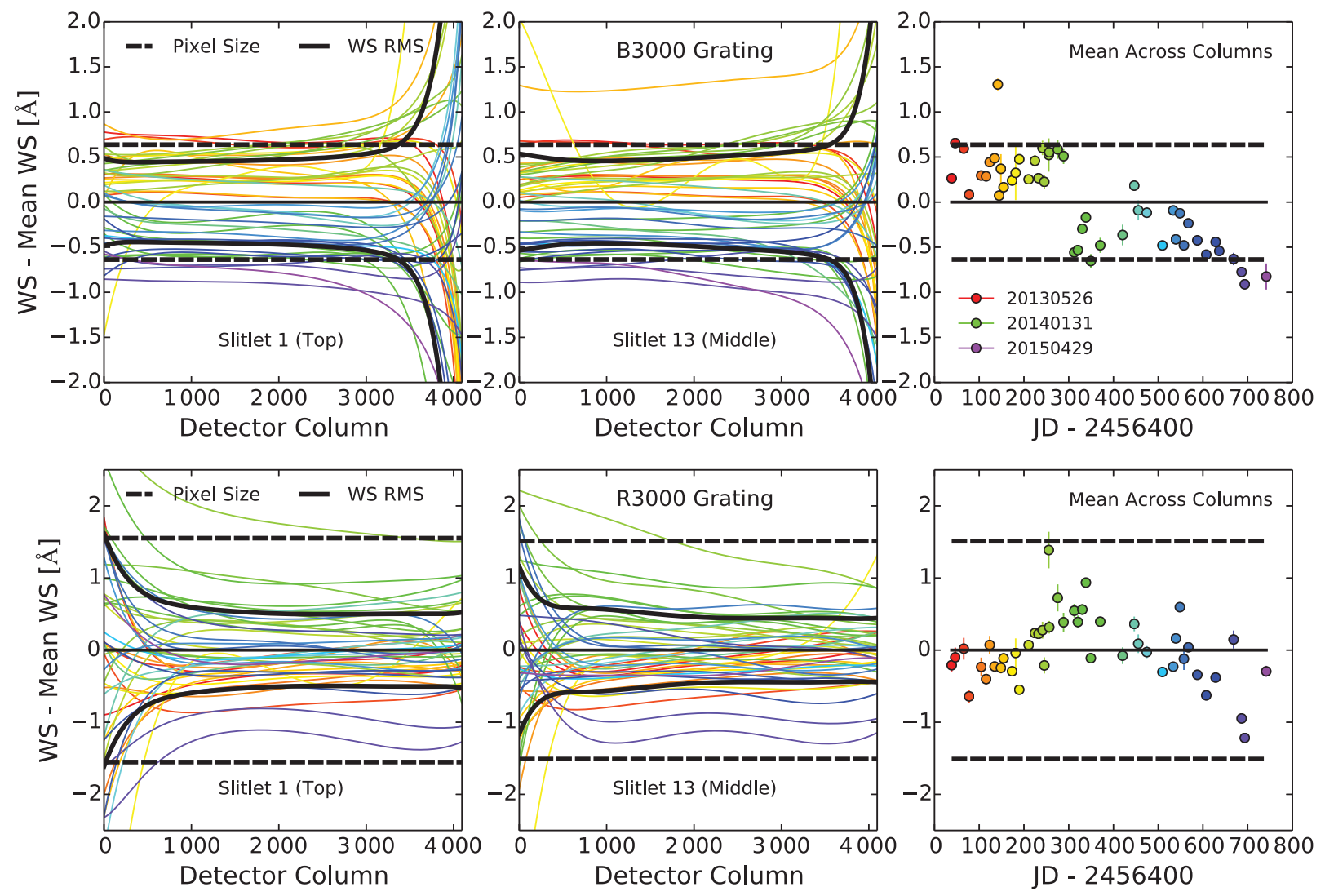

Figure 1. Evolution of the WiFeS wavelength solution over a 2-yr period spanning 2013 May to 2015 April. In the left two panels of each row, we plot the deviation of individual wavelength solutions from the mean solution (averaged over the full 2-yr period) for the top and middle slitlets of the instrument (left and middle columns, respectively) for the B3000 (top row) and R3000 (bottom row) gratings. Wavelength solution residuals are colour-coded by date from earliest (red) to latest (purple), with the pixel size (dashed black lines) and wavelength solution residual RMS (solid black lines) displayed for comparison. In the right panels of each row, we show the average deviation (across all wavelengths) from the mean wavelength solution as a function of epoch, to trace the temporal evolution of the instrument solution (points for individual epochs obey the same colour scheme as the left panels).

variation of wavelength solution is smaller than a single pixel for both gratings (i.e. both detectors) for nearly all wavelengths, with the exception of the lower throughput regions near the dichroic boundary. There is some evidence of a coherent shift of the blue detector wavelength solution over the 2-yr time period probed here, but this is still less than two pixels shift. For the red detector, we can say confidently that the wavelength solution has shifted by less than a pixel over a timescale of 2 yrs. This remarkable stability achieved (by design) by WiFeS means the use of the wavelength solution from an adjacent night yields negligible changes in wavelength, and thus small instrumental velocity shifts-highly suitable for supernova analyses.

We then collected illumination corrections (derived from twilight flat observations) from 46 (45) distinct epochs for the B3000 (R3000) grating. In Figure 2, we show the mean illumination correction (left panels) and RMS of the illumination correction (right panels- presented in fractional form, i.e. the RMS divided by the mean) for both the B3000 (top) and R3000 (bottom) gratings. We find the mean variation of the illumination correction to be 1.4 and $1.2 \%$ for the $\mathrm{B} 3000$ and R3000 gratings, respectively, for the full WiFeS field of view. The nature of the instrument optics are such that the outer regions of instrument field of view have slightly lower throughput, and thus a higher fractional RMS than the inner most region. For the innermost 8 arcsec $\times 8$ arcsec region typically used for SN observation in AWSNAP, the RMS of the illumination correction is 0.8 and $0.3 \%$ for the blue and red detectors (i.e. B3000 and R3000 gratings). Thus, the throughput of the WiFeS instrument has remarkable spatial uniformity on long (multi-year) timescales.

\subsection{Observing conditions at siding spring observatory}

In addition to the long-term behaviour of the WiFeS instrument, our observing programme allows us to monitor the observing conditions at Siding Spring Observatory. Below we briefly discuss the atmospheric throughput and seeing conditions experienced during AWSNAP observing.

We collected the flux calibration solutions from 59(54) epochs for the B3000 (R3000) grating from 2013 May to 

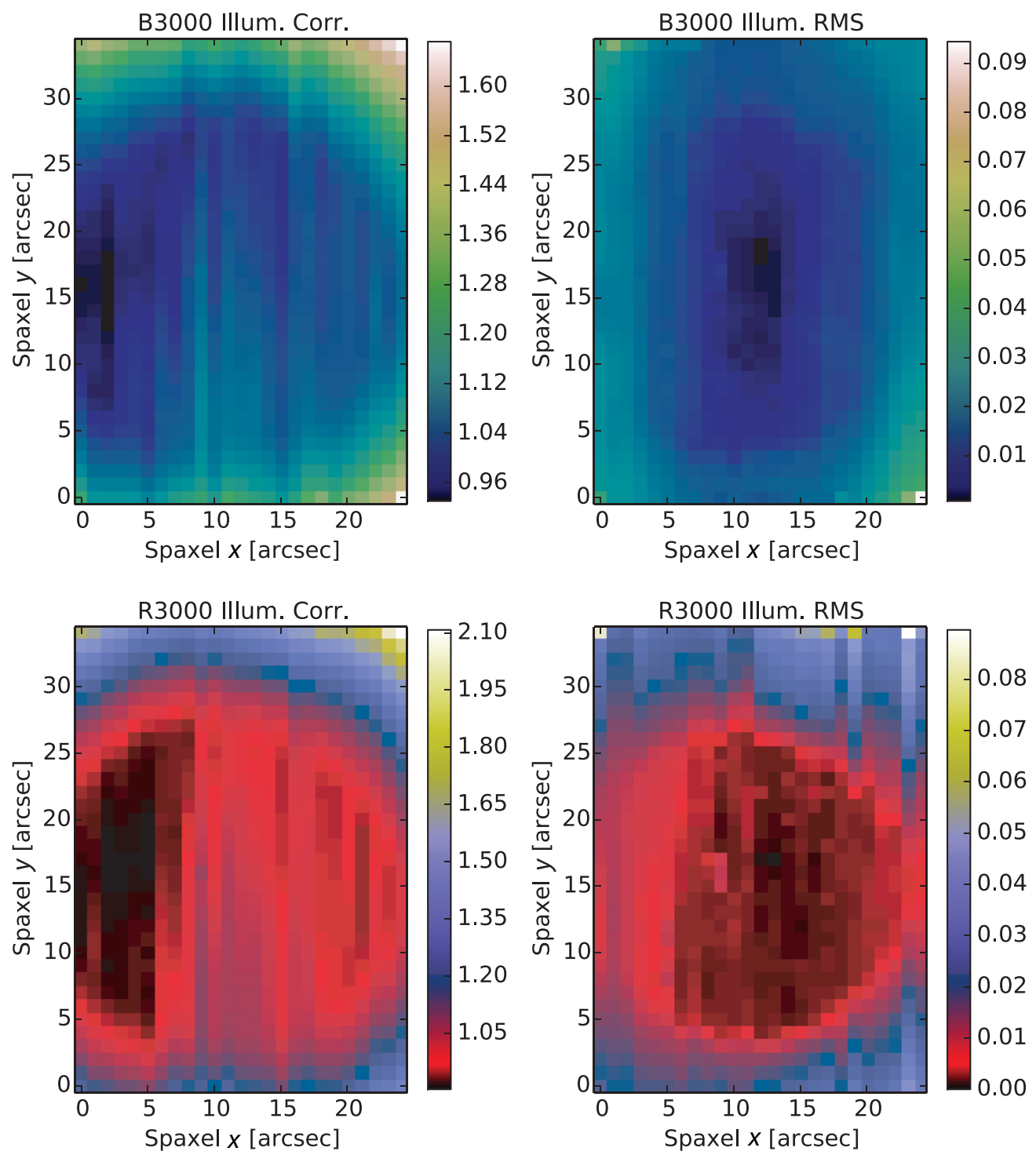

Figure 2. The normalised mean (left column) and RMS (right column) of the illumination corrections for the B3000 (top row) and R3000 (bottom row) gratings. The RMS images are shown as fractional values of the mean illumination correction.

2015 August, and these are plotted in Figure 3 after being normalised in the wavelength range with highest throughput (45 00-5 $400 \AA$ and $6500-8000 \AA$ for B3000 and R3000, respectively). These curves represent the normalised throughput (in magnitudes) of the instrument and atmosphere as measured with spectrophotometric standard stars (typically from Oke 1990; Bessell 1999; Stritzinger et al. 2005) whose flux has already been corrected using the nominal Siding Spring extinction curve from Bessell (1999). The effects of the instrument throughput and atmospheric transmission are degenerate here, as WiFeS instrument throughput cannot be independently measured using local (i.e. terrestrial) calibration sources.

From the curves in Figure 3, we see that the total combined throughput of the instrument plus atmosphere is relatively stable. We calculated the RMS colour variation in the through- put curves and find variations of $\sigma(U-B)=0.09$ mag for $\mathrm{B} 3000$ (note the $V$ band runs into the wavelength range where the dichroic splits light between the blue and red channels) and $\sigma(r-i)=0.04 \mathrm{mag}$ for R3000. We note these are calculated from the mean flux calibration solution for each night, where no attempt has been made to derive a unique extinction curve for a given night. These colour variations are relatively small, and comparable in size to the colour dispersion found when comparing spectrophotometry to imaging photometry for other SN spectroscopy samples (e.g. Silverman et al. 2012c; Blondin et al. 2012; Modjaz et al. 2014).

We also measured the atmospheric seeing during our observing programme, using both guide star FWHM measurements recorded from the WiFeS guider camera and low airmass $(\sec z \leq 1.5)$ WiFeS data cubes convolved with the $B$ - and $R$-band filter curves (for the blue and red cameras, 

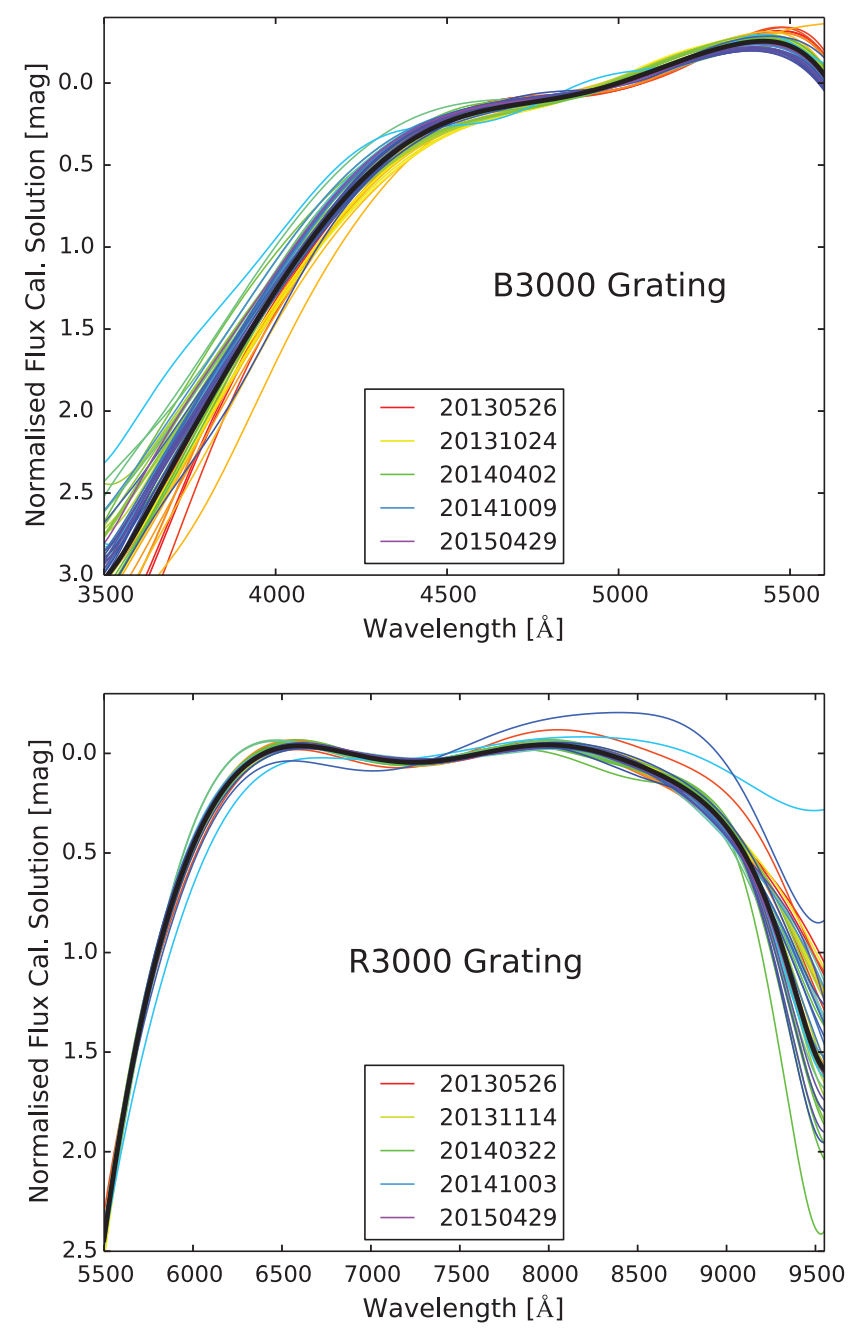

Figure 3. Flux calibration solutions for the B3000 (top) and R3000 (bottom) gratings. As in Figure 1, these are colour-coded by date from earliest (red) to latest (purple), with the mean flux calibration solution shown as the solid black line.

respectively). We plot the observed distribution of seeing values in Figure 4. The seeing distribution peaks slightly above 1.5 arcsec (for all measurements) with a tail predominantly filled to 2.5 arcsec, with little or no sub-arcsecond observations and a small number of observations with incredibly poor seeing (3.0 arcsec or greater).

\section{THE AWSNAP SUPERNOVA SAMPLE AND SPECTROSCOPIC DATA RELEASE}

In this Section, we briefly describe the global characteristics of the sample of $\mathrm{SNe}$ comprising the first data release (DR1) for AWSNAP. This consists of observations made between 2012 July 18 and 2015 August17, a total of 357 epochs of 175 total SNe. These spectra have all been uploaded to WISeREP (Yaron \& Gal-Yam 2012), with most made publicly available and the small remainder set to be made public with the associated PESSTO publication within the next year. Some addi-

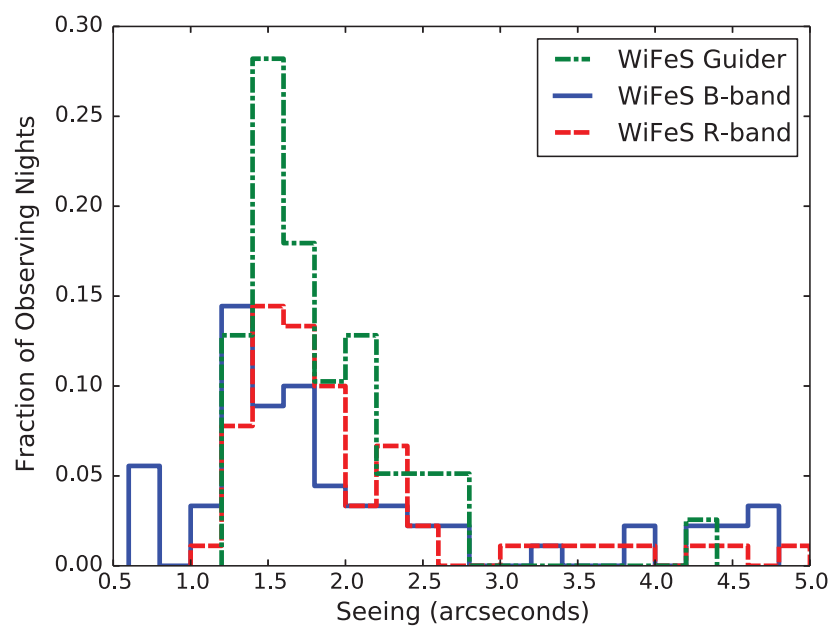

Figure 4. Seeing measurements at Siding Spring Observatory as measured during AWSNAP observations. These include measurements from the WiFeS guider camera (green dash-dot histogram), and measurements of low airmass standard star WiFeS datacubes convolved with $B$-band (blue solid histogram-from the blue detector) and $R$-band (red dashed histogramfrom the red detector) filter curves.

tional spectra taken after 2015 August 17 have been processed and released via WISeREP, and we expect the future release of AWSNAP spectra to proceed in a continuous fashion via the same procedures outlined in this work.

AWSNAP originated as the spectroscopic observation programme for the supernova group at the ANU. The programme was intended to cover a broad range of science topics driven by transient discoveries from contemporaneous photometric surveys, with a large emphasis on timely classification of newly discovered transients. The observing and target selection strategy for AWSNAP was heavily influenced by the scheduling of our observing time, which typically consisted of one single classically scheduled full night of observing every $8-15 \mathrm{~d}$ throughout the entirety of the calendar year. The most significant implication of this scheduling was that dense spectroscopic sampling (i.e. 2-3 d cadence) was generally not viable for our preferred targets (though on rare occasions we requested time exchange with other observers for targets of particular importance). Furthermore, additional targets were always needed to fill an entire night of observing. Thus, we frequently chose to make complementary observations of targets being observed through the PESSTO programme (Smartt et al. 2015), or chose targets whose spectroscopic data would have legacy value for future analyses. As a result, we observed a diverse range of targets that covered the entire Southern sky. The full list of targets and their classification information in presented in Table A1 in Appendix A, and their distribution on the sky is shown in Figure 5.

The AWSNAP spectra have relatively high signal-to-noise (S/N) by design so that they might be useful for precision spectral feature measurement. Our sample's median $\mathrm{S} / \mathrm{N}$ is 16 per $\AA$, with $1 \sigma$ high and low $\mathrm{S} / \mathrm{N}$ values of 40 and 6 per $\AA$, respectively. We show some example AWSNAP spectra in 


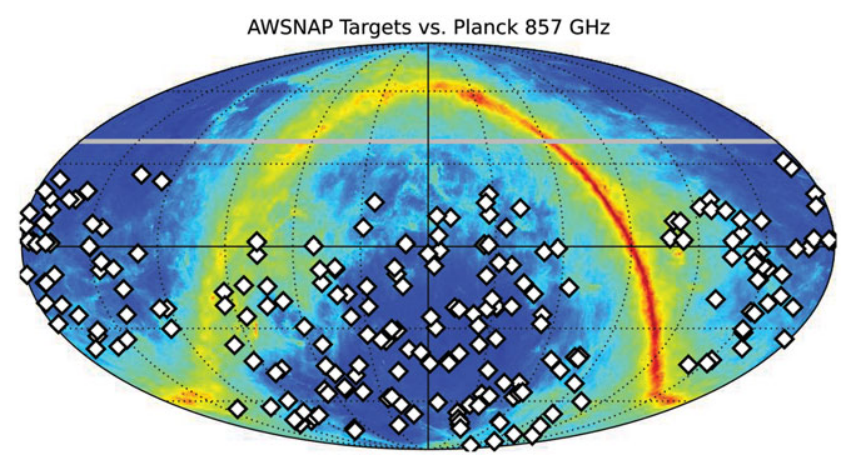

Figure 5. On-sky distribution (in equatorial coordinates) of all extragalactic targets in AWSNAP DR1 (white diamonds) plotted over the $857 \mathrm{GHz}$ allsky map from the Planck satellite (Planck Collaboration et al. 2011) which reveals emission by Milky Way dust. The physical pointing limit of the ANU 2.3-m telescope $\left(\sim+40^{\circ}\right.$ declination $)$ is shown as the solid gray bar.

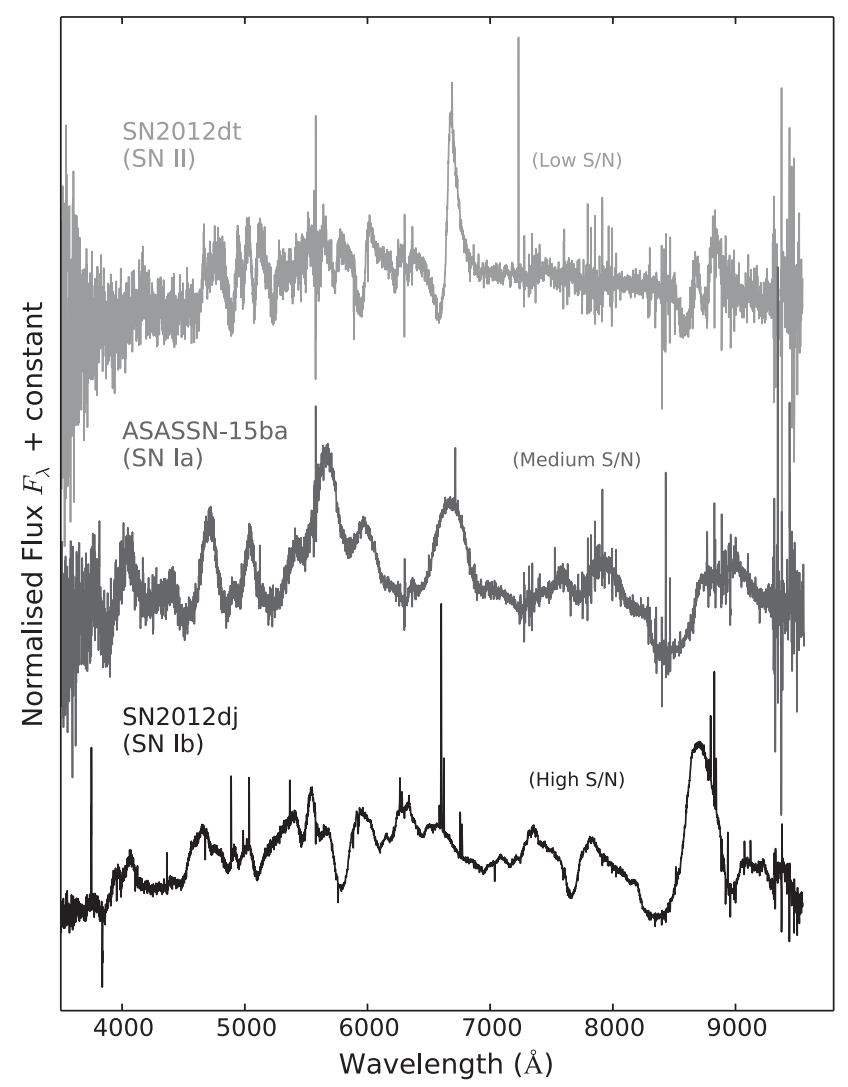

Figure 6. Example spectra spanning the typical range of signal-to-noise $(\mathrm{S} / \mathrm{N})$ in the AWSNAP sample. Shown here are spectra of SN 2012dt (a SN II, with a 'low' S/N of 5 per $\AA$ ), ASASSN-15ba (a SN Ia, with a 'medium' S/N of 13 per $\AA$ ), and SN 2012dj (a SN Ib, with a 'high' S/N of 33 per $\AA$ ).

Figure 6 exhibiting low $(-1 \sigma)$, medium (median), and high $(+1 \sigma) \mathrm{S} / \mathrm{N}$.

In Figure 7, we present a histogram of the number of spectroscopic observations per target for the AWSNAP sample compared to three major SN Ia spectroscopic data releases: the Berkeley SN Ia Programme (BSNIP; Silverman et al. 2012c), the Harvard Center for Astrophysics (CfA) Super-
Table 2. AWSNAP objects and spectra by SN type.

\begin{tabular}{lcc}
\hline \hline SN type & \# Objects & \# Spectra \\
\hline SN Ia & 101 & 180 \\
SN II & 53 & 117 \\
SN Ibc & 15 & 43 \\
SLSN & 6 & 17 \\
Total & 175 & 357 \\
\hline \hline
\end{tabular}

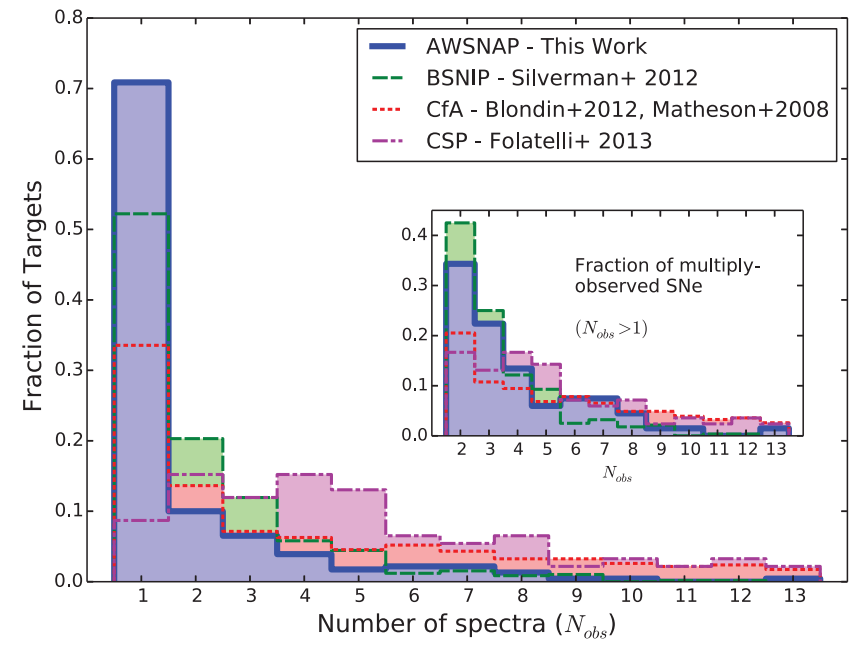

Figure 7. Normalised histogram of the number of spectroscopic epochs per target for the full sample of AWSNAP DR1 targets. For comparison, we also show the same histogram for previous SN spectroscopy surveys: BSNIP (Silverman et al. 2012c), CfA (Matheson et al. 2008; Blondin et al. 2012), and CSP (Folatelli et al. 2013). The inset shows the (re-)normalised histograms of the number of spectroscopic epochs for multiply observed targets (i.e. those with $N_{\mathrm{obs}}>1$ ) in the same surveys.

nova Programme (Matheson et al. 2008; Blondin et al. 2012), and the Carnegie Supernova Project (CSP; Folatelli et al. 2013). Our sample has a high fraction of singly observed targets compared to the other programmes. This is in part due to the fact that our data release includes all our SN classification spectra, as well as the persistent need for us to fill our classically scheduled observing queue. We thus also show in the inset of Figure 7 the normalised histogram of spectroscopic epochs for multiply observed $\mathrm{SNe}$ (i.e. targets with $N_{\mathrm{obs}}>2$ ), which shows a much more similar distribution to the other programmes.

The types of SNe observed in AWSNAP DR1 is summarised in Table 2 and presented graphically in Figure 8. We show both the number of targets and number of spectra for AWSNAP, and for reference we compare this to the magnitude-limited rates for $\mathrm{SNe}$ in the local universe calculated by Li et al. (2011a). For this figure, we have grouped the SNe by type into four main categories:

- SNe Ia: includes the standard (Branch-normal) SNe Ia, sub-luminous (SN 1991bg-like Filippenko et al. 1992a), over-luminous (SN 1991T- and SN 1999aa-like 


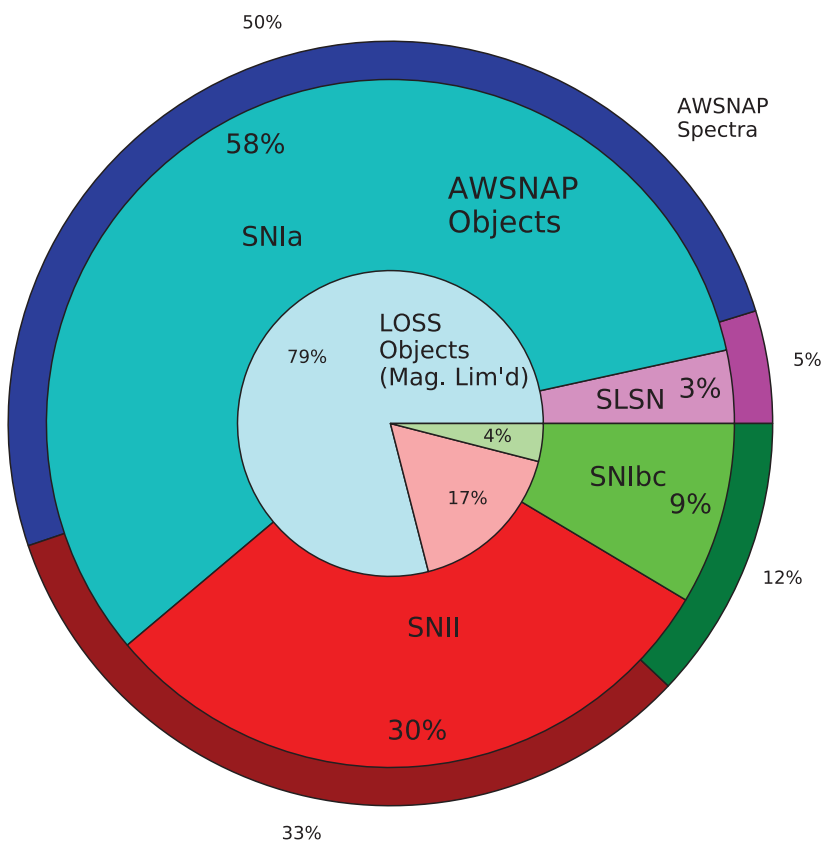

Figure 8. Total number of spectra (outer ring) and SN targets (middle annulus) for AWSNAP broken down by SN type, compared to the volume-limited SN rates (inner circle) for the LOSS survey ( $\mathrm{Li}$ et al. 2011a, -note this galaxy-targeted survey did not find any SLSNe). In each ring, the regions are colour-coded by (broad) SN type (see text for discussion): SNe Ia (blue), $\mathrm{SNe} \mathrm{II}(\mathrm{red}), \mathrm{SNe} \mathrm{Ib} / \mathrm{Ic}$ (green), and SLSNe (purple).

Filippenko et al. 1992b), candidate 'superChandrasekhar' SNe Ia (Scalzo et al. 2010, 2012), and SNe Iax (Foley et al. 2013).

- SNe Ibc: SNe Ic, SNe Ib, and SNe IIb-the standard classes of 'stripped-envelope' SNe (e.g. Bianco et al. 2014; Modjaz et al. 2014; Graur et al. 2015; Liu et al. 2016).

- SNe II: SNe IIP and SNe IIL—which we note cannot be distinguished spectroscopically — and SNe IIn.

- SLSNe: 'superluminous' SNe-for our sample, this consists entirely of the 'SLSN-Ic' type (see, e.g. Inserra et al. 2013) which show blue continua with weak absorption features and no hydrogen signatures.

We note these groupings are made strictly to provide broad perspective on the sample statistics, but we reiterate that the SN sub-types within each group have their own unique physical mechanisms.

From Figure 8, we clearly see that, as expected, SNe Ia comprise the majority of objects and spectra in the AWSNAP sample, but comprise a smaller fraction than might be expected from a pure magnitude limited sample of SNe such as that of the Lick Observatory Supernova Search (LOSS, whose relative $\mathrm{SN}$ rates are presented in $\mathrm{Li}$ et al. 2011a). This may be due in part to the fact that many of the SNe in AWSNAP were discovered by untargeted supernova searches such as the ASAS-SN (Shappee et al. 2014). These surveys find SNe in low-mass galaxies that are missed by targeted surveys such

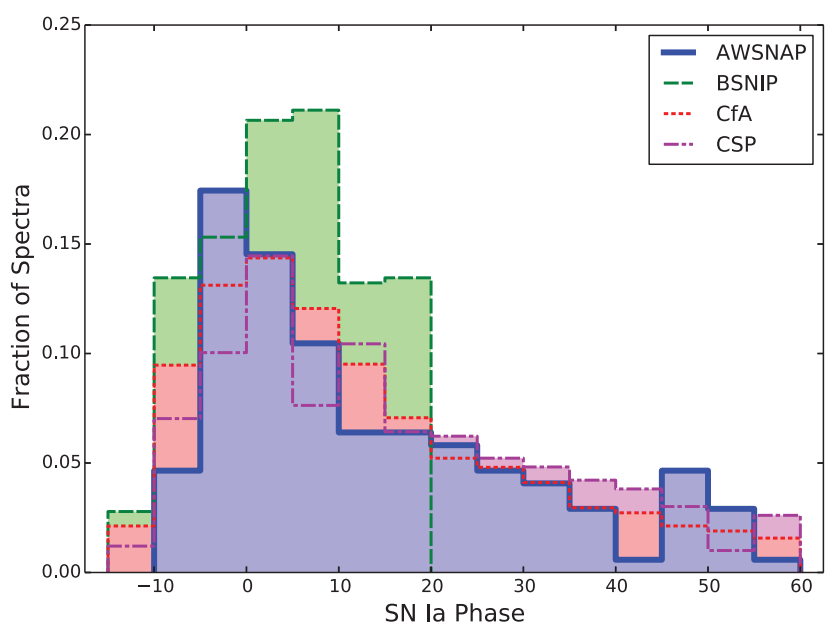

Figure 9. Histograms of spectroscopic phases (with respect to $B$-band maximum light) for SN Ia spectra in AWSNAP and other SN Ia spectroscopy samples (the same as in Figure 7). Note the AWSNAP phases are based on the spectroscopic-based phase reported with the SN classification, which may have an associated uncertainty of 3-5 d.

as LOSS. Due to the increased star-formation intensity in low-mass galaxies (e.g. Salim et al. 2007), this means the relative rate of core-collapse $\mathrm{SNe}$ will be higher and thus CCSNe will comprise a higher fraction of the sample. Additionally, we explicitly targeted a higher fraction of CCSN discoveries owing to the comparative paucity of CCSN spectroscopic samples compared to SNe Ia (again arising from the magnitude-limited rates). Thus, our higher representation of CCSNe than the expectation from the magnitude-limited rates of Li et al. (2011a) is likely a combination of the higher CCSN fraction for our untargeted feeder surveys and our explicit emphasis on preferential targeting of CCSNe.

\section{TYPE IA SUPERNOVA SPECTRA FROM AWSNAP}

As a first demonstration of the usefulness of the AWSNAP dataset, we analyse basic spectroscopic features of the SNe Ia in our sample-this comprises 180 total spectra of $101 \mathrm{ob}-$ jects. In Figure 9, we plot a histogram of the phases ${ }^{2}$ of our SN Ia spectra, again compared to previous large SN Ia spectroscopy surveys (CfA, BSNIP, CSP). Our sample shows a similar phase coverage as previous surveys, with perhaps a slight increase in earlier phases due to improved SN discovery efficiency from nearby SN searches. In the sections that follow, we further analyse features of interest from the AWSNAP SN Ia spectroscopy sample.

\subsection{Spectral features in SNe Ia at maximum light}

We begin by inspecting the spectra of SNe Ia close to the epoch of peak brightness. These maximum light spectra have

\footnotetext{
${ }^{2}$ Based on the spectroscopically estimated phase reported at the time of classification.
} 
a long history of providing key insights into the diversity of SN Ia explosions through the study of 'spectral indicators' (Nugent et al. 1995; Hatano et al. 2000; Benetti et al. 2005; Bongard et al. 2006, 2008; Hachinger, Mazzali, \& Benetti 2006; Hachinger et al. 2008; Branch et al. 2006; Branch, Dang, \& Baron 2009; Bronder et al. 2008; Silverman, Kong, \& Filippenko 2012b), as well as potential avenues for improving the cosmological standardisation of SNe Ia (Wang et al. 2009; Bailey et al. 2009; Blondin, Mandel, \& Kirshner 2011; Silverman et al. 2012a). Thus, we actively targeted many $\mathrm{SNe}$ Ia (which had already been classified) on an epoch close to maximum light to obtain high-quality spectra facilitating such studies.

We isolated the sample SNe Ia with a spectrum within $5 \mathrm{~d}$ of estimated peak brightness. It is important to reiterate here that the phases for our SN Ia sample are extrapolated from the reported spectroscopic classification phase and date. It has been demonstrated previously (e.g. Blondin et al. 2012) that the phase determined for $\mathrm{SNe}$ Ia via spectroscopic matching codes such as SNID (Blondin \& Tonry 2007) typically has an uncertainty of at least 3-5 d. Thus, we may have included SN Ia spectra as much as $10 \mathrm{~d}$ removed from maximum light. Our analysis here is intended to be illustrative of the utility of our published spectra, and more detailed quantitative spectroscopic analyses should always be coupled to a robust photometric data set.

For the analysis that follows, we measure two key quantities of interest: the silicon absorption ratio $R_{\mathrm{Si}}$, and the strength of high-velocity features (HVFs) $R_{\mathrm{HVF}}$. For this work, we define $R_{\mathrm{Si}}$ as the ratio of the pseudo equivalent width (pEW) of the $5962 \AA$ feature to the pEW of the $6355 \AA$ feature. The $\mathrm{pEW}$ for each feature is measured by fitting a linear pseudo-continuum across narrow regions redward and blueward of the given feature (similar to methods employed in Silverman et al. 2012b), then integrating the flux in the normalised absorption feature.

$R_{\mathrm{Si}}$ is known to correlate strongly with the $\mathrm{SN}$ Ia light curve decline rate (Nugent et al. 1995). We quantified the relationship between our definition of $R_{\mathrm{Si}}$ (the $\mathrm{pEW}$ ratio) and light curve decline rate $\Delta m_{15}$ by fitting a linear relationship between these quantities for a sample of $342 \mathrm{SNe}$ Ia collated from the CfA, BSNIP, and CSP samples. The fit to these data is presented in Appendix A2, shown graphically in Figure A1 with a best fit trend given by Equation (A1). Below we will use this relation to display the corresponding range of $\Delta m_{15}$ spanned by our observed values of $R_{\mathrm{Si}}$.

We measure the HVF strength for our SN Ia sample using the same techniques employed in Childress et al. (2014b), and similarly define $R_{\mathrm{HVF}}$ as the ratio of the $\mathrm{pEW}$ of the HVF to the pEW of the photospheric velocity feature (PVF). Briefly, we first normalise the Ca NIR feature using a linear pseudo-continuum fit. We then fit the Ca NIR feature with two Gaussians in velocity space with velocity centres, widths, and absorption depths fitted with a Python mpfit routine. Each component in velocity space corresponds to a triplet in wavelength space, and we set the absorption depth of each

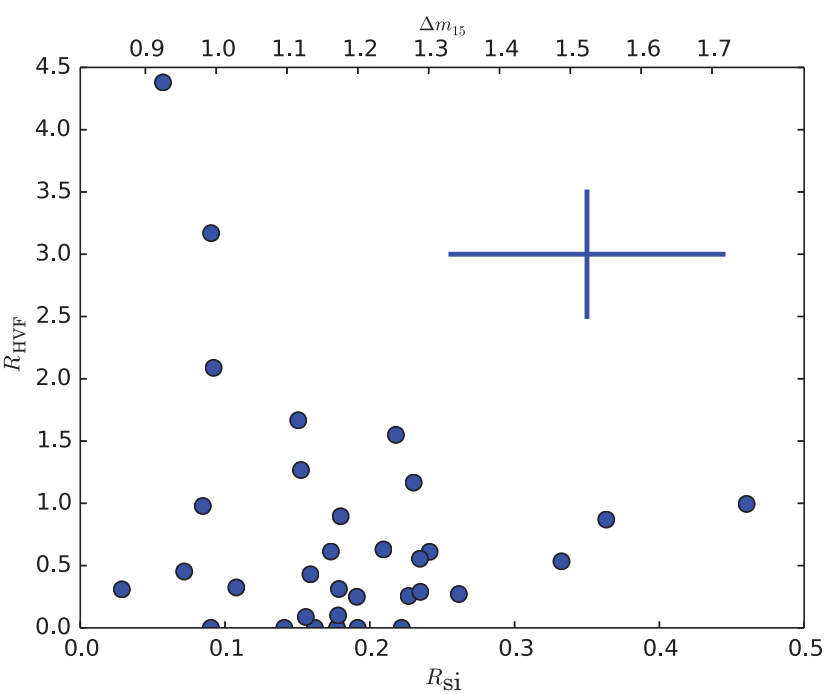

Figure 10. Strength of the high-velocity features (HVFs) in the Ca II NIR triplet-using the quantity $R_{\mathrm{HVF}}$ as defined by Childress et al. (2014b) plotted against the $\mathrm{Si}$ II absorption strength ratio $R_{\mathrm{Si}}$ defined by Nugent et al. (1995). On the top axis, we show the rough equivalent light curve decline rate $\Delta m_{15}$ values corresponding to the range of $R_{\mathrm{Si}}$ values. The crosshair in the upper right represents the characteristic errors in measurement of $R_{\mathrm{HVF}}$ (typically $20 \%$, plotted here for the larger values of $R_{\mathrm{HVF}}$ ) and the error in $\Delta m_{15}$ when converted from $R_{\mathrm{Si}}$.

component equal to each other (i.e. the optically thick limit). As in Childress et al. (2014b), we require the photospheric velocity component to have a velocity centre within $20 \%$ of the value derived for the $\mathrm{Si} 6355 \AA$ feature, though in most cases the results are the same if this requirement is removed.

In Figure 10, we plot the measured HVF strength $\left(R_{\mathrm{HVF}}\right)$ versus the silicon absorption strength ratio $R_{\mathrm{Si}}$. For illustrative purposes, we also show the scale of light curve decline rate $\Delta m_{15}$ corresponding to the plotted range of $R_{\mathrm{Si}}$ using the above relation. A representative error bar for $\Delta m_{15}$ from the above relation and a typical $R_{\mathrm{HVF}}$ error-bar are shown in the figure.

Figure 10 demonstrates a tendency for SNe Ia with strong HVFs to also have low values of $R_{\mathrm{Si}}$ and thus broad light curves (low $\Delta m_{15}$ ). This correlation of HVFs with SN Ia light curve width was first observed by Maguire et al. (2012) in composite high-redshift SN Ia spectra, and subsequently confirmed by numerous studies of low-redshift SNe Ia (Childress et al. 2014b; Maguire et al. 2014; Pan et al. 2015; Silverman et al. 2015; Zhao et al. 2015). Our results support these studies with spectra alone.

\subsection{Narrow sodium absorption features in SN Ia spectra}

A great advantage of the higher resolution of WiFeS (compared to many spectrographs deployed for other SN spectroscopy surveys) is the ability to detect narrow absorption features, particularly the Na I doublet at $\lambda \lambda 5890 / 5896 \AA$. This feature has a long history of being used to infer the 
presence of foreground dust in SNe Ia (Barbon et al. 1990; Turatto, Benetti, \& Cappellaro 2003; Poznanski et al. 2011; Poznanski, Prochaska, \& Bloom 2012; Phillips et al. 2013). Earlier works attempted to derive correlations between SN Ia colours and sodium absorption strength (i.e. the absorption equivalent width), but Poznanski et al. (2011) showed sodium to be a poor indicator of SN Ia reddening. Phillips et al. (2013) refined this result by showing that SN Ia reddening exhibits a strong correlation with absorption strength of the diffuse interstellar bands (found exclusively in the interstellar medium) but some SNe Ia have an excess of sodium absorption that does not coincide with associated reddening of the SN. Thus, sodium features in SNe Ia remain instructive but should be considered with a measure of caution.

Recently, velocities of sodium absorption features in SN Ia spectra have been used as a diagnostic of CSM, including cases of some SNe Ia (Patat et al. 2007; Simon et al. 2009) where the sodium absorption features exhibit variability (though most do not-see Sternberg et al. 2014), indicating SN-CSM interaction. More recently, a statistical analysis of the velocity distribution of sodium features in SNe Ia by Sternberg et al. (2011) found an excess of SNe Ia with blueshifted sodium features, indicating that a fraction of SNe Ia explode inside an expanding shell of material that was presumably shed by the SN Ia progenitor system prior to explosion. Maguire et al. (2013) extended this work to show that the excess of SNe Ia with blueshifted sodium absorption was populated predominantly by the more luminous SNe Ia with slow declining light curves (i.e. high 'stretch').

We thus searched for the presence of sodium absorption features in our sample of SN Ia spectra. For most SNe Ia (13), this was done with the lower resolution (R3000) observations of the SN at maximum light. For six SNe Ia, we also had a higher resolution (R7000) observation of the $\mathrm{SN}$ at maximum light. In a few instances, this observation was triggered by the presence of strong reddening being reported in the SN classification announcement. For the other instances, we obtained both a low-resolution (R3000) and high-resolution (R7000) spectrum in the red whilst obtaining a longer exposure blue (B3000) spectrum during nights near full moon when the blue sky background was exceptionally high.

We fitted the sodium doublet absorption profile as follows. First, the spectrum was normalised to the local continuum by fitting a quadratic to the SN flux between 10-25 $\AA$ redward or blueward of the doublet centre (5893 $\AA$ ). The absorption profile was fitted as two Gaussians with rest wavelengths set by the doublet wavelengths but with unknown (common) velocity shift and velocity width and (independent) absorption depths. This was done with an mpfit routine in Python, which accounts for variable covariances and returns the appropriate fit values and uncertainties. We show representative examples for fits to data from both gratings in Figure 11. The outcomes for our sodium profile fits are presented in Table A4 in Appendix A, and comprise six successful fits for targets with R7000 spectra, and 13 for R3000 spectra.
The primary quantity we wanted to measure from the sodium absorption feature was its velocity with respect to the local standard of rest at the $\mathrm{SN}$ site. The default local rest velocity was initially set to be the systemic velocity of the $\mathrm{SN}$ host galaxy. In some cases, we detected clear nebular emission lines at the $\mathrm{SN}$ site present in the $\mathrm{SN}$ spectrum-for these cases, the local rest velocity (i.e. the rotational velocity of the host galaxy at the site of the $\mathrm{SN}$ ) was measured from the $\mathrm{H} \alpha$ emission line.

In two cases (SN 2014ao and ASASSN-14jg), no local H $\alpha$ emission was present in the SN spectrum, and the SN sodium velocity differed from the host systemic velocity by more than $100 \mathrm{~km} \mathrm{~s}^{-1}$. To obtain the true local rest velocities for these two SNe Ia, we took advantage of the integral-field data provided by WiFeS, which allows us to measure host galaxy properties (such as velocity) over a broad field of view.

We illustrate this for SN 2014ao in Figure 12: The WiFeS field of view extends from the host galaxy core to the outer edge of its spiral arms in the direction of the SN. We were able to extract the velocity of an $\mathrm{H}$ II region along the $\mathrm{SN}$-host axis and found its velocity differed significantly from that of the host core $\left(\Delta v=174 \pm 10 \mathrm{~km} \mathrm{~s}^{-1}\right)$. As galaxy velocity curves tend to flatten at large radii, we use the $\mathrm{H}$ II region velocity as the local rest velocity of SN 2014ao-a value much closer to the measured sodium absorption velocity.

With the final sodium absorption velocities for our sample, we can inspect the relationship between sodium velocity and other spectroscopic properties of our SN Ia sample. In Figure 13, we plot the silicon absorption ratio $\left(R_{\mathrm{Si}}\right)$, the $\mathrm{HVF}$ absorption strength $\left(R_{\mathrm{HVF}}\right)$, and the absorption strength (i.e. equivalent width) of sodium itself against the velocity of the sodium absorption feature. Based on results of Maguire et al. (2013), we would expect SNe Ia with low $R_{\mathrm{Si}}$ and high $R_{\mathrm{HVF}}$ values (the high stretch, slow declining $\mathrm{SNe}$ Ia) to have a slight excess of blueshifted sodium absorption. Our sample size here is too small (and not well-selected) to make any robust statement about such preferences. However, we note that this analysis was not the explicit objective of our observations, but instead was a supplemental outcome facilitated by the nature of the WiFeS data.

Thus, WiFeS is an excellent instrument for measuring sodium absorption in SNe Ia, a key observable for investigating SN Ia progenitor systems. This is particularly true for observations taken with the R7000 grating, which provides sodium velocity uncertainties of order a few $\mathrm{km} \mathrm{s}^{-1}$, thus enabling a robust classification of the SN as being 'blueshifted' or 'redshifted'. More importantly, perhaps, is the capability of WiFeS to observe a wide field of view around the SN. This enables a measurement of the local systemic velocity at the SN location, even in cases where emission from the host galaxy is weak at the SN location itself.

\subsection{Spectroscopic evolution of SN $2012 \mathrm{dn}$}

The SN with the greatest number of spectroscopic epochs in AWSNAP is SN 2012dn, and we show its AWSNAP 

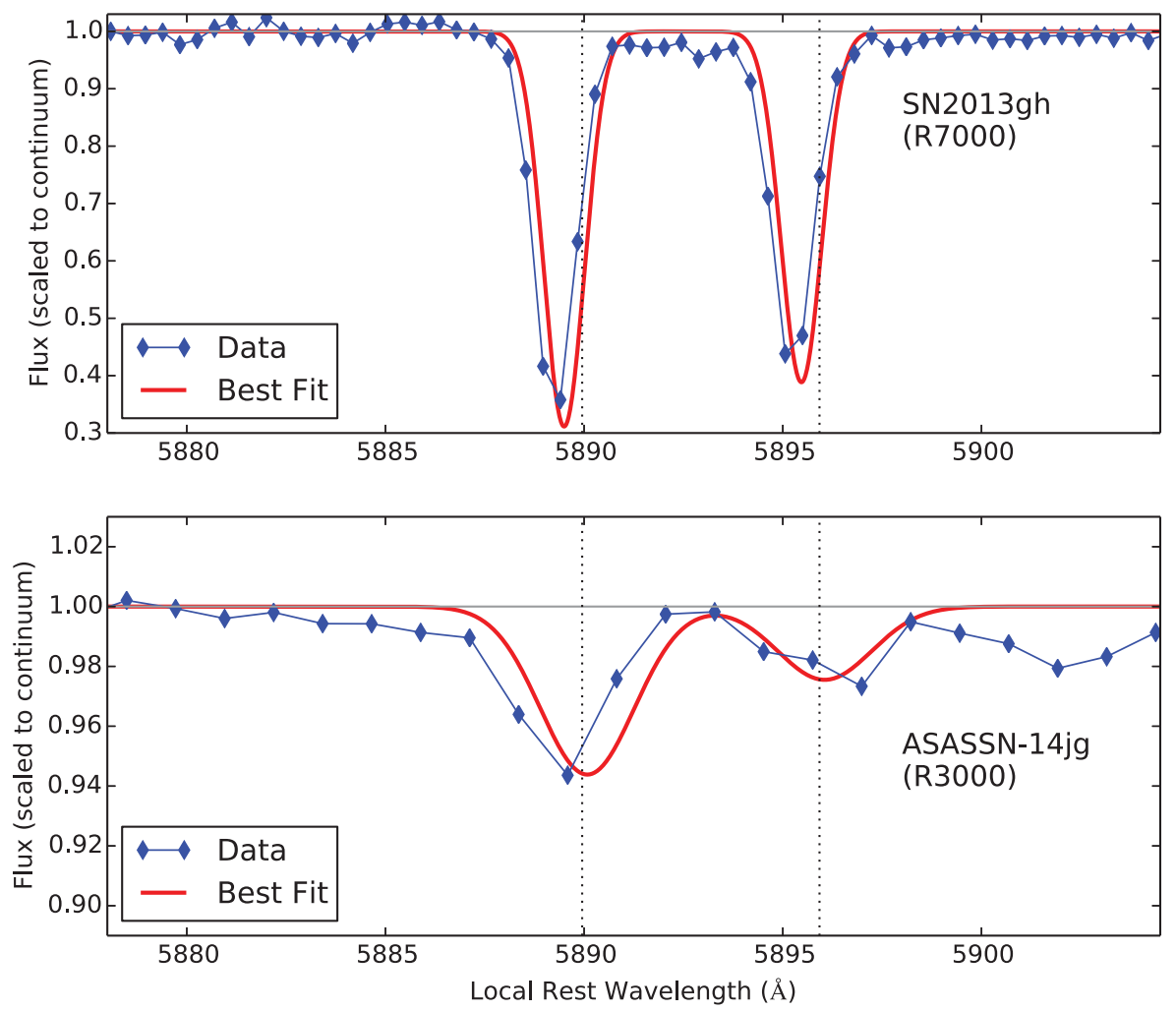

Figure 11. Sodium absorption fit examples for both the R7000 (top) and R3000 (bottom) gratings. Data (which have been normalised to the local continuum fit) are shown as blue diamonds whilst the best fit absorption profile is shown as the solid red curve. For reference, we also mark the continuum level (horizontal black line at value 1.00) and the rest wavelengths of the sodium doublet (vertical dotted gray lines).
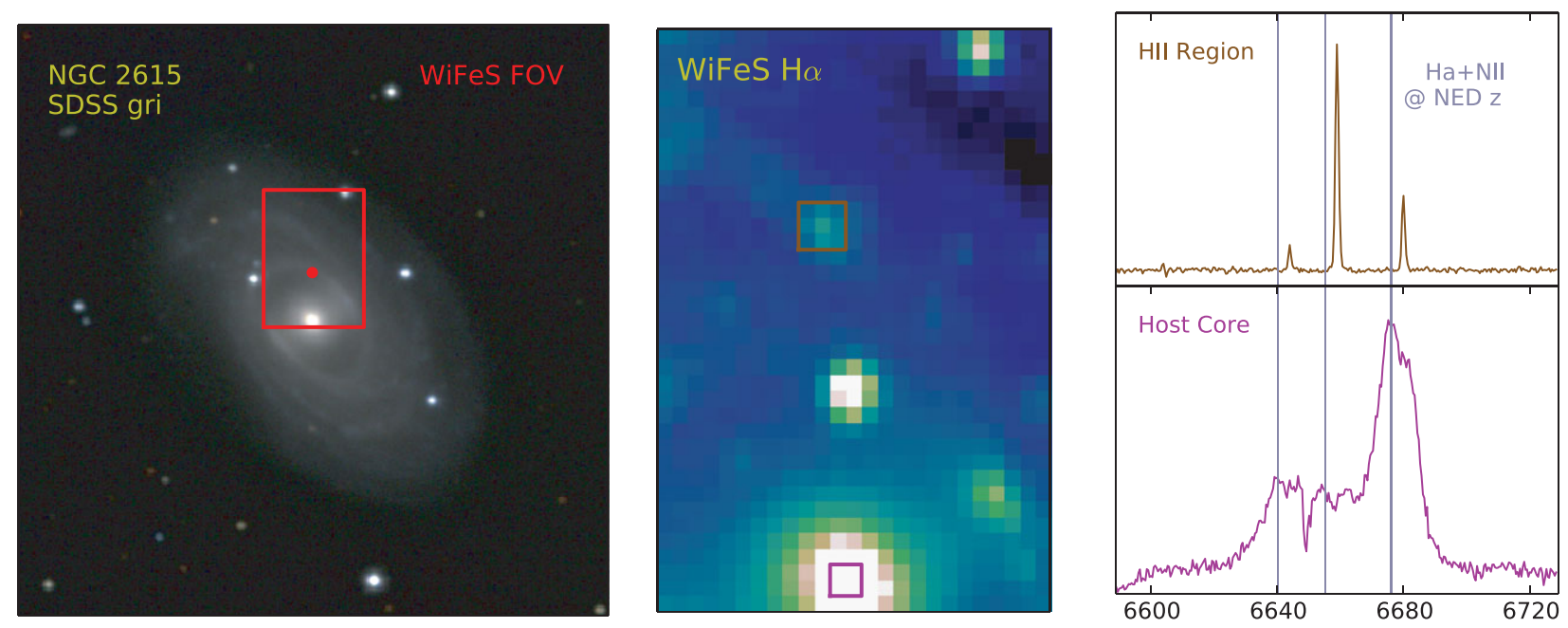

Figure 12. Determination of the local velocity for SN 2014ao in NGC 2615 with WiFeS. Left: SDSS (York et al. 2000) gri colour composite—created with SWARP (Bertin et al. 2002) and STIFF (Bertin 2012)—with the WiFeS field of view (red rectangle) and SN location (red dot) highlighted. Middle: Image of the SN 2014ao WiFeS data cube in the isolated wavelength range within $\pm 6 \AA$ (i.e. $\pm 300 \mathrm{~km} \mathrm{~s}^{-1}$ ) of the wavelength of $\mathrm{H} \alpha$ at the published redshift of NGC 2615, with host core (purple square) and nearby H II region (brown square) highlighted - the SN is the bright object near the centre. Right: Extracted WiFeS spectra of the nearby H II region (top) and host core (bottom) near the H $\alpha+$ NII emission line group, with the expected location of those lines at the published redshift of NGC $2615(z=0.014083$, Theureau et al. 1998a) shown as the vertical gray lines. 

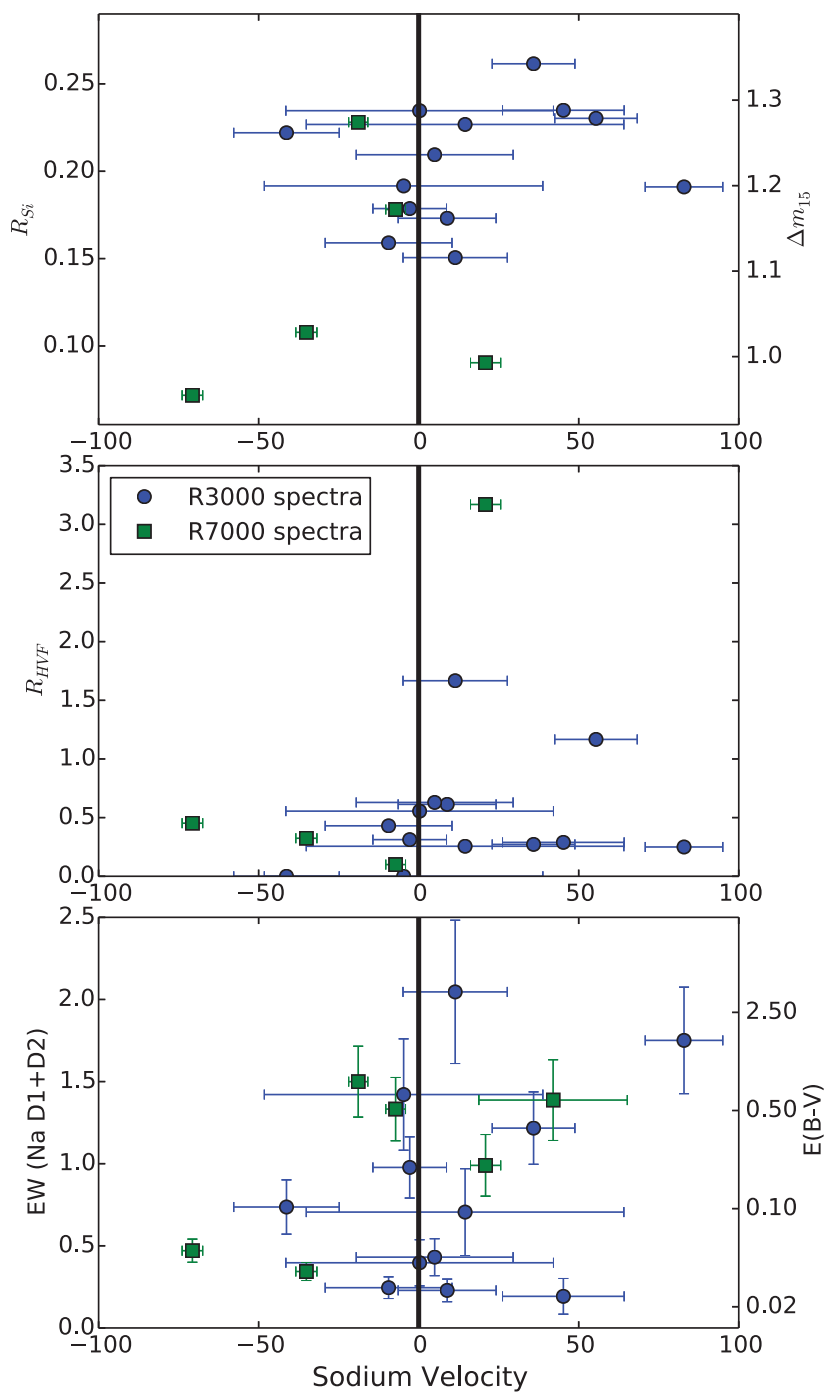

Figure 13. Top: Silicon absorption ratio $R_{\text {Si }}$ plotted against velocity centre of the narrow sodium absorption feature (as in Figure 10 we show the corresponding values of $\Delta m_{15}$, though note the smaller range). Middle: HVF strength $\left(R_{\mathrm{HVF}}\right)$ plotted against sodium absorption velocity. Bottom: Absorption equivalent width of the combined D1+D2 sodium lines plotted against sodium absorption velocity. On the right axis of this panel, we use the relation of Poznanski et al. (2012) to show the reddening values $E(B-V)$ corresponding to the measured sodium equivalent widths if the absorption arises solely from the ISM - though this is unlikely to be true for all SNe Ia (Poznanski et al. 2011; Phillips et al. 2013-see discussion in text). In all panels, higher resolution observations with the R7000 grating are displayed as green squares, whilst lower resolution R3000 observations are shown as blue circles.

spectroscopic time series in Figure 14. SN 2012dn was a spectroscopically peculiar SN Ia whose photometric and spectroscopic evolution was studied extensively by Chakradhari et al. (2014) - they found it to be similar to the "candidate super-Chandrasekhar' SN Ia SN 2006gz (Hicken et al. 2007). For brevity, we will refer to this class of objects as 'superChandra', though we caution the reader that the origin of the extreme luminosity of these objects is still under debate (see, e.g. Taubenberger et al. 2011; Hachinger et al. 2012). We

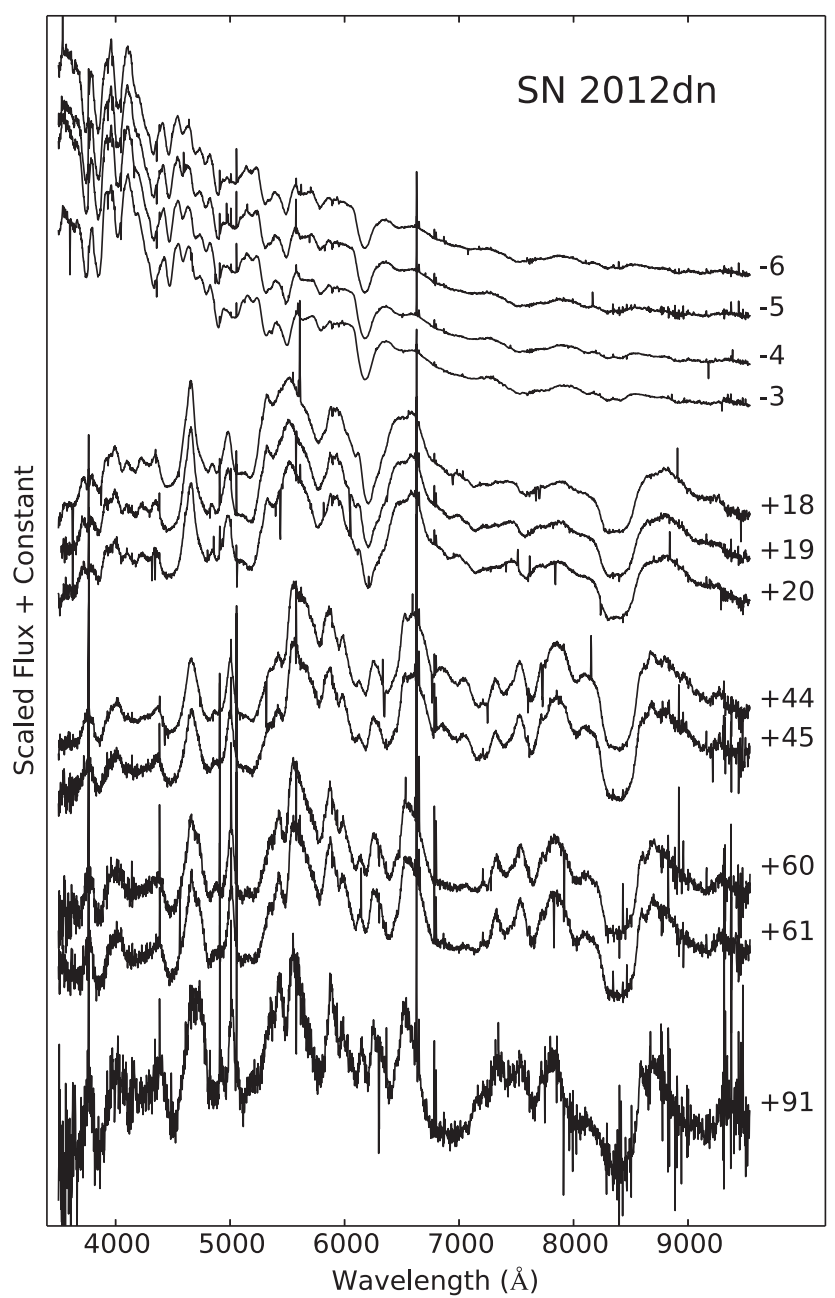

Figure 14. AWSNAP time series of SN 2012dn, labelled by phase with respect to the date of maximum light (2012 July 24, as determined by Chakradhari et al. 2014). Note these observations come from the first semester of AWSNAP when observing time was allocated in multi-night blocks separated sometimes by a month or more.

comment on only a few additional outcomes for SN 2012dn from the AWSNAP data, but refer readers to Chakradhari et al. (2014) and Parrent et al. (2016) for a thorough discussion of this interesting object.

We obtained a very high signal-to-noise spectrum of SN 2012dn at phase $+91 \mathrm{~d}$ (with respect to the date of maximum light 2012 July 24, as determined by Chakradhari et al. 2014). At this epoch, the $\mathrm{SN}$ is beginning to enter the nebular phase when the ejecta become optically thin, revealing emission from the iron group elements (IGEs) near the centre of the SN. Spectra at these epochs provide an excellent diagnostic of the nucleosynthetic products of the SN explosion. In Figure 15, we present our $+91 \mathrm{~d}$ spectrum of SN 2012dn compared to very late spectra of other candidate super-Chandra SNe Ia SN 2007if (Scalzo et al. 2010; Yuan et al. 2010; Taubenberger et al. 2013) and SN 2009dc (Silverman et al. 2011; Taubenberger et al. 2011; Yamanaka et al. 2009; Tanaka et al. 2010; Hachinger et al. 2012; Kamiya et al. 

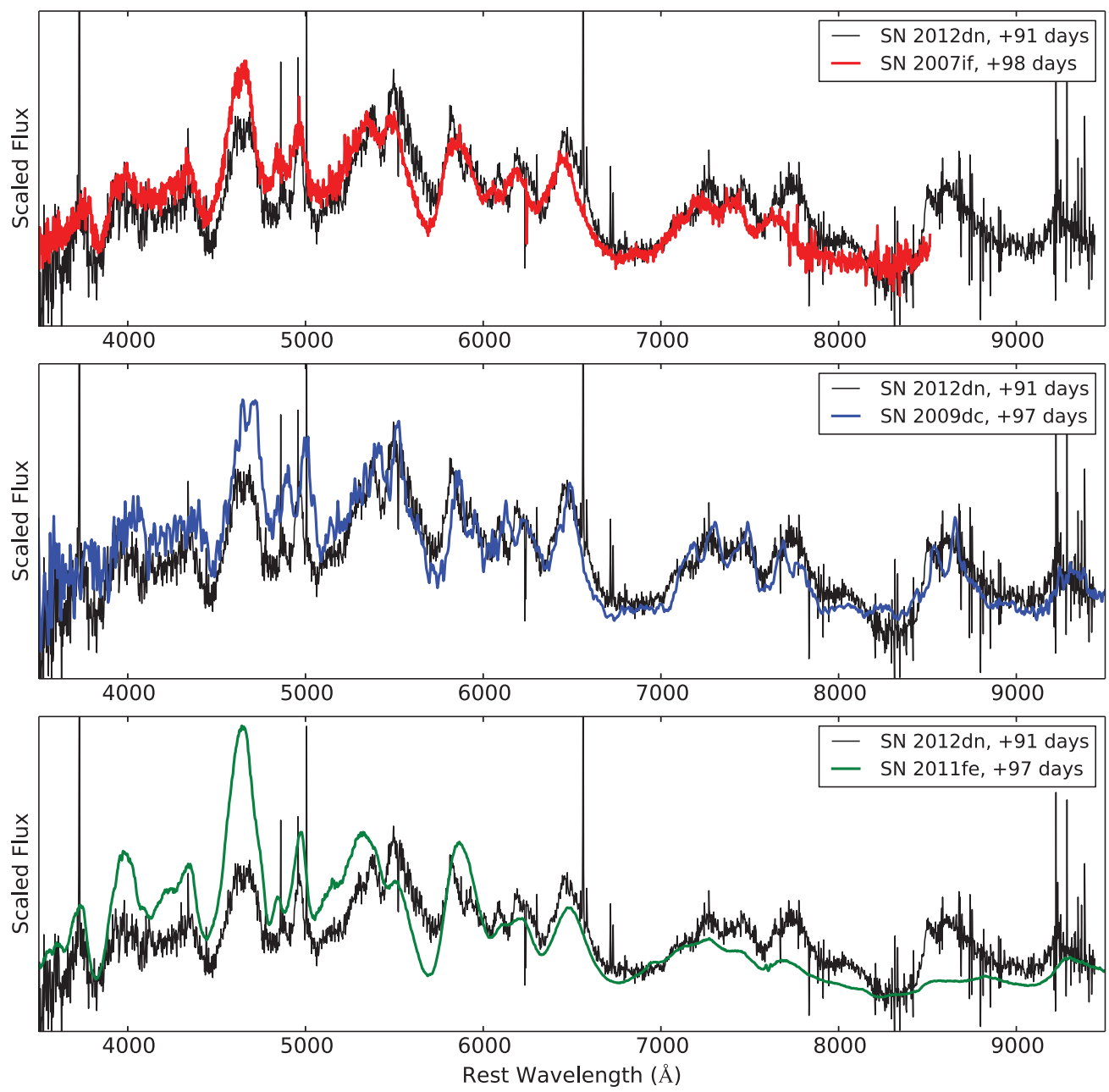

Figure 15. SN 2012dn at its latest AWSNAP epoch (+91 d on 2012 October 23) compared to other candidate superChandra SNe Ia SN 2007if at +98 d (top panel, from Silverman et al. 2011) and SN 2009dc at +97 d (middle panel, from Taubenberger et al. 2011), as well as the normal SN 2011fe (bottom panel, from Pereira et al. 2013).

2012; Taubenberger et al. 2013), as well as the gold standard normal SN Ia SN 2011fe (Nugent et al. 2011; Li et al. 2011b; Parrent et al. 2012; Pereira et al. 2013).

This comparison clearly reveals a strong spectroscopic similarity between SN 2012dn and the candidate superChandrasekhar SNe Ia, and a distinct dissimilarity with SN 2011fe. Perhaps, most prominent is the weaker Fe III line complex at $\sim 4700 \AA$ for the super-Chandra SNe Ia compared to SN 2011fe. This discrepancy is also evident in fully nebular spectra of super-Chandra SNe Ia at $\sim 1$ yr past maximum light, as discussed by Taubenberger et al. (2013). This indicates that the ionisation state of the super-Chandra SNe Ia at these phases is different from normal SNe Ia-whether the diminished Fe III emission arises from a higher or lower average ionisation state remains uncertain.

Additionally, the velocity profile of the emission features in the super-Chandra SNe Ia exhibits a marked difference to that of SN 2011fe (and other normal SNe Ia). The normal SN Ia profile appears very Gaussian (and indeed is generally well fit by a Gaussian profile—Childress et al. 2015), whilst the super-Chandra velocity profile appears sharper at the centre. This is particularly evident for the line features at $\sim 5900$ and $\sim 6300 \AA$, which at these epochs are dominated by Co III. Further spectral modelling of this and other late-phase superChandra spectra may reveal important insights into the structure and composition of the super-Chandra ejecta.

Finally, the high resolution of WiFeS reveals narrow emission lines from the host galaxy of SN 2012dn. We isolated the narrow host galaxy lines using the longest exposure of SN 2012dn, the late-phase observation of 2012 October 23 (+91 d). We fit a simple linear 'continuum' near each emission line by fitting a line to the SN spectrum between 5 and $15 \AA$ away from the line centre on both the blue and red sides of the line. We illustrate this technique and present the SN-subtracted emission features in Figure 16.

In Table 3, we present the measured emission fluxes and errors for the major galaxy emission lines, all of which have been scaled by the observed flux in the $\mathrm{H} \beta$ line. By comparing the observed ratio of the $\mathrm{H} \alpha$ and $\mathrm{H} \beta$ lines to its expected value of 2.87 (the Balmer decrement Osterbrock \& Ferland 


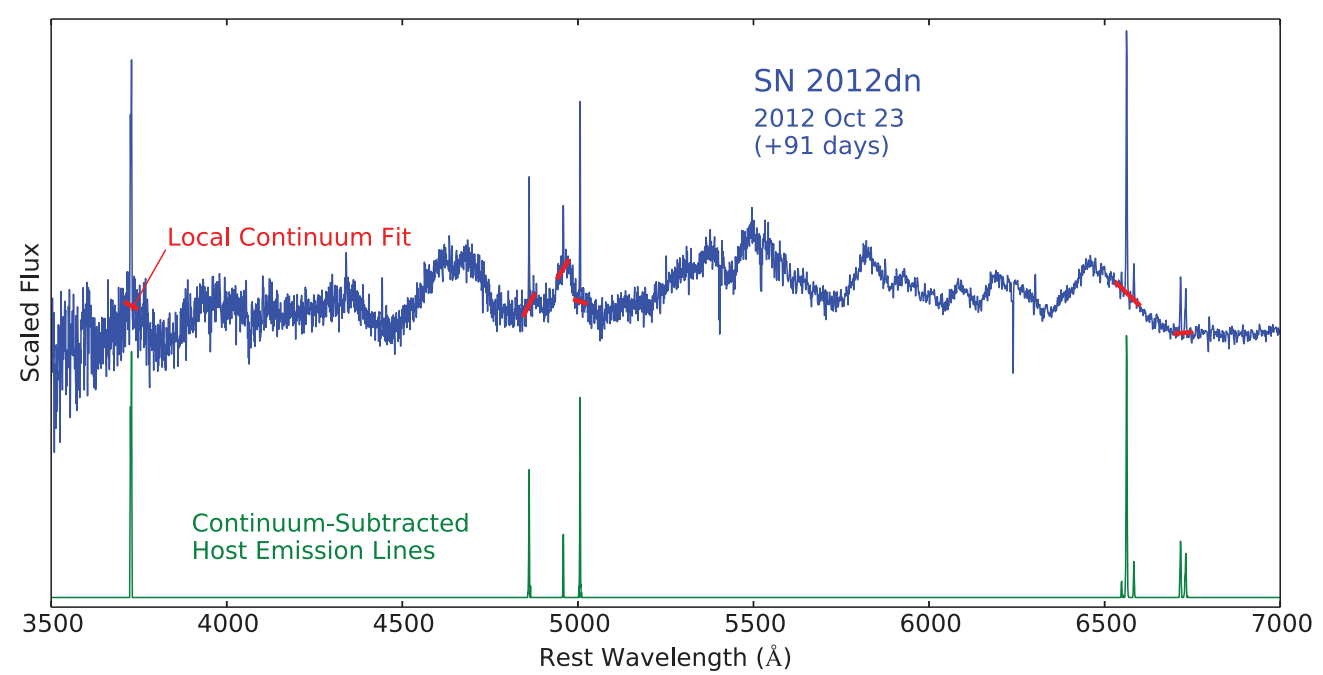

Figure 16. Extraction of host galaxy emission line flux (green) from late SN 2012dn spectrum (blue) using simple linear continuum fits (red).

Table 3. SN 2012dn local emission line fluxes.

\begin{tabular}{lcc}
\hline \hline Line & $\begin{array}{c}F(\lambda) / F(H \beta) \\
(\text { raw })^{a}\end{array}$ & $\begin{array}{c}F(\lambda) / F(H \beta) \\
(\text { de-reddened })^{b}\end{array}$ \\
\hline$[\mathrm{O}$ II $] \lambda \lambda 3727,3730$ & $3.61 \pm 0.24$ & $4.33 \pm 0.29$ \\
$\mathrm{H} \beta$ & $1.00 \pm 0.10$ & $1.00 \pm 0.10$ \\
{$[\mathrm{O}$ III $] \lambda 4959$} & $0.45 \pm 0.09$ & $0.44 \pm 0.09$ \\
{$[\mathrm{O}$ III $] \lambda 5007$} & $1.64 \pm 0.10$ & $1.60 \pm 0.10$ \\
$\mathrm{H} \alpha$ & $3.40 \pm 0.07$ & $2.87 \pm 0.06$ \\
{$[\mathrm{~N} \mathrm{II}] \lambda 6548$} & $0.06 \pm 0.06$ & $0.05 \pm 0.05$ \\
{$[\mathrm{~N} \mathrm{II}] \lambda 6584$} & $0.37 \pm 0.06$ & $0.31 \pm 0.05$ \\
{$[\mathrm{~S} \mathrm{II}] \lambda 6717$} & $0.71 \pm 0.06$ & $0.59 \pm 0.05$ \\
{$[\mathrm{~S} \mathrm{II}] \lambda 6731$} & $0.58 \pm 0.06$ & $0.48 \pm 0.05$ \\
\hline \hline
\end{tabular}

${ }^{a}$ Observer frame fluxes, scaled to $\mathrm{H} \beta$.

${ }^{b}$ De-reddened using Balmer decrement reddening of $E(B-V)=0.17$ so that $F(H \beta)=F(H \alpha) / 2.87$ with a CCM reddening law, and scaled to the de-reddened flux of $\mathrm{H} \beta$.

2006), we can determine the amount of reddening in the $\mathrm{H}$ II regions giving rise to the host emission lines. We use the Cardelli, Clayton, \& Mathis (1989) to find a host reddening of $E(B-V)=0.17 \pm 0.10$, a value remarkably similar to the $\mathrm{SN}$ reddening of $E(B-V)=0.18$ (Milky Way plus host) determined by Chakradhari et al. (2014). We correct the host emission line fluxes for the value $E(B-V)=0.17$ and report the corrected values (which we also re-scale to the de-reddened $\mathrm{H} \beta$ flux) in Table 3 .

With the de-reddened host galaxy emission line fluxes, we calculate a gas-phase metallicity at the site of SN 2012dn. The N2 method of Pettini \& Pagel (2004, hereafter PP04) yields $12+\log (O / H)=8.29 \pm 0.04$, whilst the O3N2 method of PP04 yields $12+\log (O / H)=8.35 \pm 0.03$. The former value is remarkably close to the estimated site metallicity for SN $2006 \mathrm{gz}$ of $12+\log (O / H)=8.26$ calculated by Khan et al. (2011) using the same metallicity method. If we convert the O3N2 value to the Tremonti et al. (2004) scale us- ing the formulae of Kewley \& Ellison (2008) as was done in Childress et al. (2011), we measure $12+\log (O / H)_{T 04}=$ $8.51 \pm 0.04$.

Comparing the above values to the solar oxygen abundance of $12+\log (O / H)_{\odot}=8.69$ (Asplund et al. 2009), we find the site metallicity for SN $2012 \mathrm{dn}$ is in the range 40-65\% solar, depending on the chosen metallicity calibration. This sub-solar metallicity value is consistent with the previously reported trend for super-Chandra $\mathrm{SNe}$ Ia to prefer low metallicity environments (Taubenberger et al. 2011; Childress et al. 2011; Khan et al. 2011).

\section{CONCLUSIONS}

This work marks the primary data release for the AWSNAP, comprising 357 distinct spectra of 175 unique SNe. These data were collected using the WiFeS on the ANU 2.3-m telescope during 82 nights of observing over a 3-yr period from mid-2012 to mid-2015.

The AWSNAP spectroscopy sample is comparable in size to other SN spectra data releases, and its composition of SN types is roughly in line with expectations for a magnitudelimited SN search. The phase coverage of the AWSNAP SN Ia sample is comparable to other published SN Ia spectroscopy datasets (for SNe Ia with multiple epochs of observation), with the inclusion of more SNe Ia with a single observation (i.e. classification spectra only).

We presented some analyses of the AWSNAP SN Ia sample, including some results uniquely enabled by the fine wavelength resolution available with WiFeS. We measured broad absorption features in SN Ia spectra at maximum light, including the ratio of silicon absorption features $R_{\mathrm{Si}}$ and the strength of HVFs $R_{\mathrm{HVF}}$. Additionally, we measured the strength and velocity of narrow sodium absorption features, including some cases where the integral-field nature of the 
instrument allowed us to measure the local systemic velocity within the $\mathrm{SN}$ host galaxy. Some expected feature trends, such as a correlation between $R_{\mathrm{HVF}}$ and $R_{\mathrm{Si}}$, were recovered in our data set. The nature of sodium absorption in our sample was limited by small number statistics. Finally, we presented our observations of the candidate super-Chandrasekhar SN Ia SN 2012dn, and used narrow host galaxy emission features to show the $\mathrm{SN}$ site exhibits sub-solar metallicity.

The WiFeS instrument presents several unique advantages for the study of transients, particularly owing to its comparatively narrow velocity resolution $\left(\sigma_{\mathrm{v}} \sim 45 \mathrm{~km} \mathrm{~s}^{-1}\right)$. It has previously been employed in the study of $\mathrm{SNe}$ with strong narrow emission features such as SN 2009ip (Fraser et al. 2013, 2015), SN 2012ca (Inserra et al. 2014, 2016), and SN 2013fc (Kangas et al. 2016). Here, we also demonstrated that the fine velocity resolution allows for the measurement of narrow $a b$ sorption features, particularly sodium absorption in SNe Ia. The higher resolution of WiFeS also frequently revealed narrow host galaxy emission features at the site of the SN, which can at times be used to determine a SN site metallicity (as we showed for SN 2012dn). Finally, the integral field nature of WiFeS allowed us to measure the local host galaxy rotational velocity at the site of several $\mathrm{SNe}$, even when there was no emission directly at the SN location. Thus, WiFeS is an instrument capable of not only standard SN spectroscopic observations, but also a unique suite of capabilities not commonly found in transient follow-up instruments.

\section{ACKNOWLEDGEMENTS}

We are very grateful for the excellent technical support staff for the ANU 2.3-m telescope and the WiFeS instrument: Peter Verwayen, Ian Adams, Peter Small, Ian Price, Peter Young, and Jon Nielsen. We thank the ANU telescope time allocation committee who continue to support the observations presented herein. We also thank Julie Banfield, Michael Ireland, Stefan Keller, Lisa Kewley, Jeremy Mould, Chris Owen, Aaron Rizzuto, Dary Ruiz, and Tian-Tian Yuan for additional observations. We also thank the anonymous referee for very helpful comments.

This research was conducted by the Australian Research Council Centre of Excellence for All-sky Astrophysics (CAASTRO), through project number CE110001020. IRS was supported by Australian Research Council Laureate Grant FL0992131. This research has made use of the NASA/IPAC Extragalactic Database (NED) which is operated by the Jet Propulsion Laboratory, California Institute of Technology, under contract with the National Aeronautics and Space Administration. This research has made use of NASA's Astrophysics Data System (ADS).

\section{REFERENCES}

Aldering, G., et al. 2002, in SPIE Conf. Ser., Vol. 4836, Survey and Other Telescope Technologies and Discoveries, eds. J. A. Tyson \& S. Wolff (Waikoloa: SPIE), 61, doi:10.1117/12.458107

Asplund, M., Grevesse, N., Sauval, A. J., \& Scott, P. 2009, ARA\&A, 47, 481

Bailey, S., et al.2009, A\&A, 500, L17

Baltay, C., et al.2013, PASP, 125, 683
Barbon, R., Benetti, S., Rosino, L., Cappellaro, E., \& Turatto, M. 1990, A\&A, 237, 79

Benetti, S., et al.2005, ApJ, 623, 1011

Bertin, E. 2012, in ASP Conf. Ser., Vol. 461, Astronomical Data Analysis Software and Systems XXI, eds. P. Ballester, D. Egret, \& N. P. F. Lorente (San Francisco: ASP), 263

Bertin, E., Mellier, Y., Radovich, M., Missonnier, G., Didelon, P., \& Morin, B. 2002, in ASP Conf. Ser., Vol. 281, Astronomical Data Analysis Software and Systems XI, eds. D. A. Bohlender, D. Durand, \& T. H. Handley (San Francisco: ASP), 228

Bessell, M. S. 1999, PASP, 111, 1426

Bianco, F. B., et al. 2014, ApJS, 213, 19

Blagorodnova, N., Koposov, S. E., Wyrzykowski, Ł., Irwin, M., \& Walton, N. A. 2014, MNRAS, 442, 327

Blagorodnova, N., Van Velzen, S., Harrison, D. L., Koposov, S., Mattila, S., Campbell, H., Walton, N. A., \& Wyrzykowski, Ł. 2016, MNRAS, 455, 603

Blondin, S., Mandel, K. S., \& Kirshner, R. P. 2011, A\&A, 526, A81

Blondin, S., \& Tonry, J. L. 2007, ApJ, 666, 1024

Blondin, S., et al. 2012, AJ, 143, 126

Bongard, S., Baron, E., Smadja, G., Branch, D., \& Hauschildt, P. H. 2006, ApJ, 647, 513

Bongard, S., Baron, E., Smadja, G., Branch, D., \& Hauschildt, P. H. 2008, ApJ, 687, 456

Branch, D., Dang, L. C., \& Baron, E. 2009, PASP, 121, 238

Branch, D., et al. 2006, PASP, 118, 560

Bronder, T. J., et al. 2008, A\&A, 477, 717

Brown, T. M., et al. 2013, PASP, 125, 1031

Cardelli, J. A., Clayton, G. C., \& Mathis, J. S. 1989, ApJ, 345, 245

Chakradhari, N. K., Sahu, D. K., Srivastav, S., \& Anupama, G. C. 2014, MNRAS, 443, 1663

Childress, M., et al. 2011, ApJ, 733, 3

Childress, M. J., Filippenko, A. V., Ganeshalingam, M., \& Schmidt, B. P. 2014b, MNRAS, 437, 338

Childress, M. J., Vogt, F. P. A., Nielsen, J., \& Sharp, R. G. 2014a, Ap\&SS, 349, 617

Childress, M. J., et al. 2015, MNRAS, 454, 3816

Cortese, L., et al. 2008, MNRAS, 383, 1519

da Costa, L. N., Pellegrini, P. S., Davis, M., Meiksin, A., Sargent, W. L. W., \& Tonry, J. L. 1991, ApJS, 75, 935

Donzelli, C. J., \& Pastoriza, M. G. 2000, AJ, 120, 189

Dopita, M., Hart, J., McGregor, P., Oates, P., Bloxham, G., \& Jones, D. 2007, Ap\&SS, 310, 255

Dopita, M., et al. 2010, Ap\&SS, 327, 245

Drake, A. J., et al. 2009, ApJ, 696, 870

Filippenko, A. V. 1997, ARA\&A, 35, 309

Filippenko, A. V., et al. 1992a, AJ, 104, 1543

Filippenko, A. V., et al. 1992b, ApJL, 384, L15

Folatelli, G., et al. 2013, ApJ, 773, 53

Foley, R. J., et al. 2013, ApJ, 767, 57

Fraser, M., et al. 2013, MNRAS, 433, 1312

Fraser, M., et al. 2015, MNRAS, 453, 3886

Ganeshalingam, M., et al. 2010, ApJS, 190, 418

Graur, O., Bianco, F. B., Modjaz, M., Maoz, D., Shivvers, I., Filippenko, A. V., \& Li, W. 2015, preprint (arXiv:1509.08432)

Grogin, N. A., Geller, M. J., \& Huchra, J. P. 1998, ApJS, 119, 277

Hachinger, S., Mazzali, P. A., \& Benetti, S. 2006, MNRAS, 370, 299

Hachinger, S., Mazzali, P. A., Tanaka, M., Hillebrandt, W., \& Benetti, S. 2008, MNRAS, 389, 1087 
Hachinger, S., Mazzali, P. A., Taubenberger, S., Fink, M., Pakmor, R., Hillebrandt, W., \& Seitenzahl, I. R. 2012, MNRAS, 427, 2057

Hatano, K., Branch, D., Lentz, E. J., Baron, E., Filippenko, A. V., \& Garnavich, P. M. 2000, ApJL, 543, L49

Hicken, M., Garnavich, P. M., Prieto, J. L., Blondin, S., DePoy, D. L., Kirshner, R. P., \& Parrent, J. 2007, ApJL, 669, L17

Holoien, T. W.-S., et al. 2016, MNRAS

Inserra, C., et al. 2013, ApJ, 770, 128

Inserra, C., et al. 2014, MNRAS, 437, L51

Inserra, C., et al. 2016, MNRAS, 459, 2721

Kaiser, N., et al. 2010, in Ground-based and Airborne Telescopes III, SPIE Proc. 7733, eds. L.M. Stepp, R. Gilmozzi, \& H.J. Hall (San Diego: SPIE), 77330E

Kamiya, Y., Tanaka, M., Nomoto, K., Blinnikov, S. I., Sorokina, E. I., \& Suzuki, T. 2012, ApJ, 756, 191

Kangas, T., et al. 2016, MNRAS, 456, 323

Keller, S. C., et al. 2007, PASA, 24, 1

Kelson, D. D. 2003, PASP, 115, 688

Kewley, L. J., \& Ellison, S. L. 2008, ApJ, 681, 1183

Khan, R., Stanek, K. Z., Stoll, R., \& Prieto, J. L. 2011, ApJL, 737, 24

Klotz, A., Boer, M., Atteia, J.-L., Gendre, B., Le Borgne, J.-F., Frappa, E., Vachier, F., \& Berthier, J. 2013, Msngr, 151, 6

Law, N. M., et al. 2009, PASP, 121, 1395

Li, W., et al. 2011a, MNRAS, 412, 1441

Li, W., et al. 2011b, Nature, 480, 348

Lipunov, V. M., et al. 2004, AN, 325, 580

Liu, Y.-Q., Modjaz, M., Bianco, F. B., \& Graur, O. 2016, ApJ, 827, 90

Maguire, K., et al. 2012, MNRAS, 426, 2359

Maguire, K., et al. 2013, MNRAS, 436, 222

Maguire, K., et al. 2014, MNRAS, 444, 3258

Matheson, T., et al. 2008, AJ, 135, 1598

Modjaz, M., et al. 2014, AJ, 147, 99

Nugent, P., Phillips, M., Baron, E., Branch, D., \& Hauschildt, P. 1995, ApJL, 455, L147

Nugent, P. E., et al. 2011, Nature, 480, 344

Ogando, R. L. C., Maia, M. A. G., Pellegrini, P. S., \& da Costa, L. N. 2008, AJ, 135, 2424

Oke, J. B. 1990, AJ, 99, 1621

Osterbrock, D. E., \& Ferland, G. J. 2006, Astrophysics of gaseous nebulae and active galactic nuclei (Sausalito: University Science Books)

Pan, Y.-C., Sullivan, M., Maguire, K., Gal-Yam, A., Hook, I. M., Howell, D. A., Nugent, P. E., \& Mazzali, P. A. 2015, MNRAS, 446,354

Parrent, J. T., et al. 2012, ApJL, 752, L26

Parrent, J. T., et al. 2016, MNRAS, 457, 3702

Patat, F., et al. 2007, Science, 317, 924

Pereira, R., et al. 2013, A\&A, 554, A27

Pettini, M., \& Pagel, B. E. J. 2004, MNRAS, 348, L59

Phillips, M. M., et al. 2013, ApJ, 779, 38

Pignata, G., et al. 2009, in AIP Conf. Ser., Vol. 1111, eds. G. Giobbi, A. Tornambe, G. Raimondo, M. Limongi, L. A. Antonelli, N. Menci, \& E. Brocato (Sicily: AIP), 551

Planck Collaboration et al. 2011, A\&A, 536, A1

Poznanski, D., Ganeshalingam, M., Silverman, J. M., \& Filippenko, A. V. 2011, MNRAS, 415, L81

Poznanski, D., Prochaska, J. X., \& Bloom, J. S. 2012, MNRAS, 426,1465
Quimby, R. M. 2006, PhD thesis, The University of Texas at Austin Rau, A., et al. 2009, PASP, 121, 1334

Salim, S., et al. 2007, ApJS, 173, 267

Sandage, A., \& Tammann, G. A. 1981, A revised Shapley-Ames Catalog of bright galaxies, Carnegie Institution of Washington Pub. 635 (Washington: Carnegie Institution)

Scalzo, R., et al. 2012, ApJ, 757, 12

Scalzo, R. A., et al. 2010, ApJ, 713, 1073

Shappee, B. J., et al. 2014, ApJ, 788, 48

Silverman, J. M., Ganeshalingam, M., Li, W., Filippenko, A. V., Miller, A. A., \& Poznanski, D. 2011, MNRAS, 410, 585

Silverman, J. M., Ganeshalingam, M., Li, W., \& Filippenko, A. V. 2012a, MNRAS, 425, 1889

Silverman, J. M., Kong, J. J., \& Filippenko, A. V. 2012b, MNRAS, 425,1819

Silverman, J. M., Vinkó, J., Marion, G. H., Wheeler, J. C., Barna, B., Szalai, T., Mulligan, B. W., \& Filippenko, A. V. 2015, MNRAS, 451,1973

Silverman, J. M., et al. 2012c, MNRAS, 425, 1789

Simon, J. D., et al. 2009, ApJ, 702, 1157

Smartt, S. J., et al. 2015, A\&A, 579, A40

Sternberg, A., et al. 2011, Science, 333, 856

Sternberg, A., et al. 2014, MNRAS, 443, 1849

Stritzinger, M., Suntzeff, N. B., Hamuy, M., Challis, P., Demarco, R., Germany, L., \& Soderberg, A. M. 2005, PASP, 117, 810

Tanaka, M., et al. 2010, ApJ, 714, 1209

Taubenberger, S., et al. 2011, MNRAS, 412, 2735

Taubenberger, S., et al. 2013, MNRAS, 432, 3117

Theureau, G., Bottinelli, L., Coudreau-Durand, N., Gouguenheim, L., Hallet, N., Loulergue, M., Paturel, G., \& Teerikorpi, P. 1998a, A\&AS, 130, 333

Theureau, G., Bottinelli, L., Coudreau-Durand, N., Gouguenheim, L., Hallet, N., Loulergue, M., Paturel, G., \& Teerikorpi, P. 1998b, A\&AS, 130, 333

Tomasella, L., et al. 2014, AN, 335, 841

Tremonti, C. A., et al. 2004, ApJ, 613, 898

Turatto, M., Benetti, S., \& Cappellaro, E. 2003, in From Twilight to Highlight: The Physics of Supernovae, Proc. of the ESO/MPA/MPE Workshop, eds. W. Hillebrandt \& B. Leibundgut (Garching: ESO), 200

van Dokkum, P. G. 2001, PASP, 113, 1420

Wang, X., et al. 2009, ApJL, 699, L139

Wyrzykowski, L., Hodgkin, S., Blogorodnova, N., Koposov, S., \& Burgon, R. 2012, preprint (arXiv:1210.5007)

Wyrzykowski, Ł., et al. 2014, ACTA, 64, 197

Yamanaka, M., et al. 2009, ApJL, 707, L118

Yaron, O., \& Gal-Yam, A. 2012, PASP, 124, 668

York, D. G., et al. 2000, AJ, 120, 1579

Yuan, F. 2010, PhD thesis, University of Michigan

Yuan, F., et al. 2010, ApJ, 715, 1338

Zhao, X., et al. 2015, ApJS, 220, 20

\section{APPENDIX A1: TARGET AND OBSERVATION DETAILS}

In this Appendix, we present the observational metadata for the AWSNAP data release, as well as sodium fit results and host (or local) redshifts used in the sodium fitting.

AWSNAP targets: Table A1 presents the discovery and classification details for the full list of 175 objects featured in the AWSNAP 
Table A1. All AWSNAP Targets.

\begin{tabular}{|c|c|c|c|c|c|c|c|c|c|c|c|}
\hline \multirow[b]{2}{*}{ SN } & \multicolumn{5}{|c|}{ Discovery } & \multicolumn{6}{|c|}{ Classification } \\
\hline & RA & DEC & Ref. $^{a}$ & Date & Group $^{b}$ & Ref. & Date & Group $^{c}$ & SN type & Redshift & Phase $^{d}$ \\
\hline SN 2009ip & $22: 23: 08.1$ & $-28: 56: 35$ & A4334 & 20120724 & CRTS & A4338 & 20120826 & Foley & SNIIn & 0.005944 & (unk) \\
\hline SN 2012ca & $18: 41: 06.5$ & $-41: 47: 38$ & C3101 & 20120426 & Parker & A4076 & 20120429 & PESSTO & SNIIn & 0.019000 & (unk) \\
\hline SN 2012dj & $23: 14: 47.2$ & $-43: 36: 22$ & C3167 & 20120702 & Parker & C3167 & 20120706 & Parrent & $\mathrm{SNIb} / \mathrm{c}$ & 0.005324 & +0 \\
\hline SN 2012dn & $20: 23: 36.3$ & $-28: 16: 43$ & C3174 & 20120708 & Parker & A4253 & 20120712 & SNF & SNIa & 0.010187 & -10 \\
\hline SN 2012dt & $00: 56: 38.1$ & $-09: 53: 59$ & C3188 & 20120718 & Parker & A4269 & 20120721 & PTF & SNII & 0.018900 & +9 \\
\hline SN 2012dy & $21: 18: 49.5$ & $-57: 38: 42$ & C3197 & 20120804 & Bock & C3197 & 20120805 & Milisavljevic & SNII & 0.010300 & (young) \\
\hline SN 2012ec & $02: 45: 59.9$ & $-07: 34: 15$ & C3201 & 20120811 & Monard & A4306 & 20120812 & AWSNAP & SNII & 0.004693 & (young) \\
\hline SN 2012eq & 01:00:14.5 & $-30: 48: 26$ & C3223 & 20120827 & CRTS & A4362 & 20120907 & PESSTO & SNIa & 0.032230 & +45 \\
\hline SN 2012eu & 03:13:04.2 & $-08: 23: 24$ & C3231 & 20120827 & CRTS & C3231 & 20120904 & Yang & SNIa & 0.030000 & +30 \\
\hline SN 2012fr & 03:33:37.1 & $-36: 07: 28$ & A4523 & 20121027 & TAROT & A4525 & 20121028 & AWSNAP & SNIa & 0.005400 & -11 \\
\hline SN 2012hr & $06: 21: 37.7$ & $-59: 42: 39$ & C3346 & 20121216 & Marples & A4663 & 20121220 & Morrell & SNIa & 0.008000 & -8 \\
\hline SN 2013K & $17: 39: 33.1$ & $-85: 18: 18$ & C3391 & 20130120 & Parker & A4754 & 20130121 & PESSTO & SNII & 0.008000 & +2 \\
\hline SN 2013aa & $14: 32: 33.3$ & $-44: 13: 20$ & C3416 & 20130213 & Parker & A4817 & 20130213 & LCOGT & SNIa & 0.003999 & -3 \\
\hline SN 2013ai & $06: 16: 18.4$ & $-21: 22: 23$ & C3431 & 20130226 & Conseil & A4851 & 20130302 & PESSTO & SNII & 0.009000 & +0 \\
\hline SN 2013aj & $13: 54: 00.8$ & $-07: 55: 37$ & C3434 & 20130303 & Contini & A4852 & 20130303 & PESSTO & SNIa & 0.009000 & -7 \\
\hline SN 2013am & $11: 18: 56.3$ & $+13: 03: 56$ & C3440 & 20130321 & Sugano & A4909 & 20130322 & ASP & SNII & 0.002692 & (young) \\
\hline SN 2013ao & $11: 44: 44.7$ & $-20: 31: 41$ & C3442 & 20130304 & CRTS & A4863 & 20130305 & PESSTO & SNIa & 0.030000 & -7 \\
\hline SN 2013bj & 14:04:19.9 & $-07: 03: 02$ & C3482 & 20130404 & ISSP & A4975 & 20130412 & AWSNAP & SNII & 0.030000 & +44 \\
\hline SN 2013bk & 09:40:47.6 & $-05: 26: 07$ & C3483 & 20130407 & CRTS & A4975 & 20130412 & AWSNAP & SNIc & 0.020000 & +3 \\
\hline SN 2013by & $16: 59: 01.5$ & $-60: 11: 22$ & $\mathrm{C} 3506$ & 20130423 & Parker & C3506 & 20130424 & Morrell & SNII & 0.003816 & (young) \\
\hline SN 2013bz & $13: 26: 50.7$ & $-10: 01: 39$ & $\mathrm{C} 3507$ & 20130421 & CRTS & C3507 & 20130430 & Chen & SNIa-SC & 0.015000 & +0 \\
\hline SN 2013cg & $09: 26: 57.3$ & $-24: 47: 01$ & C3517 & 20130506 & CHASE & A5057 & 20130508 & LCOGT & SNIa & 0.007952 & -7 \\
\hline SN 2013cs & $13: 15: 14.2$ & $-17: 57: 56$ & C3533 & 20130512 & LSQ & A5067 & 20130514 & LSQ & SNIa & 0.009240 & -7 \\
\hline SN 2013cy & $22: 10: 33.5$ & $-22: 39: 48$ & C3546 & 20130531 & Monard/LSQ & A5100 & 20130603 & AWSNAP & SNIa & 0.034000 & +0 \\
\hline SN 2013cz & $19: 49: 32.5$ & $-70: 12: 59$ & C 3547 & 20130507 & Parker & A5101 & 20130603 & AWSNAP & SNII & 0.015000 & +21 \\
\hline SN 2013df & $12: 26: 28.1$ & $+31: 13: 50$ & C3557 & 20130607 & ISSP & C3557 & 20130610 & Cenko & SNIIb & 0.002388 & (young) \\
\hline SN 2013dh & $15: 30: 00.3$ & $+12: 59: 27$ & C3561 & 20130612 & LOSS & C3561 & 20130614 & Cenko & SNIa-91T & 0.013356 & -5 \\
\hline SN 2013du & 01:24:13.6 & $-34: 43: 27$ & C3579 & 20130623 & Parker & A5194 & 20130704 & AWSNAP & SNII & 0.019000 & +30 \\
\hline SN 2013ef & $01: 55: 20.2$ & $+06: 36: 35$ & A5193 & 20130704 & ASAS-SN & A5194 & 20130704 & AWSNAP & SNIa & 0.015000 & +0 \\
\hline SN 2013ej & $01: 36: 48.2$ & $+15: 45: 35$ & C3606 & 20130725 & LOSS & A5228 & 20130727 & LCOGT & SNII & 0.002192 & (young) \\
\hline SN 2013ek & $20: 57: 54.2$ & $-51: 52: 10$ & C3607 & 20130724 & Parker & A5227 & 20130725 & LCOGT & SNIb & 0.016000 & +5 \\
\hline SN 2013er & 13:07:51.2 & $-00: 51: 54$ & C3619 & 20130729 & Parker & A5238 & 20130731 & AWSNAP & SNIa & 0.018052 & -4 \\
\hline SN 2013fd & $01: 33: 59.6$ & $-34: 23: 38$ & C3645 & 20130827 & Parker & A5345 & 20130829 & PESSTO & SNII & 0.013000 & (young) \\
\hline SN 2013fj & $22: 15: 28.3$ & $+15: 34: 06$ & C3654 & 20130907 & ISSP & C3654 & 20130913 & ASP & SNIa & 0.033570 & +4 \\
\hline SN 2013fq & $19: 59: 06.7$ & $-55: 55: 36$ & C3665 & 20130915 & Parker & A5400 & 20130918 & Hsiao & SNIIb & 0.011300 & +0 \\
\hline SN 2013fs & $23: 19: 44.5$ & $+10: 10: 38$ & C3671 & 20131006 & Itagaki & A5527 & 20131008 & AWSNAP & SNII & 0.012000 & (young) \\
\hline SN $2013 \mathrm{ft}$ & $23: 41: 36.9$ & $+03: 43: 16$ & C3672 & 20130913 & LOSS & A5455 & 20131008 & AWSNAP & SNII & 0.009600 & +13 \\
\hline SN 2013fw & 21:13:44.1 & $+13: 34: 50$ & C3681 & 20131022 & Jin,Gao & A5507 & 20131024 & LCOGT & SNIa & 0.016952 & -14 \\
\hline SN 2013gh & $22: 02: 21.8$ & $-18: 55: 00$ & C3706 & 20130808 & LOSS & A5262 & 20130811 & LCOGT & SNIa & 0.008800 & -7 \\
\hline SN 2013gi & $06: 23: 48.2$ & $-65: 19: 43$ & C3714 & 20131113 & Parker & A5582 & 20131114 & AWSNAP & SNIa & 0.030100 & +10 \\
\hline SN 2013gq & $19: 59: 07.8$ & $-55: 55: 03$ & C3730 & 20130325 & LOSS & A4940 & 20130402 & PESSTO & SNIa & 0.014000 & +0 \\
\hline SN 2013gr & $01: 46: 28.5$ & $-58: 40: 25$ & C3733 & 20131128 & Marples & A5612 & 20131130 & Hsiao & SNIax & 0.007000 & +0 \\
\hline SN 2013hh & $11: 29: 04.8$ & $+17: 14: 08$ & C3754 & 20131111 & TAROT & A5656 & 20131212 & AWSNAP & SNIa-91T & 0.012000 & -2 \\
\hline SN 2013hj & 09:12:05.7 & $-15: 25: 39$ & C3757 & 20131212 & CHASE & C3757 & 20131213 & ASP & SNII & 0.006900 & (young) \\
\hline
\end{tabular}


Table A1. Continued.

Classification

\begin{tabular}{|c|c|c|c|c|c|c|c|c|c|c|c|}
\hline $\mathrm{SN}$ & RA & DEC & Ref. $^{a}$ & Date & Group $^{b}$ & Ref. & Date & Group $^{c}$ & SN type & Redshift & Phase $^{d}$ \\
\hline SN 2013hn & $13: 50: 52.3$ & $-30: 16: 54$ & C3762 & 20131213 & Kot+ & C3762 & 20131217 & LCOGT & SNIa & 0.015140 & -5 \\
\hline SN 2013hu & $11: 28: 07.2$ & $-13: 11: 24$ & C3776 & 20131228 & Itagaki & A5716 & 20140101 & PESSTO & SNII & 0.015000 & +25 \\
\hline SN 2013hx & $01: 35: 33.4$ & $-57: 57: 55$ & C3836 & 20131227 & SkyMapper & A5912 & 20140220 & PESSTO & SLSN & 0.135000 & +0 \\
\hline SN 2014D & $12: 10: 37.1$ & $+18: 49: 50$ & C3778 & 20140101 & CRTS & A5742 & 20140109 & ASP & SNIa & 0.008179 & -2 \\
\hline SN 2014E & 12:03:31.9 & $+02: 02: 46$ & C3784 & 20140105 & PTF & A5741 & 20140106 & PTF & SNIa & 0.018906 & -3 \\
\hline SN 2014I & $05: 42: 20.1$ & $-25: 32: 34$ & C3791 & 20140117 & Parker & A5777 & 20140118 & AWSNAP & SNIa & 0.030014 & -1 \\
\hline SN 2014L & $12: 18: 48.3$ & $+14: 24: 55$ & C3795 & 20140126 & TNTS & C3795 & 20140127 & TNTS & SNIc & 0.008029 & +0 \\
\hline SN 2014N & 02:30:59.0 & $-44: 25: 26$ & C3798 & 20140127 & Parker & C3798 & 20140202 & Drout & SNII & 0.017000 & +35 \\
\hline SN 2014X & $12: 10: 41.3$ & $-34: 03: 28$ & C3820 & 20140301 & Parker & A5948 & 20140304 & AWSNAP & SNIa & 0.021000 & +21 \\
\hline SN 2014aa & $11: 45: 03.3$ & $+19: 58: 38$ & C3823 & 20140307 & Arbor & A5958 & 20140309 & ASP & SNIa & 0.016982 & -7 \\
\hline SN 2014ac & $15: 04: 39.7$ & $+12: 38: 02$ & C3830 & 20140310 & ISSP & A5970 & 20140310 & PESSTO & SNIa & 0.030000 & +0 \\
\hline SN 2014ad & $11: 57: 44.0$ & $-10: 10: 03$ & C3831 & 20140312 & CRTS & C3831 & 20140315 & Jha & SNIc-BL & 0.005700 & (young \\
\hline SN 2014ah & $11: 55: 30.4$ & $+11: 55: 28$ & C3835 & 20140312 & ISSP & C3835 & 20140311 & ASP & SNIa & 0.021026 & -7 \\
\hline SN 2014ao & $08: 34: 33.2$ & $-02: 32: 33$ & C3855 & 20140417 & LOSS & A6077 & 20140417 & ASP & SNIa & 0.014000 & -10 \\
\hline SN 2014ap & 11:30:13.3 & $+24: 10: 17$ & C3856 & 20140320 & ISSP & A6001 & 20140322 & AWSNAP & SNIa & 0.023516 & -1 \\
\hline SN 2014at & 21:46:13.9 & $-46: 31: 21$ & C3862 & 20140420 & Parker & A6087 & 20140420 & PESSTO & SNIa & 0.030000 & -7 \\
\hline SN 2014au & $14: 19: 29.6$ & $-05: 37: 29$ & C3864 & 20140420 & CRTS & A6091 & 20140422 & PESSTO & SNII & 0.034920 & +16 \\
\hline SN 2014bx & $19: 43: 52.4$ & $-70: 37: 52$ & C3926 & 20140720 & Parker & A6335 & 20140722 & AWSNAP & SNIa & 0.011570 & -1 \\
\hline SN 2014bz & $13: 56: 04.2$ & $-43: 35: 10$ & C3932 & 20140506 & Conseil & A6230 & 20140611 & AWSNAP & SNIa & 0.022516 & +30 \\
\hline SN 2014ca & $16: 53: 20.9$ & $-72: 30: 52$ & C3935 & 20140510 & Parker & A6230 & 20140611 & AWSNAP & SNII & 0.019487 & +65 \\
\hline SN $2014 \mathrm{~cm}$ & 18:08:30.6 & $-52: 04: 11$ & C3951 & 20140530 & Parker & A6230 & 20140611 & AWSNAP & SNII & 0.018219 & +71 \\
\hline SN 2014co & $01: 10: 35.5$ & $-30: 13: 33$ & C3953 & 20140621 & Monard & A6302 & 20140708 & AWSNAP & SNII & 0.019036 & +10 \\
\hline SN 2014cp & $02: 25: 29.7$ & $-25: 37: 36$ & C3954 & 20140623 & Parker & A6302 & 20140708 & AWSNAP & SNIc-BL & 0.016164 & +1 \\
\hline SN 2014cq & 09:23:29.6 & $-63: 40: 28$ & C3955 & 20140620 & Parker & A6302 & 20140708 & AWSNAP & SNIIb & 0.010673 & +20 \\
\hline SN 2014cr & $19: 37: 05.5$ & $-42: 17: 44$ & C3956 & 20140627 & Monard & A6302 & 20140708 & AWSNAP & SNIax & 0.019123 & +23 \\
\hline SN 2014cs & $21: 35: 51.9$ & $-62: 05: 33$ & C3957 & 20140622 & Parker & A6302 & 20140708 & AWSNAP & SNIa & 0.053664 & +23 \\
\hline SN 2014ct & $09: 59: 25.2$ & $-00: 15: 19$ & C3958 & 20140601 & Parker & A6230 & 20140611 & AWSNAP & SNIa & 0.047987 & +24 \\
\hline SN 2014cu & 21:50:47.1 & $-73: 55: 27$ & C3959 & 20140622 & Parker & A6302 & 20140708 & AWSNAP & SNII & 0.028326 & +20 \\
\hline SN 2014cw & $22: 15: 26.7$ & $-10: 28: 40$ & C3962 & 20140829 & MASTER & A6440 & 20140902 & PESSTO & SNII & 0.006000 & +0 \\
\hline SN 2014cx & 00:59:47.9 & $-07: 34: 26$ & C3963 & 20140902 & Itagaki & A6440 & 20140902 & PESSTO & SNII & 0.005490 & (young) \\
\hline SN 2014cy & $23: 44: 16.1$ & $+10: 46: 05$ & C3964 & 20140831 & Nishimura & A6437 & 20140902 & LCOGT & SNII & 0.005547 & (young \\
\hline SN 2014dc & $03: 30: 54.6$ & $-47: 58: 49$ & C3970 & 20140904 & Maza & C3970 & 20140910 & Beletsky/AWSNAP & SNII & 0.022436 & -2 \\
\hline SN 2014df & $03: 44: 24.5$ & $-44: 40: 04$ & C3977 & 20140603 & Monard & A6201 & 20140603 & AWSNAP & SNIb & 0.003896 & +6 \\
\hline SN 2014dl & $16: 29: 46.1$ & $+08: 38: 31$ & C3995 & 20140925 & CRTS & A6507 & 20140926 & ASP & SNIa-91T & 0.032966 & -6 \\
\hline SN 2014dn & $04: 17: 54.8$ & $-56: 36: 48$ & C3997 & 20140924 & Parker & A6510 & 20140928 & Morrell & SNIa-pec & 0.022189 & +3 \\
\hline SN 2014do & 08:17:47.8 & $-30: 07: 25$ & $\mathrm{C} 4000$ & 20140928 & Parker & A6536 & 20141003 & AWSNAP & SNII & 0.005520 & +13 \\
\hline SN 2014dp & 08:19:38.2 & $-22: 34: 17$ & $\mathrm{C} 4002$ & 20140928 & Parker & A6562 & 20141009 & AWSNAP & $\mathrm{SNIb}$ & 0.018951 & +11 \\
\hline SN 2014dq & $22: 23: 16.4$ & $-28: 58: 34$ & A6592 & 20141019 & ASAS-SN & A6600 & 20141020 & Challis & SNII & 0.006000 & (young \\
\hline SN 2014dr & $03: 22: 46.6$ & $+00: 09: 28$ & C4009 & 20141014 & CRTS & A6610 & 20141023 & ASP & SNII & 0.023000 & +45 \\
\hline SN 2014eg & 02:45:09.0 & $-55: 44: 27$ & A6711 & 20141116 & PESSTO & A6739 & 20141122 & PESSTO & SNIa-91T & 0.018000 & -7 \\
\hline SN 2015F & $07: 36: 16.3$ & $-69: 30: 17$ & C4081 & 20150309 & Monard & A7209 & 20150310 & PESSTO & SNIa & 0.004000 & -11 \\
\hline SN 2015J & 07:35:07.0 & $-69: 08: 01$ & C4115 & 20150427 & SkyMapper & A7711 & 20150624 & AWSNAP & SNIIn & 0.005400 & (unk) \\
\hline SN 2015L & $22: 02: 14.3$ & $-61: 39: 32$ & A7642 & 20150614 & ASAS-SN & A7774 & 20150621 & Dong & SLSN & 0.232600 & (unk) \\
\hline SN 2015S & $00: 31: 26.7$ & $-49: 35: 35$ & C4130 & 20150722 & Marples & A7866 & 20150731 & AWSNAP & SNII & 0.021912 & +2 \\
\hline
\end{tabular}


Table A1. Continued.

\begin{tabular}{|c|c|c|c|c|c|c|c|c|c|c|c|}
\hline \multirow[b]{2}{*}{$\mathrm{SN}$} & \multicolumn{5}{|c|}{ Discovery } & \multicolumn{6}{|c|}{ Classification } \\
\hline & RA & DEC & $\operatorname{Ref}^{a}$ & Date & Group $^{b}$ & Ref. & Date & Group $^{c}$ & SN type & Redshift & Phase $^{d}$ \\
\hline ASASSN-13co & $21: 40: 38.5$ & $+06: 30: 40$ & A5346 & 20130829 & ASAS-SN & A5353 & 20130901 & Morrell & SNII & 0.022600 & +0 \\
\hline ASASSN-14dp & $11: 21: 58.4$ & $-37: 54: 25$ & A6297 & 20140707 & ASAS-SN & A6302 & 20140708 & AWSNAP & SNII & 0.009159 & -1 \\
\hline ASASSN-14eu & $15: 00: 36.9$ & $-03: 50: 52$ & A6360 & 20140801 & ASAS-SN & A6363 & 20140802 & Milisavljevic & SNIa & 0.022722 & +0 \\
\hline ASASSN-14ew & 20:22:00.7 & $-51: 47: 48$ & A6367 & 20140804 & ASAS-SN & A6384 & 20140811 & Takats & SNIa & 0.019597 & +0 \\
\hline ASASSN-14fo & $20: 34: 06.5$ & $-01: 58: 13$ & A6397 & 20140815 & ASAS-SN & A6399 & 20140816 & PESSTO & SNIa & 0.020000 & +2 \\
\hline ASASSN-14fw & $23: 49: 06.7$ & $-07: 03: 05$ & A6404 & 20140818 & ASAS-SN & A6417 & 20140824 & PESSTO & SNIa & 0.019000 & +10 \\
\hline ASASSN-14ha & 04:20:02.0 & $-54: 56: 19$ & A6460 & 20140910 & ASAS-SN & A6466 & 20140913 & LCOGT & SNII & 0.005017 & (young \\
\hline ASASSN-14hr & 01:50:41.6 & $-14: 31: 08$ & $\mathrm{~A} 6500$ & 20140925 & ASAS-SN & A6508 & 20140927 & CSP & SNIa & 0.033620 & -5 \\
\hline ASASSN-14hu & $06: 43: 27.5$ & $-69: 38: 17$ & A6505 & 20140927 & ASAS-SN & A6510 & 20140928 & CSP & SNIa & 0.015000 & -5 \\
\hline ASASSN-14ig & 01:16:05.3 & $-61: 37: 21$ & A6517 & 20140921 & ASAS-SN & A6536 & 20141003 & AWSNAP & SNIa & 0.030000 & +15 \\
\hline ASASSN-14ih & $22: 34: 30.4$ & $-24: 39: 54$ & A6521 & 20140919 & ASAS-SN & A6536 & 20141003 & AWSNAP & SNIa & 0.032000 & +20 \\
\hline ASASSN-14ii & $22: 16: 41.5$ & $-36: 09: 42$ & A6521 & 20140922 & ASAS-SN & A6536 & 20141003 & AWSNAP & SNIa & 0.030000 & +9 \\
\hline ASASSN-14il & $00: 45: 33.0$ & $-14: 15: 34$ & A6525 & 20141001 & ASAS-SN & A6536 & 20141003 & AWSNAP & SNIIn & 0.022000 & (young \\
\hline ASASSN-14jc & $07: 35: 34.7$ & $-62: 46: 10$ & A6594 & 20141019 & ASAS-SN & A6618 & 20141023 & PESSTO & SNIa & 0.011320 & +0 \\
\hline ASASSN-14jg & $23: 33: 14.5$ & $-60: 34: 13$ & A6637 & 20141021 & ASAS-SN & A6661 & 20141102 & LCOGT & SNIa & 0.014800 & +0 \\
\hline ASASSN-14ko & $05: 25: 16.5$ & $-45: 59: 24$ & A6732 & 20141114 & ASAS-SN & A6749 & 20141124 & PESSTO & SNIIn & 0.042000 & (unk) \\
\hline ASASSN-14kp & $00: 39: 38.6$ & $-38: 04: 38$ & A6736 & 20141122 & ASAS-SN & A6765 & 20141127 & CSP & SNII & 0.023000 & +0 \\
\hline ASASSN-14kq & $23: 45: 15.5$ & $-29: 47: 01$ & A6737 & 20141123 & ASAS-SN & A6765 & 20141127 & CSP & SNIa & 0.034000 & -2 \\
\hline ASASSN-14lp & $12: 45: 08.6$ & $-00: 27: 29$ & A6795 & 20141210 & ASAS-SN & A6801 & 20141211 & Thorstensen & SNIa & 0.005101 & -3 \\
\hline ASASSN-14lt & 03:11:07.4 & $-13: 06: 30$ & A6802 & 20141210 & ASAS-SN & A6804 & 20141212 & ASP & SNIa & 0.032000 & -7 \\
\hline ASASSN-14lw & 01:06:47.6 & $-46: 58: 55$ & A6809 & 20141212 & ASAS-SN & A6832 & 20141216 & AWSNAP & SNIa-91T & 0.016000 & -4 \\
\hline ASASSN-15aj & $10: 52: 54.2$ & $-32: 55: 26$ & A6886 & 20150108 & ASAS-SN & A6905 & 20150112 & Simon & SNIa & 0.010921 & +1 \\
\hline ASASSN-15al & $04: 57: 49.6$ & $-21: 35: 35$ & A6894 & 20150104 & ASAS-SN & A6909 & 20150112 & PESSTO & SNIa & 0.030000 & +0 \\
\hline ASASSN-15as & 09:39:16.8 & $+06: 25: 56$ & A6919 & 20150113 & ASAS-SN & A6920 & 20150114 & Morrell & SNIa & 0.030000 & +0 \\
\hline ASASSN-15az & $11: 08: 38.0$ & $-10: 14: 48$ & A6921 & 20150114 & ASAS-SN & A6925 & 20150115 & ASP & SNIa & 0.028000 & +0 \\
\hline ASASSN-15ba & 14:04:55.1 & $+08: 55: 15$ & A6934 & 20150115 & ASAS-SN & A6947 & 20150115 & Challis & SNIa & 0.023100 & -5 \\
\hline ASASSN-15dz & $16: 41: 20.9$ & $+08: 54: 32$ & A7145 & 20150221 & ASAS-SN & A7180 & 20150306 & AWSNAP & SNIa & 0.032216 & +9 \\
\hline ASASSN-15eb & 08:06:07.8 & $-22: 33: 51$ & A7157 & 20150226 & ASAS-SN & A7180 & 20150306 & AWSNAP & SNIa & 0.016481 & +8 \\
\hline ASASSN-15go & $06: 11: 22.5$ & $-16: 25: 21$ & A7349 & 20150406 & ASAS-SN & A7368 & 20150411 & AWSNAP & SNIa & 0.018923 & -4 \\
\hline ASASSN-15hs & $15: 33: 34.7$ & $-78: 07: 12$ & A7444 & 20150424 & ASAS-SN & A7458 & 20150429 & AWSNAP & SNIIn & 0.008900 & (unk) \\
\hline ASASSN-15hx & $13: 43: 16.7$ & $-31: 33: 21$ & A7447 & 20150426 & ASAS-SN & A7452 & 20150426 & PESSTO & SNIa & 0.010000 & -11 \\
\hline ASASSN-15hy & 20:10:01.6 & $-00: 44: 16$ & A7450 & 20150425 & ASAS-SN & A7452 & 20150426 & PESSTO & SNIa-pec & 0.025000 & -13 \\
\hline ASASSN-15hz & $22: 29: 37.8$ & $-65: 27: 44$ & A7450 & 20150426 & ASAS-SN & A7458 & 20150429 & AWSNAP & SNIa & 0.033000 & -3 \\
\hline ASASSN-15ic & $06: 14: 52.0$ & $-42: 48: 00$ & A7455 & 20150427 & ASAS-SN & A7458 & 20150429 & AWSNAP & SNIa & 0.025000 & -1 \\
\hline ASASSN-15lv & 01:59:01.0 & $-32: 22: 28$ & A7700 & 20150705 & ASAS-SN & A7746 & 20150701 & AWSNAP & SNIIb & 0.011000 & -2 \\
\hline SNhunt222 & 10:00:26.2 & $+03: 22: 50$ & СТОСР & 20131214 & CRTS/LSQ & A5860 & 20140207 & PESSTO & SNII & 0.006860 & +60 \\
\hline SNhunt239 & 12:09:09.7 & $+31: 34: 28$ & СТОСР & 20140325 & CRTS & A6040 & 20140402 & AWSNAP & SNIa-91bg & 0.022666 & +10 \\
\hline SNhunt259 & 00:00:21.6 & $-02: 36: 23$ & СТОСР & 20141015 & CRTS & (NEW) & 20141018 & AWSNAP & SNIa & 0.037909 & +100 \\
\hline SNhunt261 & $02: 45: 29.8$ & $-17: 31: 41$ & СТОСР & 20141017 & CRTS & A6629 & 20141027 & AWSNAP & SNIa-91bg & 0.029070 & +2 \\
\hline CSS1222 & $12: 22: 57.0$ & $+28: 29: 57$ & CRTS & 20140326 & CRTS & A6040 & 20140402 & AWSNAP & SNII & 0.024000 & +2 \\
\hline Gaia15agm & $23: 55: 57.6$ & $-43: 43: 40$ & Gaia & 20150601 & Gaia & A7746 & 20150701 & AWSNAP & SNIa & 0.029000 & +31 \\
\hline LSQ12dlf & 01:50:29.8 & $-21: 48: 45$ & LSQ & 20120710 & LSQ & A4329 & 20120818 & PESSTO & SLSN-Ic & 0.250000 & +21 \\
\hline LSQ12ege & $16: 50: 51.7$ & $+02: 52: 40$ & LSQ & 20120715 & LSQ & A4303 & 20120809 & PESSTO & SNIa & 0.080000 & -5 \\
\hline LSQ12ehh & $16: 27: 12.4$ & $+02: 05: 12$ & LSQ & 20120727 & LSQ & A4303 & 20120809 & PESSTO & SNIa & 0.100000 & -6 \\
\hline LSQ12ers & 23:02:03.3 & $-22: 52: 17$ & LSQ & 20120829 & LSQ & A4357 & 20120906 & AWSNAP & SNIa & 0.099000 & +1 \\
\hline
\end{tabular}


Table A1. Continued.

\begin{tabular}{|c|c|c|c|c|c|c|c|c|c|c|c|}
\hline \multirow[b]{2}{*}{$\mathrm{SN}$} & \multicolumn{5}{|c|}{ Discovery } & \multicolumn{6}{|c|}{ Classification } \\
\hline & RA & DEC & $\operatorname{Ref}^{a}$ & Date & Group $^{b}$ & Ref. & Date & Group $^{c}$ & SN type & Redshift & Phase $^{d}$ \\
\hline LSQ12euu & $21: 31: 07.5$ & $-06: 41: 49$ & LSQ & 20120902 & LSQ & A4357 & 20120906 & AWSNAP & SNIa & 0.116000 & -1 \\
\hline LSQ12eve & $21: 53: 16.7$ & $-21: 42: 45$ & LSQ & 20120903 & LSQ & A4357 & 20120906 & AWSNAP & SNIa & 0.098000 & -7 \\
\hline LSQ12gdj & $23: 54: 45.3$ & $-25: 40: 36$ & LSQ & 20121107 & LSQ & A4566 & 20121110 & SNF & SNIa-91T & 0.030324 & -5 \\
\hline LSQ13ddu & 03:58:48.4 & $-29: 24: 54$ & LSQ & 20131122 & LSQ & - & 20131130 & PESSTO & SLSN & 0.058000 & (unk) \\
\hline LSQ13pp & $12: 42: 02.9$ & $-22: 05: 07$ & LSQ & 20130318 & LSQ & (NEW) & 20130325 & AWSNAP & SNII & 0.045700 & +7 \\
\hline LSQ14bcj & $14: 05: 58.2$ & $-12: 04: 12$ & LSQ & 20140403 & LSQ & A6087 & 20140420 & PESSTO & SNIa & 0.020000 & -7 \\
\hline LSQ15adm & $20: 14: 20.9$ & $-58: 08: 01$ & LSQ & 20150405 & LSQ & A7363 & 20150409 & PESSTO & SNIa-csm & 0.073000 & +10 \\
\hline LSQ15ey & 14:12:05.1 & $-07: 23: 37$ & LSQ & 20150120 & LSQ & A6994 & 20150125 & PESSTO & SNII & 0.036000 & (young \\
\hline MASTERJ1408 & 14:08:04.9 & $-11: 59: 46$ & A6084 & 20140330 & MASTER & A6092 & 20140423 & AWSNAP & SNIa & 0.035308 & +25 \\
\hline MASTERJ1353 & $13: 53: 30.3$ & $-42: 16: 19$ & A7151 & 20150227 & MASTER & A7209 & 20150310 & PESSTO & SNIIn & 0.052000 & +10 \\
\hline MASTERJ0746 & 07:46:10.7 & $-71: 22: 20$ & A7451 & 20150426 & MASTER & A7458 & 20150429 & AWSNAP & SNIa & 0.017400 & -7 \\
\hline OGLE-2013-SN-070 & $00: 45: 15.0$ & $-66: 19: 06$ & A5397 & 20130910 & OGLE & A5455 & 20131008 & AWSNAP & SNIa & 0.043000 & +33 \\
\hline OGLE-2014-SN-019 & 06:13:50.4 & $-67: 55: 13$ & A5916 & 20140222 & OGLE & A5915 & 20140222 & PESSTO & SNIa & 0.040000 & -3 \\
\hline OGLE-2014-SN-021 & $05: 48: 25.3$ & $-66: 47: 31$ & A5916 & 20140224 & OGLE & A5919 & 20140224 & AWSNAP & SNIa & 0.039000 & +0 \\
\hline OGLE-2014-SN-067 & 04:57:50.4 & $-74: 00: 40$ & A6423 & 20140815 & OGLE & A6430 & 20140829 & AWSNAP & SNIc & 0.018700 & +0 \\
\hline PS1-14lz & 04:50:48.8 & $-14: 25: 23$ & PS1 & 20140213 & PanSTARRS & A5908 & 20140219 & PESSTO & SNIa & 0.070000 & +1 \\
\hline PS1-14md & $11: 17: 42.3$ & $-21: 14: 35$ & PS1 & 20140213 & PanSTARRS & A5919 & 20140224 & AWSNAP & SNIa & 0.071000 & +3 \\
\hline PS1-14ra & $14: 41: 28.3$ & $+09: 26: 12$ & PS1 & 20140305 & PanSTARRS & A5957 & 20140307 & PESSTO & SNIa & 0.030000 & +0 \\
\hline PS1-14vw & 09:58:07.5 & $+07: 57: 57$ & PS1 & 20140325 & PanSTARRS & A6040 & 20140402 & AWSNAP & SNIa & 0.091000 & +14 \\
\hline PS1-14vx & $10: 41: 50.9$ & $+16: 49: 06$ & PS1 & 20140325 & PanSTARRS & A6040 & 20140402 & AWSNAP & SNIa & 0.057000 & +23 \\
\hline PS1-14wl & $13: 32: 42.0$ & $-20: 36: 24$ & PS1 & 20140325 & PanSTARRS & A6040 & 20140402 & AWSNAP & SNIa & 0.034000 & +0 \\
\hline PS15ae & $11: 33: 41.7$ & $+00: 43: 40$ & PS1 & 20141223 & CRTS/PS1 & A7102 & 20150217 & PESSTO & SLSN-Ic & 0.110000 & +0 \\
\hline PS15br & $11: 25: 19.2$ & $+08: 14: 18$ & PS1 & 20150215 & PanSTARRS & A7209 & 20150310 & PESSTO & SLSN & 0.101000 & (unk) \\
\hline
\end{tabular}


Table A1. Continued.

\begin{tabular}{|c|c|c|c|c|c|c|c|c|c|c|c|}
\hline \multirow[b]{2}{*}{ SN } & \multicolumn{5}{|c|}{ Discovery } & \multicolumn{6}{|c|}{ Classification } \\
\hline & RA & DEC & Ref. $^{a}$ & Date & Group $^{b}$ & Ref. & Date & Group $^{c}$ & SN type & Redshift & Phase $^{d}$ \\
\hline PSNJ02454138-0812301 & $02: 45: 41.4$ & $-08: 12: 30$ & СТОСР & 20141024 & ISSP & A6628 & 20141027 & ASP & SNIa & 0.029613 & +3 \\
\hline PSNJ05031639-0256110 & 05:03:16.4 & $-02: 56: 11$ & СТОСР & 20141105 & LOSS & A6682 & 20141110 & Kangas & SNIb & 0.014440 & +0 \\
\hline PSNJ09023787+2556042 & 09:02:37.9 & $+25: 56: 04$ & A7366 & 20150411 & LOSS & A7368 & 20150411 & AWSNAP & SNIIb & 0.008172 & -3 \\
\hline PSNJ09204691-0803340 & 09:20:46.9 & $-08: 03: 34$ & СТОСР & 20141120 & Gagliano & A6734 & 20141121 & NAOC & SNIIn & 0.019644 & -10 \\
\hline PSNJ10433393-3048206 & $10: 43: 33.9$ & $-30: 48: 21$ & СTOCP & 20150211 & Parker & A7073 & 20150212 & Morrell & SNIIn & 0.013000 & +117 \\
\hline PSNJ11484578-2817312 & $11: 48: 45.8$ & $-28: 17: 31$ & СТOCP & 20131127 & CHASE & A5615 & 20131129 & PESSTO & SNIc-BL & 0.010000 & +20 \\
\hline PSNJ13471211-2422171 & $13: 47: 12.1$ & $-24: 22: 17$ & СТOCP & 20150212 & Parker & A7075 & 20150215 & Stritzinger & SNIa & 0.019910 & +0 \\
\hline PSNJ17194328-7721305 & $17: 19: 43.3$ & $-77: 21: 31$ & СТОСР & 20130825 & Aldous & A5331 & 20130826 & PESSTO & SNIa & 0.009000 & -4 \\
\hline PSNJ17285509-6609103 & $17: 28: 55.1$ & $-66: 09: 10$ & СТОСР & 20130827 & Marples & A5338 & 20130828 & PESSTO & SNII & 0.021908 & +7 \\
\hline PSNJ19251269-5336028 & $19: 25: 12.7$ & $-53: 36: 03$ & СТОСР & 20150810 & Parker & A7925 & 20150817 & AWSNAP & SNIa & 0.054000 & +48 \\
\hline PSNJ20250386-2449133 & $20: 25: 04.1$ & $-24: 49: 20$ & A6659 & 20141028 & LOSS & A6667 & 20141104 & LCOGT & SNIc & 0.010614 & -3 \\
\hline PSNJ23320218-4545032 & $23: 32: 02.2$ & $-45: 45: 03$ & СТОСР & 20120828 & CRTS & (NEW) & 20120907 & AWSNAP & SNIc & 0.005000 & +60 \\
\hline PTF12gzk & $22: 12: 41.5$ & $+00: 30: 53$ & A4298 & 20120724 & PTF & A4298 & 20120727 & PTF & SNIc-BL & 0.013700 & (young) \\
\hline iPTF13bvn & $14: 59: 59.4$ & $+01: 52: 59$ & A5147 & 20130616 & iPTF & A5142 & 20140617 & Milisavljevic & SNIb & 0.004490 & -32 \\
\hline iPTF13dge & $05: 03: 35.3$ & $+01: 34: 15$ & A5366 & 20130904 & iPTF & A5366 & 20130904 & PTF & SNIa & 0.015854 & -15 \\
\hline SMTJ10310056-3658262 & $10: 30: 40.0$ & $-36: 57: 51$ & A7261 & 20150313 & SkyMapper & A7261 & 20150319 & PESSTO & SNIa & 0.035000 & +0 \\
\hline SMTJ03253351-5344190 & $03: 25: 33.8$ & $-53: 44: 22$ & A5641 & 20131201 & SkyMapper & A5641 & 20131206 & AWSNAP & SNIa & 0.055000 & -6 \\
\hline SMTJ05451320-4735425 & $05: 45: 13.7$ & $-47: 35: 37$ & - & 20150315 & SkyMapper & - & - & LCOGT & SNIa & 0.050000 & +0 \\
\hline SMTJ13494831-0658174 & $13: 49: 48.0$ & $-06: 58: 17$ & A7925 & 20150728 & SkyMapper & A7925 & 20150817 & AWSNAP & SNIa & 0.076933 & +12 \\
\hline
\end{tabular}

a Notation: A1234 $\equiv$ ATel \# 1234; C1234 $\equiv$ CBET \# 1234; CTOCP $\equiv$ CBET Transient Object Confirmation Page; objects newly classified here denoted as (NEW); all others refer to transient announcement webpages for stated discovery group(s).

${ }^{b}$ Discovery group acronyms (where appropriate— see text for details) or lead author of the discovery alert.

${ }^{c}$ Classifcation group acronyms (where appropriate - see text for details) or lead author of the classification alert.

${ }^{d}$ Phase with respect to maximum light (where appropriate) for the best spectroscopic match, as reported by the classifying group. SNe IIn do not typically have a clearly identifiable spectroscopic phase so are listed as '(unk)' (i.e. unknown). Very young SNe II (with very blue featureless continua) similarly have ambiguous phases so are listed as '(young)'. 
DR1 data release. We list the discovery location and date, as well as discovery reference and group that discovered the transient. Note the discovery references are typically either Astronomer's Telegrams (ATel's, denoted as, e.g. 'A1234') or telegrams from the Central Bureau for Electronic Telegrams (CBET's, denoted as, e.g. 'C1234'), though occasionally a separate discovery notice was not issuedin these cases, typically the webpage of the discovering group was cited in the classification reference. Classification references are given with similar notation, listed along with classification date, quoted supernova type and phase from the classification, as well as the redshift listed in the classification.

In Table A1, the discovery and classification groups are listed either as acronyms of the group name, or listed as the first author in the discovery/classification notice. The acronyms used in the table for discovery groups are as follows:

- ASAS-SN: the All-Sky Automated Survey for Supernovae (Shappee et al. 2014).

- CHASE: the CHilean Automatic Supernova sEarch (Pignata et al. 2009).

- CRTS: the Catalina Real-Time Transient Survey (Drake et al. 2009).

- Gaia: transient alerts from Gaia (e.g. Wyrzykowski et al. 2012; Blagorodnova et al. 2014, 2016).

- ISSP: the Italian Supernova Search Programme.

- LOSS: the Lick Observatory Supernova Search (Ganeshalingam et al. 2010).

- LSQ: the La Silla-QUEST low redshift supernova survey (Baltay et al. 2013).

- MASTER: the Mobile Astronomical System of TelescopeRobots (Lipunov et al. 2004).

- OGLE: the OGLE-IV real-time transient search (Wyrzykowski et al. 2014).

- PanSTARRS: the Panoramic Survey Telescope and Rapid Response System panstarrs.

- PTF/iPTF: the Palomar Transient Factory (Rau et al. 2009; Law et al. 2009) and its successor.

- SkyMapper: the SkyMapper (Keller et al. 2007) transients search.

- TAROT: the Télescope á Action Rapide pour les Objets Transitoires (Rapid Action Telescope for Transient Objects) at La Silla (e.g. Klotz et al. 2013).

- TNTS: the Tsinghua-NAOC Transient Survey.

The acronyms used in the table for classification groups are as follows:

- ASP: the Asiago Supernova Programme (Tomasella et al. 2014).

- AWSNAP: the ANU+WiFeS SuperNovA Programme (this work).

- CSP: the Carnegie Supernova Project (Folatelli et al. 2013).

- LCOGT: the Las Cumbres Observatory Global Telescope (Brown et al. 2013).

- PESSTO: the Public ESO Spectroscopic Survey for Transient Objects (Smartt et al. 2015).

- SNF: SuperNova Factory (Aldering et al. 2002).

Some transients in Table A1 are known by other aliases or are listed in shorthand form (due to a lengthy full name). These transients and their aliases (or full names) are noted in Table A2.
Table A2. Alternate and/or full designations for $\mathrm{SNe}$ in the AWSNAP sample.

\begin{tabular}{ll}
\hline \hline SN & \multicolumn{1}{c}{ Alias } \\
\hline SN 2013ef & ASASSN-13bb \\
SN 2014E & PTF14w \\
SN 2014cx & ASASSN-14gm \\
SN 2014dq & ASASSN-14jb \\
SN 2015L & ASASSN-15lh \\
iPTF13dge & PS1-13dvn \\
LSQ15adm & Gaia15aep \\
LSQ15ey & PS15ii \\
PS15ae & CSS141223:113342+004332 \\
PSNJ09023787+2556042 & Gaia15aet \\
PSNJ10433393-3048206 & PS15ip \\
PSNJ20250386-2449133 & PS15bjv \\
SNhunt222 & LSQ14rl \\
CSS1222 & PS1-14ya \\
CSS1222 & CSS140326:122257+282955 \\
MASTERJ1408 & MASTER OT J140804.26-115949.7 \\
MASTERJ1353 & MASTER OT J135329.90-421622.5 \\
MASTERJ0746 & MASTER OT J074610.09-712224.9 \\
\hline
\end{tabular}

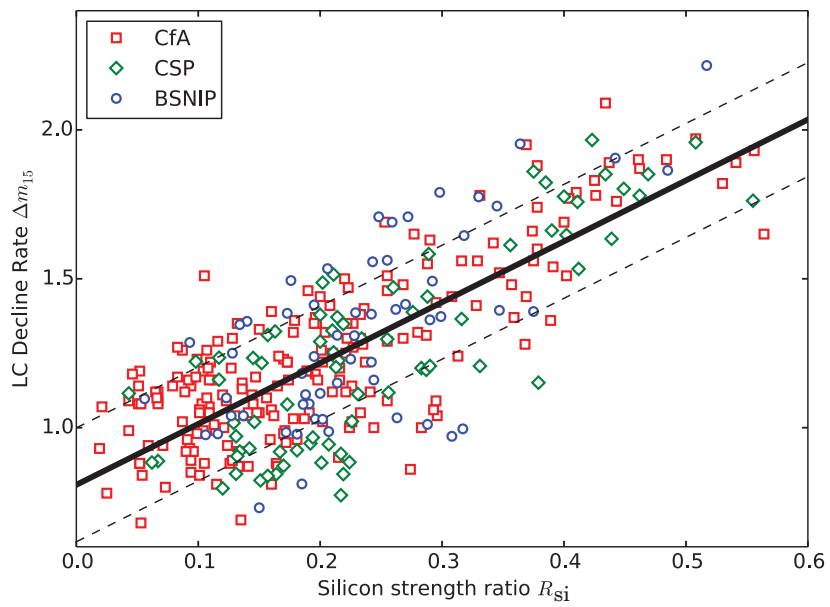

Figure A1. Literature data used to fit the trend of $\Delta m_{15}$ versus $R_{\mathrm{Si}}$. The best fit is the thick solid line, and the thin dashed line represent the trend $\pm 1 \sigma$ (where $\sigma$ is the dispersion of the data about the trend).

AWSNAP spectra: The full list of AWSNAP spectra released here is given in Table A3, along with pertinent observation details such as grating and exposure time.

SN Ia sodium fits: In Table A4, we present the best fit parameters (and uncertainties) for our fits to narrow sodium absorption in our sample of SN Ia maximum light spectra. Table A5 lists the redshifts used to establish the local rest velocity of the $\mathrm{SN}$-typically this was the redshift of the host galaxy but occasionally is obtained from galaxy emission features at the location of the SN (denoted as 'Local').

\section{APPENDIX A2: DERIVATION OF THE SN Ia $R_{\mathrm{Si}}-\Delta m_{15}$ RELATION FROM LITERATURE DATA}

Our fit to the relation between $\Delta m_{15}$ and $R_{\mathrm{Si}}$ is presented here. Our fit to the data is shown in Figure A1, and from these data we derive 
Table A3. All AWSNAP spectra.

\begin{tabular}{|c|c|c|c|}
\hline $\mathrm{SN}$ & $\begin{array}{c}\text { Observation } \\
\text { date }\end{array}$ & Grating & $\begin{array}{c}\text { Exposure } \\
\text { time (s) }\end{array}$ \\
\hline SN 2009ip & 2012-Oct-22 & B3000 & 1200 \\
\hline SN 2009ip & 2012-Oct-22 & R3000 & 1200 \\
\hline SN 2009ip & 2012-Oct-23 & B7000 & 1200 \\
\hline SN 2009ip & 2012-Oct-23 & R7000 & 1200 \\
\hline SN 2009ip & 2012-Sep-22 & B3000 & 1200 \\
\hline SN 2009ip & 2012-Sep-22 & R3000 & 1200 \\
\hline SN 2009ip & 2012-Sep-23 & B3000 & 1200 \\
\hline SN 2009ip & 2012-Sep-23 & R3000 & 1200 \\
\hline SN 2009ip & 2013-Apr-12 & B7000 & 1200 \\
\hline SN 2009ip & 2013-Apr-12 & R7000 & 1200 \\
\hline SN 2009ip & 2013-Jun-22 & B7000 & 1200 \\
\hline SN 2009ip & 2013-Jun-22 & R7000 & 1200 \\
\hline SN 2009ip & 2013-May-26 & B3000 & 1200 \\
\hline SN 2009ip & 2013-May-26 & R7000 & 1200 \\
\hline SN 2012ca & 2012-Aug-10 & В3000 & 1200 \\
\hline SN 2012ca & 2012-Aug-10 & R3000 & 1200 \\
\hline SN 2012ca & 2012-Sep-06 & B3000 & 1200 \\
\hline SN 2012ca & 2012-Sep-06 & R3000 & 1200 \\
\hline SN 2012ca & 2012-Sep-07 & B3000 & 1200 \\
\hline SN 2012ca & 2012-Sep-07 & R3000 & 1200 \\
\hline SN 2012ca & 2012-Sep-22 & B3000 & 1200 \\
\hline SN 2012ca & 2012-Sep-22 & R3000 & 1200 \\
\hline SN 2012ca & 2012-Sep-23 & B3000 & 1200 \\
\hline SN 2012ca & 2012-Sep-23 & R3000 & 1200 \\
\hline SN 2012ca & 2013-Jun-03 & B3000 & 1200 \\
\hline SN 2012ca & 2013-Jun-03 & R7000 & 1200 \\
\hline SN 2012ca & 2013-Мay-26 & В3000 & 1200 \\
\hline SN 2012ca & 2013-Мay-26 & R3000 & 1200 \\
\hline SN 2012ca & 2013-Мay-26 & R7000 & 1200 \\
\hline SN 2012dj & 2012-Aug-11 & В3000 & 1200 \\
\hline SN 2012dj & 2012-Aug-11 & R3000 & 1200 \\
\hline SN 2012dj & 2012-Aug-12 & B3000 & 1200 \\
\hline SN 2012dj & 2012-Aug-12 & R3000 & 1200 \\
\hline SN 2012dj & 2012-Aug-13 & В3000 & 1200 \\
\hline SN 2012dj & 2012-Aug-13 & R3000 & 1200 \\
\hline SN 2012dj & 2012-Sep-06 & B3000 & 1200 \\
\hline SN 2012dj & 2012-Sep-06 & R3000 & 1200 \\
\hline SN 2012dj & 2012-Sep-07 & B3000 & 1200 \\
\hline SN 2012dj & 2012-Sep-07 & R3000 & 1200 \\
\hline SN 2012dj & 2012-Sep-22 & В3000 & 1200 \\
\hline SN 2012dj & 2012-Sep-22 & R3000 & 1200 \\
\hline SN 2012dj & 2012-Sep-23 & B3000 & 1200 \\
\hline SN 2012dj & 2012-Sep-23 & R3000 & 1200 \\
\hline SN $2012 d n$ & 2012-Aug-11 & B3000 & 1200 \\
\hline SN $2012 d n$ & 2012-Aug-11 & R3000 & 1200 \\
\hline SN 2012 dn & 2012-Aug-12 & B3000 & 1200 \\
\hline SN $2012 d n$ & 2012-Aug-12 & R3000 & 1200 \\
\hline SN 2012dn & 2012-Aug-13 & B3000 & 1200 \\
\hline SN 2012dn & 2012-Aug-13 & R3000 & 1200 \\
\hline SN 2012dn & 2012-Jul-18 & B3000 & 600 \\
\hline SN 2012 dn & 2012-Jul-18 & R3000 & 600 \\
\hline SN 2012 dn & 2012-Jul-19 & B3000 & 600 \\
\hline SN 2012dn & 2012-Jul-19 & R3000 & 600 \\
\hline SN 2012dn & 2012-Jul-20 & B3000 & 180 \\
\hline SN 2012dn & 2012-Jul-20 & R3000 & 180 \\
\hline SN 2012dn & 2012-Jul-21 & B3000 & 1200 \\
\hline SN 2012 dn & 2012-Jul-21 & R3000 & 1200 \\
\hline SN 2012 dn & 2012-Oct-23 & B3000 & 1200 \\
\hline SN 2012dn & 2012-Oct-23 & R3000 & 1200 \\
\hline SN 2012dn & 2012-Sep-06 & B3000 & 1200 \\
\hline SN 2012 dn & 2012-Sep-06 & R3000 & 1200 \\
\hline
\end{tabular}

Table A3. Continued.

\begin{tabular}{|c|c|c|c|}
\hline $\mathrm{SN}$ & $\begin{array}{l}\text { Observation } \\
\text { date }\end{array}$ & Grating & $\begin{array}{c}\text { Exposure } \\
\text { time (s) }\end{array}$ \\
\hline SN 2012dn & 2012-Sep-07 & B3000 & 1200 \\
\hline SN 2012dn & 2012-Sep-07 & R3000 & 1200 \\
\hline SN 2012dn & 2012-Sep-22 & B3000 & 1200 \\
\hline SN 2012dn & 2012-Sep-22 & R3000 & 1200 \\
\hline SN 2012dn & 2012-Sep-23 & B3000 & 1200 \\
\hline SN 2012dn & 2012-Sep-23 & R3000 & 1200 \\
\hline SN 2012dt & 2012-Sep-22 & B3000 & 1200 \\
\hline SN 2012dt & 2012-Sep-22 & R3000 & 1200 \\
\hline SN 2012dy & 2012-Aug-11 & В3000 & 1200 \\
\hline SN 2012dy & 2012-Aug-11 & R3000 & 1200 \\
\hline SN 2012dy & 2012-Aug-12 & B3000 & 1200 \\
\hline SN 2012dy & 2012-Aug-12 & R3000 & 1200 \\
\hline SN 2012dy & 2012-Aug-13 & B3000 & 1200 \\
\hline SN 2012dy & 2012-Aug-13 & R3000 & 1200 \\
\hline SN 2012dy & 2012-Sep-06 & B3000 & 1200 \\
\hline SN 2012dy & 2012-Sep-06 & R3000 & 1200 \\
\hline SN 2012dy & 2012-Sep-07 & B3000 & 1200 \\
\hline SN 2012dy & 2012-Sep-07 & R3000 & 1200 \\
\hline SN 2012dy & 2012-Sep-22 & B3000 & 1200 \\
\hline SN 2012dy & 2012-Sep-22 & R3000 & 1200 \\
\hline SN 2012dy & 2012-Sep-23 & B3000 & 1200 \\
\hline SN 2012dy & 2012-Sep-23 & R3000 & 1200 \\
\hline SN 2012ec & 2012-Aug-12 & B3000 & 1200 \\
\hline SN 2012ec & 2012-Aug-12 & R3000 & 1200 \\
\hline SN 2012ec & 2012-Aug-13 & B3000 & 1200 \\
\hline SN 2012ec & 2012-Aug-13 & R3000 & 1200 \\
\hline SN 2012ec & 2012-Oct-22 & B3000 & 1200 \\
\hline SN 2012ec & 2012-Oct-22 & R3000 & 1200 \\
\hline SN 2012ec & 2012-Sep-01 & B3000 & 600 \\
\hline SN 2012ec & 2012-Sep-01 & R3000 & 600 \\
\hline SN 2012ec & 2012-Sep-03 & B3000 & 700 \\
\hline SN 2012ec & 2012-Sep-03 & R3000 & 700 \\
\hline SN 2012ec & 2012-Sep-06 & B3000 & 1200 \\
\hline SN 2012ec & 2012-Sep-06 & R3000 & 1200 \\
\hline SN 2012ec & 2012-Sep-07 & B3000 & 1200 \\
\hline SN 2012ec & 2012-Sep-07 & R3000 & 1200 \\
\hline SN 2012ec & 2012-Sep-22 & B3000 & 1200 \\
\hline SN 2012ec & 2012-Sep-22 & R3000 & 1200 \\
\hline SN 2012ec & 2012-Sep-23 & B3000 & 1200 \\
\hline SN 2012ec & 2012-Sep-23 & R3000 & 1200 \\
\hline SN 2012eq & 2012-Sep-07 & B3000 & 1200 \\
\hline SN 2012eq & 2012-Sep-07 & R3000 & 1200 \\
\hline SN 2012eu & 2012-Sep-07 & B3000 & 1200 \\
\hline SN 2012eu & 2012-Sep-07 & R3000 & 1200 \\
\hline SN 2012hr & 2013-Dec-30 & B3000 & 1200 \\
\hline SN 2012hr & 2013-Dec-30 & R3000 & 1200 \\
\hline SN 2013K & 2013-May-26 & B3000 & 1200 \\
\hline SN 2013K & 2013-May-26 & R3000 & 1200 \\
\hline SN 2013aa & 2013-Apr-09 & B3000 & 1200 \\
\hline SN 2013aa & 2013-Apr-09 & R3000 & 1200 \\
\hline SN 2013aa & 2013-Mar-25 & B3000 & 1200 \\
\hline SN 2013aa & 2013-Mar-25 & R3000 & 1200 \\
\hline SN 2013aa & 2013-Sep-13 & B3000 & 1200 \\
\hline SN 2013aa & 2013-Sep-13 & R3000 & 1200 \\
\hline SN 2013aa & 2014-Jan-31 & B3000 & 1200 \\
\hline SN 2013aa & 2014-Jan-31 & R3000 & 1200 \\
\hline SN 2013ai & 2013-Apr-09 & B3000 & 1200 \\
\hline SN 2013ai & 2013-Apr-09 & R3000 & 1200 \\
\hline SN 2013ai & 2013-Mar-08 & B3000 & 1200 \\
\hline SN 2013ai & 2013-Mar-08 & R3000 & 1200 \\
\hline SN 2013ai & 2013-Mar-25 & B3000 & 1200 \\
\hline
\end{tabular}


Table A3. Continued.

\begin{tabular}{|c|c|c|c|}
\hline SN & $\begin{array}{c}\text { Observation } \\
\text { date }\end{array}$ & Grating & $\begin{array}{c}\text { Exposure } \\
\text { time (s) }\end{array}$ \\
\hline SN 2013ai & 2013-Mar-25 & R3000 & 1200 \\
\hline SN 2013ai & 2013-Мay-26 & В3000 & 1200 \\
\hline SN 2013ai & 2013-Мay-26 & R3000 & 1200 \\
\hline SN 2013aj & 2013-Mar-08 & В3000 & 1200 \\
\hline SN 2013aj & 2013-Mar-08 & R3000 & 1200 \\
\hline SN 2013aj & 2013-Mar-25 & B3000 & 1200 \\
\hline SN 2013aj & 2013-Mar-25 & R3000 & 1200 \\
\hline SN 2013am & 2013-Apr-09 & В3000 & 1200 \\
\hline SN 2013am & 2013-Apr-09 & R3000 & 1200 \\
\hline SN 2013am & 2013-Mar-24 & B3000 & 1200 \\
\hline SN 2013am & 2013-Mar-24 & В3000 & 1800 \\
\hline SN 2013am & 2013-Mar-24 & R3000 & 1200 \\
\hline SN 2013am & 2013-Mar-24 & R3000 & 1800 \\
\hline SN 2013am & 2013-Mar-25 & B3000 & 1200 \\
\hline SN 2013am & 2013-Mar-25 & R3000 & 1200 \\
\hline SN 2013am & 2013-Mar-27 & B3000 & 1500 \\
\hline SN 2013am & 2013-Mar-27 & R3000 & 1500 \\
\hline SN 2013ao & 2013-Mar-25 & В3000 & 1200 \\
\hline SN 2013ao & 2013-Mar-25 & R3000 & 1200 \\
\hline SN 2013bj & 2013-Apr-12 & B3000 & 1200 \\
\hline SN 2013bj & 2013-Apr-12 & R3000 & 1200 \\
\hline SN 2013bk & 2013-Apr-12 & В3000 & 1200 \\
\hline SN 2013bk & 2013-Apr-12 & R3000 & 1200 \\
\hline SN 2013by & 2013-Aug-19 & B3000 & 1200 \\
\hline SN 2013by & 2013-Aug-19 & R3000 & 1200 \\
\hline SN 2013by & 2013-Мay-26 & В3000 & 1200 \\
\hline SN 2013by & 2013-Мay-26 & R3000 & 1200 \\
\hline SN 2013by & 2014-Jul-08 & В3000 & 1200 \\
\hline SN 2013by & 2014-Jul-08 & R3000 & 1200 \\
\hline SN 2013bz & 2013-Мay-26 & B3000 & 1200 \\
\hline SN 2013bz & 2013-Мay-26 & R3000 & 1200 \\
\hline SN 2013cg & 2013-Мay-26 & В3000 & 1200 \\
\hline SN 2013cg & 2013-May-26 & R3000 & 1200 \\
\hline SN 2013cg & 2013-Мay-26 & R7000 & 1200 \\
\hline SN 2013cs & 2013-Мay-26 & В3000 & 1200 \\
\hline SN 2013cs & 2013-Мay-26 & R3000 & 1200 \\
\hline SN 2013cs & 2013-Мay-26 & R7000 & 1200 \\
\hline SN 2013cs & 2014-Mar-22 & В3000 & 1200 \\
\hline SN 2013cs & 2014-Mar-22 & R3000 & 1200 \\
\hline SN 2013cy & 2013-Jun-03 & В3000 & 1200 \\
\hline SN 2013cy & 2013-Jun-03 & R3000 & 1200 \\
\hline SN $2013 \mathrm{cz}$ & 2013-Jun-03 & B3000 & 1200 \\
\hline SN $2013 \mathrm{cz}$ & 2013-Jun-03 & R3000 & 1200 \\
\hline SN 2013df & 2013-Jun-22 & В3000 & 1200 \\
\hline SN 2013df & 2013-Jun-22 & R3000 & 1200 \\
\hline SN 2013dh & 2013-Jul-01 & В3000 & 1800 \\
\hline SN 2013dh & 2013-Jul-01 & R3000 & 1800 \\
\hline SN 2013dh & 2013-Jun-22 & В3000 & 1200 \\
\hline SN 2013dh & 2013-Jun-22 & R3000 & 1200 \\
\hline SN 2013du & 2013-Jul-04 & В3000 & 1200 \\
\hline SN 2013du & 2013-Jul-04 & R3000 & 1200 \\
\hline SN 2013ef & 2013-Jul-04 & В3000 & 1200 \\
\hline SN 2013ef & 2013-Jul-04 & R3000 & 1200 \\
\hline SN 2013ej & 2013-Aug-11 & В3000 & 1200 \\
\hline SN 2013ej & 2013-Aug-11 & R3000 & 1200 \\
\hline SN 2013ej & 2013-Aug-19 & B3000 & 1200 \\
\hline SN 2013ej & 2013-Aug-19 & R3000 & 1200 \\
\hline SN 2013ej & 2013-Jul-31 & B3000 & 1200 \\
\hline SN 2013ej & 2013-Jul-31 & R3000 & 1200 \\
\hline SN 2013ej & 2013-Oct-16 & В3000 & 1200 \\
\hline SN 2013ej & 2013-Oct-16 & R3000 & 1200 \\
\hline
\end{tabular}

Table A3. Continued.

\begin{tabular}{|c|c|c|c|}
\hline SN & $\begin{array}{c}\text { Observation } \\
\text { date }\end{array}$ & Grating & $\begin{array}{c}\text { Exposure } \\
\text { time (s) }\end{array}$ \\
\hline SN 2013ej & 2013-Oct-24 & B3000 & 1200 \\
\hline SN 2013ej & 2013-Oct-24 & R3000 & 1200 \\
\hline SN 2013ej & 2013-Sep-19 & B3000 & 1200 \\
\hline SN 2013ej & 2013-Sep-19 & R3000 & 1200 \\
\hline SN 2013ek & 2013-Aug-11 & B3000 & 1200 \\
\hline SN 2013ek & 2013-Aug-11 & R3000 & 1200 \\
\hline SN 2013ek & 2013-Aug-19 & B3000 & 1200 \\
\hline SN 2013ek & 2013-Aug-19 & R3000 & 1200 \\
\hline SN 2013ek & 2013-Jul-31 & B3000 & 1200 \\
\hline SN 2013ek & 2013-Jul-31 & R3000 & 1200 \\
\hline SN 2013ek & 2013-Sep-13 & B3000 & 1200 \\
\hline SN 2013ek & 2013-Sep-13 & R3000 & 1200 \\
\hline SN 2013er & 2013-Jul-31 & B3000 & 1200 \\
\hline SN 2013er & 2013-Jul-31 & R3000 & 1200 \\
\hline SN 2013fd & 2013-Sep-19 & B3000 & 1200 \\
\hline SN 2013fd & 2013-Sep-19 & R3000 & 1200 \\
\hline SN 2013fj & 2013-Sep-19 & B3000 & 1200 \\
\hline SN 2013fj & 2013-Sep-19 & R3000 & 1200 \\
\hline SN 2013fq & 2013-Oct-24 & B3000 & 1200 \\
\hline SN 2013fq & 2013-Oct-24 & R3000 & 1200 \\
\hline SN 2013fq & 2013-Sep-19 & B3000 & 1200 \\
\hline SN 2013fq & 2013-Sep-19 & R3000 & 1200 \\
\hline SN 2013fs & 2013-Nov-14 & B3000 & 1200 \\
\hline SN 2013fs & 2013-Nov-14 & R3000 & 1200 \\
\hline SN 2013fs & 2013-Nov-28 & B3000 & 1200 \\
\hline SN 2013fs & 2013-Nov-28 & R3000 & 1200 \\
\hline SN 2013fs & 2013-Oct-08 & B3000 & 1200 \\
\hline SN 2013fs & 2013-Oct-08 & R3000 & 1200 \\
\hline SN 2013fs & 2013-Oct-24 & B3000 & 1200 \\
\hline SN 2013fs & 2013-Oct-24 & R3000 & 1200 \\
\hline SN 2013ft & 2013-Oct-08 & B3000 & 1200 \\
\hline SN 2013ft & 2013-Oct-08 & R3000 & 1200 \\
\hline SN 2013fw & 2013-Nov-14 & B3000 & 1200 \\
\hline SN 2013fw & 2013-Nov-14 & R3000 & 1200 \\
\hline SN 2013gh & 2013-Aug-19 & B3000 & 1200 \\
\hline SN 2013gh & 2013-Aug-19 & R7000 & 1200 \\
\hline SN 2013gh & 2013-Aug-30 & B3000 & 1200 \\
\hline SN 2013gh & 2013-Aug-30 & R3000 & 1200 \\
\hline SN 2013gh & 2013-Aug-30 & R7000 & 1200 \\
\hline SN 2013gi & 2013-Nov-14 & B3000 & 1200 \\
\hline SN 2013gi & 2013-Nov-14 & R3000 & 1200 \\
\hline SN 2013gq & 2013-Oct-08 & B3000 & 1200 \\
\hline SN 2013gq & 2013-Oct-08 & R3000 & 1200 \\
\hline SN 2013gr & 2013-Dec-06 & B3000 & 1200 \\
\hline SN 2013gr & 2013-Dec-06 & R3000 & 1200 \\
\hline SN 2013gr & 2013-Dec-15 & B3000 & 1200 \\
\hline SN 2013gr & 2013-Dec-15 & R3000 & 1200 \\
\hline SN 2013gr & 2013-Dec-19 & B3000 & 1200 \\
\hline SN 2013gr & 2013-Dec-19 & R3000 & 1200 \\
\hline SN 2013gr & 2013-Dec-29 & B3000 & 1200 \\
\hline SN 2013gr & 2013-Dec-29 & R3000 & 1200 \\
\hline SN 2013gr & 2014-Jan-18 & B3000 & 1200 \\
\hline SN 2013gr & 2014-Jan-18 & R3000 & 1200 \\
\hline SN 2013hh & 2013-Dec-12 & B3000 & 1200 \\
\hline SN 2013hh & 2013-Dec-12 & R3000 & 1200 \\
\hline SN 2013hj & 2013-Dec-19 & B3000 & 1200 \\
\hline SN 2013hj & 2013-Dec-19 & R3000 & 1200 \\
\hline SN 2013hj & 2013-Dec-29 & B3000 & 1200 \\
\hline SN 2013hj & 2013-Dec-29 & R3000 & 1200 \\
\hline SN 2013hn & 2013-Dec-19 & B3000 & 1200 \\
\hline SN 2013hn & 2013-Dec-19 & R3000 & 1200 \\
\hline
\end{tabular}


Table A3. Continued.

\begin{tabular}{|c|c|c|c|}
\hline SN & $\begin{array}{c}\text { Observation } \\
\text { date }\end{array}$ & Grating & $\begin{array}{c}\text { Exposure } \\
\text { time (s) }\end{array}$ \\
\hline SN 2013hn & 2013-Dec-29 & B3000 & 1200 \\
\hline SN 2013hn & 2013-Dec-29 & R3000 & 1200 \\
\hline SN 2013hn & 2013-Jun-22 & B3000 & 1200 \\
\hline SN 2013hn & 2013-Jun-22 & R3000 & 1200 \\
\hline SN 2013hu & 2013-Dec-29 & B3000 & 1200 \\
\hline SN 2013hu & 2013-Dec-29 & R3000 & 1200 \\
\hline SN 2013hx & 2014-Feb-24 & B3000 & 1200 \\
\hline SN 2013hx & 2014-Feb-24 & R3000 & 1200 \\
\hline SN 2013hx & 2014-Jan-31 & B3000 & 1200 \\
\hline SN 2013hx & 2014-Jan-31 & R3000 & 1200 \\
\hline SN 2013hx & 2014-Mar-04 & B3000 & 1200 \\
\hline SN 2013hx & 2014-Mar-04 & R3000 & 1200 \\
\hline SN 2013hx & 2014-Mar-14 & B3000 & 1200 \\
\hline SN $2013 \mathrm{hx}$ & 2014-Mar-14 & R3000 & 1200 \\
\hline SN 2014D & 2014-Jan-18 & B3000 & 1200 \\
\hline SN 2014D & 2014-Jan-18 & R3000 & 1200 \\
\hline SN 2014E & 2014-Jan-18 & B3000 & 1200 \\
\hline SN 2014E & 2014-Jan-18 & R3000 & 1200 \\
\hline SN 2014I & 2014-Jan-18 & B3000 & 1200 \\
\hline SN 2014I & 2014-Jan-18 & R3000 & 1200 \\
\hline SN 2014L & 2014-Feb-24 & B3000 & 1200 \\
\hline SN 2014L & 2014-Feb-24 & R3000 & 1200 \\
\hline SN 2014L & 2014-Jan-31 & B3000 & 1200 \\
\hline SN 2014L & 2014-Jan-31 & R3000 & 1200 \\
\hline SN 2014L & 2014-Mar-14 & B3000 & 1200 \\
\hline SN 2014L & 2014-Mar-14 & R3000 & 1200 \\
\hline SN 2014L & 2014-Mar-22 & B3000 & 1200 \\
\hline SN 2014L & 2014-Mar-22 & R3000 & 1200 \\
\hline SN 2014N & 2014-Jan-31 & B3000 & 1200 \\
\hline SN 2014N & 2014-Jan-31 & R3000 & 1200 \\
\hline SN 2014X & 2014-Mar-04 & B3000 & 1200 \\
\hline SN 2014X & 2014-Mar-04 & R3000 & 1200 \\
\hline SN 2014aa & 2014-Apr-02 & B3000 & 1200 \\
\hline SN 2014aa & 2014-Apr-02 & R3000 & 1200 \\
\hline SN 2014aa & 2014-Mar-14 & B3000 & 1200 \\
\hline SN 2014aa & 2014-Mar-14 & R3000 & 1200 \\
\hline SN 2014aa & 2014-Mar-22 & B3000 & 1200 \\
\hline SN 2014aa & 2014-Mar-22 & R3000 & 1200 \\
\hline SN 2014ac & 2014-Mar-14 & В3000 & 1200 \\
\hline SN 2014ac & 2014-Mar-14 & R3000 & 1200 \\
\hline SN 2014ad & 2014-Apr-02 & В3000 & 1200 \\
\hline SN 2014ad & 2014-Apr-02 & R3000 & 1200 \\
\hline SN 2014ad & 2014-Apr-16 & В3000 & 1800 \\
\hline SN 2014ad & 2014-Apr-16 & R3000 & 1800 \\
\hline SN 2014ad & 2014-Apr-23 & В3000 & 1200 \\
\hline SN 2014ad & 2014-Apr-23 & R3000 & 1200 \\
\hline SN 2014ad & 2014-Mar-14 & В3000 & 1200 \\
\hline SN 2014ad & 2014-Mar-14 & R3000 & 1200 \\
\hline SN 2014ad & 2014-Mar-17 & В3000 & 1800 \\
\hline SN 2014ad & 2014-Mar-17 & R3000 & 1800 \\
\hline SN 2014ad & 2014-Mar-18 & B3000 & 1800 \\
\hline SN 2014ad & 2014-Mar-18 & R3000 & 1800 \\
\hline SN 2014ad & 2014-Mar-19 & B3000 & 1800 \\
\hline SN 2014ad & 2014-Mar-19 & R3000 & 1800 \\
\hline SN 2014ad & 2014-Mar-22 & В3000 & 1200 \\
\hline SN 2014ad & 2014-Mar-22 & R3000 & 1200 \\
\hline SN 2014ad & 2014-Mar-28 & В3000 & 1000 \\
\hline SN 2014ad & 2014-Mar-28 & R3000 & 1000 \\
\hline SN 2014ah & 2014-Apr-02 & B3000 & 1200 \\
\hline SN 2014ah & 2014-Apr-02 & R3000 & 1200 \\
\hline SN 2014ao & 2014-Apr-23 & В3000 & 1200 \\
\hline
\end{tabular}

Table A3. Continued.

\begin{tabular}{|c|c|c|c|}
\hline $\mathrm{SN}$ & $\begin{array}{l}\text { Observation } \\
\text { date }\end{array}$ & Grating & $\begin{array}{c}\text { Exposure } \\
\text { time (s) }\end{array}$ \\
\hline SN 2014ao & 2014-Apr-23 & R3000 & 1200 \\
\hline SN 2014ao & 2014-Apr-23 & R7000 & 1200 \\
\hline SN 2014ap & 2014-Mar-22 & B3000 & 1200 \\
\hline SN 2014ap & 2014-Mar-22 & R3000 & 1200 \\
\hline SN 2014at & 2014-Apr-23 & B3000 & 1200 \\
\hline SN 2014at & 2014-Apr-23 & R3000 & 1200 \\
\hline SN 2014at & 2014-Apr-23 & R7000 & 1200 \\
\hline SN 2014au & 2014-Apr-23 & B3000 & 1200 \\
\hline SN 2014au & 2014-Apr-23 & R3000 & 1200 \\
\hline SN 2014bx & 2014-Jul-22 & В3000 & 200 \\
\hline SN 2014bx & 2014-Jul-22 & R3000 & 200 \\
\hline SN 2014bz & 2014-Jun-11 & B3000 & 800 \\
\hline SN 2014bz & 2014-Jun-11 & R3000 & 800 \\
\hline SN 2014ca & 2014-Jun-11 & B3000 & 800 \\
\hline SN 2014ca & 2014-Jun-11 & R3000 & 800 \\
\hline SN $2014 \mathrm{~cm}$ & 2014-Jun-11 & B3000 & 800 \\
\hline SN $2014 \mathrm{~cm}$ & 2014-Jun-11 & R3000 & 800 \\
\hline SN 2014co & 2014-Jul-08 & B3000 & 1200 \\
\hline SN 2014co & 2014-Jul-08 & R3000 & 1200 \\
\hline SN 2014cp & 2014-Aug-05 & B3000 & 1200 \\
\hline SN 2014cp & 2014-Aug-05 & R3000 & 1200 \\
\hline SN 2014cp & 2014-Jul-08 & В3000 & 1200 \\
\hline SN 2014cp & 2014-Jul-08 & R3000 & 1200 \\
\hline SN 2014cq & 2014-Jul-08 & В3000 & 1200 \\
\hline SN 2014cq & 2014-Jul-08 & R3000 & 1200 \\
\hline SN 2014cr & 2014-Aug-05 & B3000 & 1200 \\
\hline SN 2014cr & 2014-Aug-05 & R3000 & 1200 \\
\hline SN 2014cr & 2014-Jul-08 & B3000 & 1200 \\
\hline SN 2014cr & 2014-Jul-08 & R3000 & 1200 \\
\hline SN 2014cs & 2014-Jul-08 & B3000 & 1200 \\
\hline SN 2014cs & 2014-Jul-08 & R3000 & 1200 \\
\hline SN 2014ct & 2014-Jun-12 & B3000 & 800 \\
\hline SN 2014ct & 2014-Jun-12 & R3000 & 800 \\
\hline SN 2014cu & 2014-Jul-08 & В3000 & 1200 \\
\hline SN 2014cu & 2014-Jul-08 & R3000 & 1200 \\
\hline SN 2014cw & 2014-Sep-09 & В3000 & 1200 \\
\hline SN 2014cw & 2014-Sep-09 & R3000 & 1200 \\
\hline SN 2014cx & 2014-Sep-09 & B3000 & 1200 \\
\hline SN 2014cx & 2014-Sep-09 & R3000 & 1200 \\
\hline SN 2014cx & 2014-Sep-10 & B3000 & 1200 \\
\hline SN 2014cx & 2014-Sep-10 & R3000 & 1200 \\
\hline SN 2014cy & 2014-Sep-09 & B3000 & 1200 \\
\hline SN 2014cy & 2014-Sep-09 & R3000 & 1200 \\
\hline SN 2014dc & 2014-Sep-10 & B3000 & 1200 \\
\hline SN 2014dc & 2014-Sep-10 & R3000 & 1200 \\
\hline SN 2014df & 2014-Jun-04 & B3000 & 900 \\
\hline SN 2014df & 2014-Jun-04 & R3000 & 900 \\
\hline SN 2014dl & 2014-Oct-03 & B3000 & 1200 \\
\hline SN 2014dl & 2014-Oct-03 & R3000 & 1200 \\
\hline SN 2014dn & 2014-Oct-03 & B3000 & 1200 \\
\hline SN 2014dn & 2014-Oct-03 & R3000 & 1200 \\
\hline SN 2014dn & 2014-Oct-09 & B3000 & 1200 \\
\hline SN 2014dn & 2014-Oct-09 & R3000 & 1200 \\
\hline SN 2014do & 2014-Oct-03 & B3000 & 1200 \\
\hline SN 2014do & 2014-Oct-03 & R3000 & 1200 \\
\hline SN 2014dp & 2014-Oct-09 & B3000 & 1200 \\
\hline SN 2014dp & 2014-Oct-09 & R3000 & 1200 \\
\hline SN 2014dq & 2014-Nov-14 & B3000 & 1200 \\
\hline SN 2014dq & 2014-Nov-14 & R3000 & 1200 \\
\hline SN 2014dq & 2014-Oct-27 & B3000 & 1200 \\
\hline SN 2014dq & 2014-Oct-27 & R3000 & 1200 \\
\hline
\end{tabular}


Table A3. Continued.

\begin{tabular}{|c|c|c|c|}
\hline SN & $\begin{array}{c}\text { Observation } \\
\text { date }\end{array}$ & Grating & $\begin{array}{c}\text { Exposure } \\
\text { time (s) }\end{array}$ \\
\hline SN 2014dr & 2014-Oct-18 & B3000 & 1200 \\
\hline SN 2014dr & 2014-Oct-18 & R3000 & 1200 \\
\hline SN 2014eg & 2015-Jan-29 & B3000 & 1200 \\
\hline SN 2014eg & 2015-Jan-29 & R7000 & 1200 \\
\hline SN 2014eg & 2015-Mar-05 & B3000 & 1200 \\
\hline SN 2014eg & 2015-Mar-05 & R3000 & 1200 \\
\hline SN 2015F & 2015-Apr-11 & B3000 & 1200 \\
\hline SN $2015 \mathrm{~F}$ & 2015-Apr-11 & R3000 & 1200 \\
\hline SN 2015F & 2015-Apr-29 & B3000 & 1200 \\
\hline SN 2015F & 2015-Apr-29 & R3000 & 1200 \\
\hline SN 2015F & 2015-Jul-08 & B3000 & 1200 \\
\hline SN 2015F & 2015-Jul-08 & R3000 & 1200 \\
\hline SN 2015F & 2015-Mar-12 & B3000 & 1200 \\
\hline SN 2015F & 2015-Mar-12 & R3000 & 1200 \\
\hline SN 2015J & 2015-Aug-17 & B3000 & 1200 \\
\hline SN 2015J & 2015-Aug-17 & R7000 & 1200 \\
\hline SN 2015J & 2015-Jul-01 & B3000 & 1200 \\
\hline SN 2015J & 2015-Jul-01 & R7000 & 1200 \\
\hline SN 2015J & 2015-Jul-08 & B3000 & 1200 \\
\hline SN 2015J & 2015-Jul-08 & R7000 & 1200 \\
\hline SN 2015J & 2015-Jul-25 & B3000 & 900 \\
\hline SN 2015J & 2015-Jul-25 & R3000 & 900 \\
\hline SN 2015J & 2015-Jul-31 & B3000 & 1200 \\
\hline SN 2015J & 2015-Jul-31 & R3000 & 1200 \\
\hline SN 2015J & 2015-Jun-24 & B3000 & 1200 \\
\hline SN 2015J & 2015-Jun-24 & R3000 & 1200 \\
\hline ASASSN-13co & 2013-Sep-19 & B3000 & 1200 \\
\hline ASASSN-13co & 2013-Sep-19 & R3000 & 1200 \\
\hline ASASSN-14dp & 2014-Jul-08 & B3000 & 1200 \\
\hline ASASSN-14dp & 2014-Jul-08 & R3000 & 1200 \\
\hline ASASSN-14eu & 2014-Aug-05 & B3000 & 1200 \\
\hline ASASSN-14eu & 2014-Aug-05 & R3000 & 1200 \\
\hline ASASSN-14ew & 2014-Sep-10 & B3000 & 1200 \\
\hline ASASSN-14ew & 2014-Sep-10 & R3000 & 1200 \\
\hline ASASSN-14fo & 2014-Sep-10 & B3000 & 1200 \\
\hline ASASSN-14fo & 2014-Sep-10 & R3000 & 1200 \\
\hline ASASSN-14fw & 2014-Sep-10 & B3000 & 1200 \\
\hline ASASSN-14fw & 2014-Sep-10 & R3000 & 1200 \\
\hline ASASSN-14gs & 2014-Sep-09 & B3000 & 1200 \\
\hline ASASSN-14gs & 2014-Sep-09 & R3000 & 1200 \\
\hline ASASSN-14ha & 2014-Nov-06 & B3000 & 1200 \\
\hline ASASSN-14ha & 2014-Nov-06 & R3000 & 1200 \\
\hline ASASSN-14ha & 2014-Oct-03 & B3000 & 1200 \\
\hline ASASSN-14ha & 2014-Oct-03 & R3000 & 1200 \\
\hline ASASSN-14ha & 2014-Oct-09 & B3000 & 1200 \\
\hline ASASSN-14ha & 2014-Oct-09 & R3000 & 1200 \\
\hline ASASSN-14ha & 2014-Oct-18 & B3000 & 1200 \\
\hline ASASSN-14ha & 2014-Oct-18 & R3000 & 1200 \\
\hline ASASSN-14ha & 2014-Oct-27 & B3000 & 1200 \\
\hline ASASSN-14ha & 2014-Oct-27 & R3000 & 1200 \\
\hline ASASSN-14hr & 2014-Oct-03 & B3000 & 1200 \\
\hline ASASSN-14hr & 2014-Oct-03 & R3000 & 1200 \\
\hline ASASSN-14hu & 2014-Nov-06 & B3000 & 1200 \\
\hline ASASSN-14hu & 2014-Nov-06 & R3000 & 1200 \\
\hline ASASSN-14hu & 2014-Oct-03 & B3000 & 1200 \\
\hline ASASSN-14hu & 2014-Oct-03 & R3000 & 1200 \\
\hline ASASSN-14hu & 2014-Oct-09 & B3000 & 1200 \\
\hline ASASSN-14hu & 2014-Oct-09 & R3000 & 1200 \\
\hline ASASSN-14hu & 2014-Oct-18 & B3000 & 1200 \\
\hline ASASSN-14hu & 2014-Oct-18 & R3000 & 1200 \\
\hline ASASSN-14hu & 2014-Oct-27 & B3000 & 1200 \\
\hline
\end{tabular}

Table A3. Continued.

\begin{tabular}{|c|c|c|c|}
\hline SN & $\begin{array}{c}\text { Observation } \\
\text { date }\end{array}$ & Grating & $\begin{array}{c}\text { Exposure } \\
\text { time (s) }\end{array}$ \\
\hline ASASSN-14hu & 2014-Oct-27 & R3000 & 1200 \\
\hline ASASSN-14ig & 2014-Oct-03 & B3000 & 1200 \\
\hline ASASSN-14ig & 2014-Oct-03 & R3000 & 1200 \\
\hline ASASSN-14ih & 2014-Oct-03 & B3000 & 1200 \\
\hline ASASSN-14ih & 2014-Oct-03 & R3000 & 1200 \\
\hline ASASSN-14ii & 2014-Oct-03 & B3000 & 1200 \\
\hline ASASSN-14ii & 2014-Oct-03 & R3000 & 1200 \\
\hline ASASSN-14il & 2014-Oct-03 & B3000 & 1200 \\
\hline ASASSN-14il & 2014-Oct-03 & R3000 & 1200 \\
\hline ASASSN-14il & 2014-Oct-09 & B3000 & 1200 \\
\hline ASASSN-14il & 2014-Oct-09 & R3000 & 1200 \\
\hline ASASSN-14il & 2014-Oct-18 & B3000 & 1200 \\
\hline ASASSN-14il & 2014-Oct-18 & R3000 & 1200 \\
\hline ASASSN-14il & 2014-Oct-27 & B3000 & 1200 \\
\hline ASASSN-14il & 2014-Oct-27 & R3000 & 1200 \\
\hline ASASSN-14jc & 2014-Nov-06 & B3000 & 1200 \\
\hline ASASSN-14jc & 2014-Nov-06 & R3000 & 1200 \\
\hline ASASSN-14jc & 2014-Oct-27 & B3000 & 1200 \\
\hline ASASSN-14jc & 2014-Oct-27 & R3000 & 1200 \\
\hline ASASSN-14jg & 2014-Nov-06 & B3000 & 1200 \\
\hline ASASSN-14jg & 2014-Nov-06 & R3000 & 1200 \\
\hline ASASSN-14ko & 2014-Dec-16 & B3000 & 1200 \\
\hline ASASSN-14ko & 2014-Dec-16 & R7000 & 1200 \\
\hline ASASSN-14ko & 2015-Feb-15 & B3000 & 1200 \\
\hline ASASSN-14ko & 2015-Feb-15 & R3000 & 1200 \\
\hline ASASSN-14ko & 2015-Jan-15 & B3000 & 1200 \\
\hline ASASSN-14ko & 2015-Jan-15 & R7000 & 1200 \\
\hline ASASSN-14kp & 2014-Nov-26 & B3000 & 1200 \\
\hline ASASSN-14kp & 2014-Nov-26 & R3000 & 1200 \\
\hline ASASSN-14kq & 2014-Nov-26 & B3000 & 1200 \\
\hline ASASSN-14kq & 2014-Nov-26 & R3000 & 1200 \\
\hline ASASSN-14lp & 2015-Apr-29 & B3000 & 1200 \\
\hline ASASSN-14lp & 2015-Apr-29 & R3000 & 1200 \\
\hline ASASSN-14lp & 2015-Jul-01 & B3000 & 1200 \\
\hline ASASSN-14lp & 2015-Jul-01 & R3000 & 1200 \\
\hline ASASSN-14lt & 2014-Dec-16 & B3000 & 1200 \\
\hline ASASSN-14lt & 2014-Dec-16 & R3000 & 1200 \\
\hline ASASSN-14lw & 2014-Dec-16 & B3000 & 1200 \\
\hline ASASSN-14lw & 2014-Dec-16 & R3000 & 1200 \\
\hline ASASSN-14lw & 2015-Feb-15 & B3000 & 1200 \\
\hline ASASSN-14lw & 2015-Feb-15 & R3000 & 1200 \\
\hline ASASSN-14lw & 2015-Jan-06 & B3000 & 1200 \\
\hline ASASSN-14lw & 2015-Jan-06 & R3000 & 1200 \\
\hline ASASSN-14lw & 2015-Jun-24 & B3000 & 1200 \\
\hline ASASSN-14lw & 2015-Jun-24 & R3000 & 1200 \\
\hline ASASSN-15aj & 2015-Feb-15 & B3000 & 1200 \\
\hline ASASSN-15aj & 2015-Feb-15 & R3000 & 1200 \\
\hline ASASSN-15aj & 2015-Feb-24 & B3000 & 1200 \\
\hline ASASSN-15aj & 2015-Feb-24 & R3000 & 1200 \\
\hline ASASSN-15aj & 2015-Mar-05 & B3000 & 1200 \\
\hline ASASSN-15aj & 2015-Mar-05 & R3000 & 1200 \\
\hline ASASSN-15al & 2015-Jan-15 & B3000 & 1200 \\
\hline ASASSN-15al & 2015-Jan-15 & R7000 & 1200 \\
\hline ASASSN-15as & 2015-Jan-16 & B3000 & 1200 \\
\hline ASASSN-15as & 2015-Jan-16 & R7000 & 1200 \\
\hline ASASSN-15az & 2015-Jan-16 & B3000 & 1200 \\
\hline ASASSN-15az & 2015-Jan-16 & R7000 & 1200 \\
\hline ASASSN-15ba & 2015-Feb-15 & B3000 & 1200 \\
\hline ASASSN-15ba & 2015-Feb-15 & R3000 & 1200 \\
\hline ASASSN-15ba & 2015-Feb-24 & B3000 & 1200 \\
\hline ASASSN-15ba & 2015-Feb-24 & R3000 & 1200 \\
\hline
\end{tabular}


Table A3. Continued.

\begin{tabular}{|c|c|c|c|}
\hline SN & $\begin{array}{c}\text { Observation } \\
\text { date }\end{array}$ & Grating & $\begin{array}{c}\text { Exposure } \\
\text { time (s) }\end{array}$ \\
\hline ASASSN-15ba & 2015-Jan-25 & B3000 & 1200 \\
\hline ASASSN-15ba & 2015-Jan-25 & R3000 & 1200 \\
\hline ASASSN-15ba & 2015-Jan-29 & B3000 & 1200 \\
\hline ASASSN-15ba & 2015-Jan-29 & R3000 & 1200 \\
\hline ASASSN-15ba & 2015-Jul-08 & B3000 & 1200 \\
\hline ASASSN-15ba & 2015-Jul-08 & R3000 & 1200 \\
\hline ASASSN-15ba & 2015-Mar-12 & B3000 & 1200 \\
\hline ASASSN-15ba & 2015-Mar-12 & R3000 & 1200 \\
\hline ASASSN-15dz & 2015-Mar-05 & B3000 & 1200 \\
\hline ASASSN-15dz & 2015-Mar-05 & R3000 & 1200 \\
\hline ASASSN-15eb & 2015-Mar-05 & B3000 & 1200 \\
\hline ASASSN-15eb & 2015-Mar-05 & R3000 & 1200 \\
\hline ASASSN-15ek & 2015-Mar-12 & B3000 & 1200 \\
\hline ASASSN-15ek & 2015-Mar-12 & R3000 & 1200 \\
\hline ASASSN-15go & 2015-Apr-11 & B3000 & 1200 \\
\hline ASASSN-15go & 2015-Apr-11 & R3000 & 1200 \\
\hline ASASSN-15hs & 2015-Apr-29 & B3000 & 1200 \\
\hline ASASSN-15hs & 2015-Apr-29 & R3000 & 1200 \\
\hline ASASSN-15hx & 2015-Apr-29 & B3000 & 1200 \\
\hline ASASSN-15hx & 2015-Apr-29 & R3000 & 1200 \\
\hline ASASSN-15hx & 2015-Aug-17 & В3000 & 600 \\
\hline ASASSN-15hx & 2015-Aug-17 & R3000 & 600 \\
\hline ASASSN-15hx & 2015-Jul-08 & B3000 & 1200 \\
\hline ASASSN-15hx & 2015-Jul-08 & R3000 & 1200 \\
\hline ASASSN-15hx & 2015-Jul-31 & B3000 & 1200 \\
\hline ASASSN-15hx & 2015-Jul-31 & R3000 & 1200 \\
\hline ASASSN-15hx & 2015-Jun-24 & B3000 & 1200 \\
\hline ASASSN-15hx & 2015-Jun-24 & R3000 & 1200 \\
\hline ASASSN-15hy & 2015-Apr-29 & B3000 & 1200 \\
\hline ASASSN-15hy & 2015-Apr-29 & R3000 & 1200 \\
\hline ASASSN-15hy & 2015-Aug-17 & В3000 & 1200 \\
\hline ASASSN-15hy & 2015-Aug-17 & R3000 & 1200 \\
\hline ASASSN-15hy & 2015-Jul-01 & B3000 & 1200 \\
\hline ASASSN-15hy & 2015-Jul-01 & R3000 & 1200 \\
\hline ASASSN-15hy & 2015-Jul-08 & B3000 & 1200 \\
\hline ASASSN-15hy & 2015-Jul-08 & R3000 & 1200 \\
\hline ASASSN-15hy & 2015-Jul-31 & B3000 & 1200 \\
\hline ASASSN-15hy & 2015-Jul-31 & R3000 & 1200 \\
\hline ASASSN-15hy & 2015-Jun-24 & В3000 & 1200 \\
\hline ASASSN-15hy & 2015-Jun-24 & R3000 & 1200 \\
\hline ASASSN-15hy & 2015-Мay-03 & B3000 & 900 \\
\hline ASASSN-15hy & 2015-Мay-03 & R3000 & 900 \\
\hline ASASSN-15hy & 2015-Мay-07 & B3000 & 1200 \\
\hline ASASSN-15hy & 2015-Мay-07 & R3000 & 1200 \\
\hline ASASSN-15hy & 2015-May-13 & В3000 & 600 \\
\hline ASASSN-15hy & 2015-May-13 & R3000 & 600 \\
\hline ASASSN-15hz & 2015-Apr-29 & B3000 & 1200 \\
\hline ASASSN-15hz & 2015-Apr-29 & R3000 & 1200 \\
\hline ASASSN-15ic & 2015-Apr-29 & B3000 & 1200 \\
\hline ASASSN-15ic & 2015-Apr-29 & R3000 & 1200 \\
\hline ASASSN-15lh & 2015-Jul-01 & B3000 & 1200 \\
\hline ASASSN-15lh & 2015-Jul-01 & R3000 & 1200 \\
\hline ASASSN-15lh & 2015-Jul-08 & B3000 & 1200 \\
\hline ASASSN-15lh & 2015-Jul-08 & R3000 & 1200 \\
\hline ASASSN-15lh & 2015-Jul-31 & B3000 & 1200 \\
\hline ASASSN-15lh & 2015-Jul-31 & R3000 & 1200 \\
\hline ASASSN-15lh & 2015-Jun-24 & B3000 & 1200 \\
\hline ASASSN-15lh & 2015-Jun-24 & R3000 & 1200 \\
\hline ASASSN-15lv & 2015-Jul-01 & B3000 & 1200 \\
\hline ASASSN-15lv & 2015-Jul-01 & R3000 & 1200 \\
\hline CSS1222 & 2014-Apr-02 & B3000 & 1200 \\
\hline
\end{tabular}

Table A3. Continued.

\begin{tabular}{|c|c|c|c|}
\hline SN & $\begin{array}{c}\text { Observation } \\
\text { date }\end{array}$ & Grating & $\begin{array}{c}\text { Exposure } \\
\text { time (s) }\end{array}$ \\
\hline CSS1222 & 2014-Apr-02 & R3000 & 1200 \\
\hline Gaia15agm & 2015-Jul-01 & B3000 & 1200 \\
\hline Gaia15agm & 2015-Jul-01 & R3000 & 1200 \\
\hline LSQ12dlf & 2012-Aug-11 & В3000 & 1200 \\
\hline LSQ12dlf & 2012-Aug-11 & R3000 & 1200 \\
\hline LSQ12dlf & 2012-Aug-12 & B3000 & 1200 \\
\hline LSQ12dlf & 2012-Aug-12 & R3000 & 1200 \\
\hline LSQ12dlf & 2012-Aug-13 & B3000 & 1200 \\
\hline LSQ12dlf & 2012-Aug-13 & R3000 & 1200 \\
\hline LSQ12ege & 2012-Aug-11 & В3000 & 1200 \\
\hline LSQ12ege & 2012-Aug-11 & R3000 & 1200 \\
\hline LSQ12ege & 2012-Aug-12 & B3000 & 1200 \\
\hline LSQ12ege & 2012-Aug-12 & R3000 & 1200 \\
\hline LSQ12ege & 2012-Aug-13 & B3000 & 1200 \\
\hline LSQ12ege & 2012-Aug-13 & R3000 & 1200 \\
\hline LSQ12ehh & 2012-Aug-11 & B3000 & 1200 \\
\hline LSQ12ehh & 2012-Aug-11 & R3000 & 1200 \\
\hline LSQ12ehh & 2012-Aug-12 & B3000 & 1200 \\
\hline LSQ12ehh & 2012-Aug-12 & R3000 & 1200 \\
\hline LSQ12ehh & 2012-Aug-13 & B3000 & 1200 \\
\hline LSQ12ehh & 2012-Aug-13 & R3000 & 1200 \\
\hline LSQ12ers & 2012-Sep-06 & В3000 & 1200 \\
\hline LSQ12ers & 2012-Sep-06 & R3000 & 1200 \\
\hline LSQ12euu & 2012-Sep-06 & B3000 & 1200 \\
\hline LSQ12euu & 2012-Sep-06 & R3000 & 1200 \\
\hline LSQ12eve & 2012-Sep-06 & B3000 & 1200 \\
\hline LSQ12eve & 2012-Sep-06 & R3000 & 1200 \\
\hline LSQ12gdj & 2012-Nov-19 & B3000 & 1200 \\
\hline LSQ12gdj & 2012-Nov-19 & R3000 & 1200 \\
\hline LSQ12gdj & 2012-Nov-20 & B3000 & 1200 \\
\hline LSQ12gdj & 2012-Nov-20 & R3000 & 1200 \\
\hline LSQ12gdj & 2012-Nov-21 & B3000 & 1200 \\
\hline LSQ12gdj & 2012-Nov-21 & R3000 & 1200 \\
\hline LSQ13anm & 2013-May-26 & B3000 & 1200 \\
\hline LSQ13anm & 2013-May-26 & R3000 & 1200 \\
\hline LSQ13ddu & 2013-Dec-06 & В3000 & 1200 \\
\hline LSQ13ddu & 2013-Dec-06 & R3000 & 1200 \\
\hline LSQ13ddu & 2013-Dec-15 & В3000 & 1200 \\
\hline LSQ13ddu & 2013-Dec-15 & R3000 & 1200 \\
\hline LSQ13ddu & 2013-Dec-19 & B3000 & 1200 \\
\hline LSQ13ddu & 2013-Dec-19 & R3000 & 1200 \\
\hline LSQ13pp & 2013-Mar-25 & B3000 & 1200 \\
\hline LSQ13pp & 2013-Mar-25 & R3000 & 1200 \\
\hline LSQ14bcj & 2014-Apr-23 & B3000 & 1200 \\
\hline LSQ14bcj & 2014-Apr-23 & R3000 & 1200 \\
\hline LSQ14bcj & 2014-Apr-23 & $\mathrm{R} 7000$ & 1200 \\
\hline LSQ15adm & 2015-Apr-29 & B3000 & 1200 \\
\hline LSQ15adm & 2015-Apr-29 & R3000 & 1200 \\
\hline LSQ15adm & 2015-Aug-17 & B3000 & 1200 \\
\hline LSQ15adm & 2015-Aug-17 & R3000 & 1200 \\
\hline LSQ15adm & 2015-Jul-01 & В3000 & 1200 \\
\hline LSQ15adm & 2015-Jul-01 & R3000 & 1200 \\
\hline LSQ15adm & 2015-Jul-08 & B3000 & 1200 \\
\hline LSQ15adm & 2015-Jul-08 & R3000 & 1200 \\
\hline LSQ15adm & 2015-Jul-31 & B3000 & 1200 \\
\hline LSQ15adm & 2015-Jul-31 & R3000 & 1200 \\
\hline LSQ15adm & 2015-Jun-24 & B3000 & 1060 \\
\hline LSQ15adm & 2015-Jun-24 & R3000 & 1060 \\
\hline LSQ15adm & 2015-Мay-07 & B3000 & 1330 \\
\hline LSQ15adm & 2015-May-07 & R3000 & 1330 \\
\hline LSQ15adm & 2015-Мay-14 & B3000 & 1800 \\
\hline
\end{tabular}


Table A3. Continued.

\begin{tabular}{|c|c|c|c|}
\hline SN & $\begin{array}{c}\text { Observation } \\
\text { date }\end{array}$ & Grating & $\begin{array}{c}\text { Exposure } \\
\text { time (s) }\end{array}$ \\
\hline LSQ15adm & 2015-May-14 & R3000 & 1800 \\
\hline LSQ15ey & 2015-Jan-29 & B3000 & 1200 \\
\hline LSQ15ey & 2015-Jan-29 & R3000 & 1200 \\
\hline MASTERJ1408 & 2014-Apr-23 & B3000 & 1200 \\
\hline MASTERJ1408 & 2014-Apr-23 & R7000 & 1200 \\
\hline MASTERJ1353 & 2015-Mar-05 & B3000 & 1200 \\
\hline MASTERJ1353 & 2015-Mar-05 & R3000 & 1200 \\
\hline MASTERJ0746 & 2015-Apr-29 & B3000 & 1200 \\
\hline MASTERJ0746 & 2015-Apr-29 & R3000 & 1200 \\
\hline OGLE-2013-SN-070 & 2013-Oct-08 & B3000 & 1200 \\
\hline OGLE-2013-SN-070 & 2013-Oct-08 & R3000 & 1200 \\
\hline OGLE-2014-SN-019 & 2014-Feb-24 & B3000 & 1200 \\
\hline OGLE-2014-SN-019 & 2014-Feb-24 & R3000 & 1200 \\
\hline OGLE-2014-SN-019 & 2014-Mar-14 & B3000 & 1200 \\
\hline OGLE-2014-SN-019 & 2014-Mar-14 & R3000 & 1200 \\
\hline OGLE-2014-SN-021 & 2014-Feb-24 & B3000 & 1200 \\
\hline OGLE-2014-SN-021 & 2014-Feb-24 & R3000 & 1200 \\
\hline OGLE-2014-SN-021 & 2014-Mar-14 & B3000 & 1200 \\
\hline OGLE-2014-SN-021 & 2014-Mar-14 & R3000 & 1200 \\
\hline OGLE-2014-SN-067 & 2014-Aug-29 & B3000 & 1200 \\
\hline OGLE-2014-SN-067 & 2014-Aug-29 & R3000 & 1200 \\
\hline PS1-14lz & 2014-Feb-24 & B3000 & 1200 \\
\hline PS1-14lz & 2014-Feb-24 & R3000 & 1200 \\
\hline PS1-14md & 2014-Feb-24 & B3000 & 1200 \\
\hline PS1-14md & 2014-Feb-24 & R3000 & 1200 \\
\hline PS1-14ra & 2014-Mar-14 & B3000 & 1200 \\
\hline PS1-14ra & 2014-Mar-14 & R3000 & 1200 \\
\hline PS1-14vw & 2014-Apr-02 & B3000 & 1200 \\
\hline PS1-14vw & 2014-Apr-02 & R3000 & 1200 \\
\hline PS1-14vx & 2014-Apr-02 & B3000 & 1200 \\
\hline PS1-14vx & 2014-Apr-02 & R3000 & 1200 \\
\hline PS1-14wl & 2014-Apr-02 & B3000 & 1200 \\
\hline PS1-14wl & 2014-Apr-02 & R3000 & 1200 \\
\hline PS15ae & 2015-Feb-24 & B3000 & 1200 \\
\hline PS15ae & 2015-Feb-24 & R3000 & 1200 \\
\hline PS15ae & 2015-Mar-12 & B3000 & 1200 \\
\hline PS15ae & 2015-Mar-12 & R3000 & 1200 \\
\hline PS15br & 2015-Mar-12 & B3000 & 1200 \\
\hline PS15br & 2015-Mar-12 & R3000 & 1200 \\
\hline PSNJ02454138-0812301 & 2014-Oct-27 & B3000 & 1200 \\
\hline PSNJ02454138-0812301 & 2014-Oct-27 & $\mathrm{R} 3000$ & 1200 \\
\hline PSNJ05031639-0256110 & 2014-Nov-06 & B3000 & 1200 \\
\hline PSNJ05031639-0256110 & 2014-Nov-06 & R3000 & 1200 \\
\hline PSNJ09023787+2556042 & 2015-Apr-11 & B3000 & 1200 \\
\hline PSNJ09023787+2556042 & 2015-Apr-11 & R3000 & 1200 \\
\hline PSNJ09204691-0803340 & 2014-Dec-16 & B3000 & 1200 \\
\hline PSNJ09204691-0803340 & 2014-Dec-16 & $\mathrm{R} 7000$ & 1200 \\
\hline PSNJ09204691-0803340 & 2014-Nov-26 & B3000 & 1200 \\
\hline PSNJ09204691-0803340 & 2014-Nov-26 & R3000 & 1200 \\
\hline PSNJ09204691-0803340 & 2015-Feb-15 & B3000 & 1200 \\
\hline PSNJ09204691-0803340 & 2015-Feb-15 & R3000 & 1200 \\
\hline PSNJ09204691-0803340 & 2015-Jan-15 & B3000 & 1200 \\
\hline PSNJ09204691-0803340 & 2015-Jan-15 & R7000 & 1200 \\
\hline PSNJ09204691-0803340 & 2015-Jan-16 & B3000 & 1200 \\
\hline PSNJ09204691-0803340 & 2015-Jan-16 & R7000 & 1200 \\
\hline PSNJ10433393-3048206 & 2015-Feb-15 & B3000 & 1200 \\
\hline PSNJ10433393-3048206 & 2015-Feb-15 & R3000 & 1200 \\
\hline PSNJ11484578-2817312 & 2013-Dec-06 & B3000 & 1200 \\
\hline PSNJ11484578-2817312 & 2013-Dec-06 & R3000 & 1200 \\
\hline PSNJ13471211-2422171 & 2015-Feb-15 & B3000 & 1200 \\
\hline PSNJ13471211-2422171 & 2015-Feb-15 & R3000 & 1200 \\
\hline
\end{tabular}

Table A3. Continued.

\begin{tabular}{|c|c|c|c|}
\hline $\mathrm{SN}$ & $\begin{array}{c}\text { Observation } \\
\text { date }\end{array}$ & Grating & $\begin{array}{c}\text { Exposure } \\
\text { time (s) }\end{array}$ \\
\hline PSNJ17194328-7721305 & 2013-Aug-30 & B3000 & 1200 \\
\hline PSNJ17194328-7721305 & 2013-Aug-30 & R3000 & 1200 \\
\hline PSNJ17194328-7721305 & 2013-Aug-30 & $\mathrm{R} 7000$ & 1200 \\
\hline PSNJ17194328-7721305 & 2014-Sep-09 & B3000 & 1200 \\
\hline PSNJ17194328-7721305 & 2014-Sep-09 & R3000 & 1200 \\
\hline PSNJ17285509-6609103 & 2013-Aug-30 & B3000 & 1200 \\
\hline PSNJ17285509-6609103 & 2013-Aug-30 & R3000 & 1200 \\
\hline PSNJ19251269-5336028 & 2015-Aug-17 & В3000 & 1200 \\
\hline PSNJ19251269-5336028 & 2015-Aug-17 & R3000 & 1200 \\
\hline PSNJ20250386-2449133 & 2014-Nov-06 & B3000 & 1200 \\
\hline PSNJ20250386-2449133 & 2014-Nov-06 & R3000 & 1200 \\
\hline PSNJ20250386-2449133 & 2014-Nov-14 & B3000 & 1200 \\
\hline PSNJ20250386-2449133 & 2014-Nov-14 & R3000 & 1200 \\
\hline PSNJ23320218-4545032 & 2012-Sep-07 & B3000 & 1200 \\
\hline PSNJ23320218-4545032 & 2012-Sep-07 & R3000 & 1200 \\
\hline PTF12gzk & 2012-Aug-10 & B3000 & 1200 \\
\hline PTF12gzk & 2012-Aug-10 & R3000 & 1200 \\
\hline PTF12gzk & 2012-Aug-11 & В3000 & 1200 \\
\hline PTF12gzk & 2012-Aug-11 & R3000 & 1200 \\
\hline PTF12gzk & 2012-Aug-12 & В3000 & 1200 \\
\hline PTF12gzk & 2012-Aug-12 & R3000 & 1200 \\
\hline PTF12gzk & 2012-Aug-13 & B3000 & 1200 \\
\hline PTF12gzk & 2012-Aug-13 & R3000 & 1200 \\
\hline PTF12gzk & 2012-Sep-06 & B3000 & 1200 \\
\hline PTF12gzk & 2012-Sep-06 & R3000 & 1200 \\
\hline PTF12gzk & 2012-Sep-07 & B3000 & 1200 \\
\hline PTF12gzk & 2012-Sep-07 & R3000 & 1200 \\
\hline PTF12gzk & 2012-Sep-23 & B3000 & 1200 \\
\hline PTF12gzk & 2012-Sep-23 & R3000 & 1200 \\
\hline iPTF13bvn & 2013-Jun-22 & B3000 & 1200 \\
\hline iPTF13bvn & 2013-Jun-22 & R3000 & 1200 \\
\hline iPTF13dge & 2013-Dec-06 & B3000 & 1200 \\
\hline iPTF13dge & 2013-Dec-06 & R3000 & 1200 \\
\hline iPTF13dge & 2013-Oct-08 & B3000 & 1200 \\
\hline iPTF13dge & 2013-Oct-08 & R3000 & 1200 \\
\hline iPTF13dge & 2013-Oct-16 & B3000 & 1200 \\
\hline iPTF13dge & 2013-Oct-16 & R3000 & 1200 \\
\hline iPTF13dge & 2013-Sep-13 & B3000 & 1200 \\
\hline iPTF13dge & 2013-Sep-13 & R3000 & 1200 \\
\hline iPTF13dge & 2013-Sep-19 & B3000 & 1200 \\
\hline iPTF13dge & 2013-Sep-19 & R3000 & 1200 \\
\hline iPTF13dge & 2013-Sep-19 & R7000 & 1200 \\
\hline SMTJ10310056-3658262 & 2015-Apr-11 & B3000 & 1200 \\
\hline SMTJ10310056-3658262 & 2015-Apr-11 & R3000 & 1200 \\
\hline SMTJ03253351-5344190 & 2013-Dec-06 & B3000 & 1200 \\
\hline SMTJ03253351-5344190 & 2013-Dec-06 & R3000 & 1200 \\
\hline SMTJ05451320-4735425 & 2014-Jan-13 & B3000 & 900 \\
\hline SMTJ05451320-4735425 & 2014-Jan-13 & R3000 & 900 \\
\hline SMTJ13494831-0658174 & 2015-Aug-17 & B3000 & 900 \\
\hline SMTJ13494831-0658174 & 2015-Aug-17 & R7000 & 900 \\
\hline SNhunt222 & 2013-Dec-19 & B3000 & 1200 \\
\hline SNhunt 222 & 2013-Dec-19 & R3000 & 1200 \\
\hline SNhunt239 & 2014-Apr-02 & В3000 & 1200 \\
\hline SNhunt239 & 2014-Apr-02 & R3000 & 1200 \\
\hline SNhunt259 & 2014-Oct-18 & B3000 & 1200 \\
\hline SNhunt259 & 2014-Oct-18 & R3000 & 1200 \\
\hline SNhunt261 & 2014-Oct-27 & B3000 & 1200 \\
\hline SNhunt261 & 2014-Oct-27 & R3000 & 1200 \\
\hline
\end{tabular}


Table A4. Fits of Na line in SNe Ia.

\begin{tabular}{lccccc}
\hline \hline SN & Observation datexs & Grating & $\mathrm{v}_{\mathrm{Na}}\left(\mathrm{km} \mathrm{s}^{-1}\right)$ & $\sigma_{\mathrm{Na}}\left(\mathrm{km} \mathrm{s}^{-1}\right)$ & $p E W_{\mathrm{Na}}(\AA)$ \\
\hline ASASSN-14hr & 2014-Oct-03 & $\mathrm{R} 3000$ & $-41.3 \pm 16.5$ & $76.3 \pm 13.6$ & $0.74 \pm 0.17$ \\
ASASSN-14jc & 2014-Oct-27 & $\mathrm{R} 3000$ & $-2.8 \pm 11.5$ & $65.3 \pm 10.6$ & $0.98 \pm 0.19$ \\
ASASSN-14jg & 2014-Nov-06 & $\mathrm{R} 3000$ & $8.9 \pm 15.3$ & $58.1 \pm 15.2$ & $0.23 \pm 0.07$ \\
ASASSN-15go & 2015-Apr-11 & $\mathrm{R} 3000$ & $82.9 \pm 12.1$ & $70.0 \pm 10.6$ & $1.75 \pm 0.32$ \\
iPTF13dge & 2013-Sep-19 & $\mathrm{R} 7000$ & $-35.1 \pm 3.3$ & $22.3 \pm 3.4$ & $0.34 \pm 0.05$ \\
LSQ14bcj & 2014-Apr-23 & $\mathrm{R} 7000$ & $20.9 \pm 4.7$ & $28.0 \pm 4.9$ & $0.99 \pm 0.19$ \\
PS1-14wl & 2014-Apr-02 & $\mathrm{R} 3000$ & $35.9 \pm 12.9$ & $70.0 \pm 0.0$ & $1.22 \pm 0.22$ \\
PSNJ13471211-2422171 & 2015-Feb-15 & $\mathrm{R} 3000$ & $14.5 \pm 49.6$ & $119.4 \pm 35.9$ & $0.71 \pm 0.26$ \\
PSNJ17194328-7721305 & 2013-Aug-30 & $\mathrm{R} 7000$ & $-70.7 \pm 3.2$ & $24.3 \pm 3.4$ & $0.47 \pm 0.07$ \\
SN 2012dn & 2012-Jul-21 & $\mathrm{R} 3000$ & $-9.4 \pm 19.8$ & $79.0 \pm 17.2$ & $0.25 \pm 0.07$ \\
SN 2013aj & 2013-Mar-08 & $\mathrm{R} 3000$ & $5.0 \pm 24.5$ & $95.2 \pm 18.8$ & $0.43 \pm 0.11$ \\
SN 2013cg & 2013-May-26 & $\mathrm{R} 7000$ & $42.0 \pm 23.2$ & $118.9 \pm 16.6$ & $1.39 \pm 0.25$ \\
SN 2013cy & 2013-Jun-03 & $\mathrm{R} 3000$ & $45.2 \pm 19.0$ & $42.5 \pm 20.8$ & $0.19 \pm 0.11$ \\
SN 2013er & 2013-Jul-31 & $\mathrm{R} 3000$ & $11.4 \pm 16.3$ & $78.4 \pm 13.3$ & $2.05 \pm 0.44$ \\
SN 2013gh & 2013-Aug-30 & $\mathrm{R} 7000$ & $-18.9 \pm 3.0$ & $23.3 \pm 3.1$ & $1.50 \pm 0.22$ \\
SN 2014aa & 2014-Mar-14 & $\mathrm{R} 3000$ & $-4.7 \pm 43.5$ & $130.0 \pm 0.0$ & $1.42 \pm 0.34$ \\
SN 2014ao & 2014-Apr-23 & $\mathrm{R} 7000$ & $-7.2 \pm 3.1$ & $23.7 \pm 3.2$ & $1.33 \pm 0.19$ \\
SN 2014at & 2014-Apr-23 & $\mathrm{R} 3000$ & $0.3 \pm 41.8$ & $130.0 \pm 0.0$ & $0.40 \pm 0.14$ \\
SN 2014bx & 2014-Jul-22 & $\mathrm{R} 3000$ & $55.4 \pm 12.9$ & $73.7 \pm 10.9$ & $4.34 \pm 0.79$ \\
\hline \hline
\end{tabular}

Table A5. Host galaxy redshifts for SN Ia Na sample.

\begin{tabular}{llll}
\hline \hline SN & \multicolumn{1}{c}{ Host } & Redshift & \\
\hline ASASSN-14hr & 2MASX J01504127-1431032 & 0.033620 & Ref. $^{a}$ \\
ASASSN-14jc & 2MASX J07353554-6246099 & 0.011320 & 6dF (Jones) \\
ASASSN-14jg & PGC 128348 & 0.015315 & Local \\
ASASSN-15go & (unknown) & 0.019030 & Local \\
iPTF13dge & NGC 1762 & 0.015854 & (Theureau et al. 1998b) \\
LSQ14bcj & MCG -02-36-015 & 0.036056 & Local \\
PS1-14wl & 2MASX J13324055-2036204 & 0.032346 & Local \\
PSNJ13471211-2422171 & ESO 509-G108 & 0.019910 & (Ogando et al. 2008) \\
PSNJ17194328-7721305 & ESO 044-G010 & 0.009587 & Local \\
SN 2012dn & ESO 462-G016 & 0.010187 & (Theureau et al. 1998b) \\
SN 2013aj & NGC 5339 & 0.009126 & (Theureau et al. 1998b) \\
SN 2013cg & NGC 2891 & 0.007952 & (Ogando et al. 2008) \\
SN 2013cy & ESO 532-G025 & 0.031465 & (da Costa et al. 1991) \\
SN 2013er & IC 850 & 0.018052 & (Grogin, Geller, \& Huchra 1998) \\
SN 2013gh & NGC 7183 & 0.009075 & Local \\
SN 2014aa & NGC 3861 & 0.016982 & (Cortese et al. 2008) \\
SN 2014ao & NGC 2615 & 0.014663 & Local \\
SN 2014at & NGC 7119 & 0.032242 & (Donzelli \& Pastoriza 2000) \\
SN 2014bx & NGC 6808 & 0.011570 & (Sandage \& Tammann 1981) \\
\hline \hline
\end{tabular}

${ }^{a}$ Via NED. 'Local' denotes local velocity measured with WiFeS, either from narrow lines in the SN spectrum or from galaxy rotation characteristics (see text for details).

a best fit relation of

$$
\Delta m_{15}=2.04 R_{\mathrm{Si}}+0.808 .
$$

The scatter about this best fit curve is 0.190 mag. 